Portland State University

PDXScholar

Summer 1-1-2012

\title{
Are We Cool Yet?: A Longitudinal Content Analysis of Nerd and Geek Representations in Popular Television
}

Christopher Louis Cardiel

Portland State University

Follow this and additional works at: https://pdxscholar.library.pdx.edu/open_access_etds

Part of the Broadcast and Video Studies Commons, Other Film and Media Studies Commons, and the Television Commons

Let us know how access to this document benefits you.

\section{Recommended Citation}

Cardiel, Christopher Louis, "Are We Cool Yet?: A Longitudinal Content Analysis of Nerd and Geek Representations in Popular Television" (2012). Dissertations and Theses. Paper 810.

https://doi.org/10.15760/etd.810

This Thesis is brought to you for free and open access. It has been accepted for inclusion in Dissertations and Theses by an authorized administrator of PDXScholar. Please contact us if we can make this document more accessible: pdxscholar@pdx.edu. 
Are We Cool Yet?: A Longitudinal Content Analysis of Nerd and Geek Representations in Popular Television

\author{
by \\ Christopher Louis Beauprey Cardiel
}

A thesis submitted in partial fulfillment of the requirements for the degree of

\author{
Master of Arts \\ in \\ Communication
}

\author{
Thesis Committee: \\ Leslie A. Rill, Chair \\ Priya Kapoor \\ Alma Trinidad
}

Portland State University

(C)2012 


\begin{abstract}
This study explores the representation of nerds and geeks in popular broadcast television programs over the course of the past twenty years. A content analysis of the five most popular scripted broadcast television programs for each year was conducted in order to assess the frequency of nerd characters, as well as the social competence, physical attractiveness, and demographic information of each such character. In addition, a supplemental survey design study was employed in order to collect public opinion data regarding perceptions of nerds in general and on television. The results of these studies indicated that while the per-year frequency of nerd portrayals has not varied significantly, nerds have been consistently portrayed as overwhelmingly white and male. Nerd characters in popular television programs have grown more physically attractive over the past twenty years. Furthermore, while technological or computer-related expertise remain significant predictors for the identification of television characters as nerds by audience members, the same is true for unattractiveness and low social competence. Considered through the theoretical framework provided by past mass media scholars, these findings suggest that nerds represent a group of individuals consistently portrayed as possessing technical aptitudes which are highly desirable in the current social context, but that such roles are portrayed as accessible only to white males.
\end{abstract}




\section{Dedication}

This thesis is dedicated to all the nerds, male, female and otherwise, without whom this project would not be possible. I am proud to count myself among your ranks. 


\section{Acknowledgements}

I would be remiss if I did not offer my most sincere and heartfelt thanks to:

Dr. Leslie Rill, without whose tireless guidance and insight this project would never have been accomplished, and who unceasingly exemplified a standard of scholarly excellence to which I will always aspire.

Dr. Priya Kapoor, for her wisdom and support throughout my graduate studies, and for the cultural awareness she helped me bring to my research.

Dr. Alma Trinidad, whose contagious passion for critical media studies is a true inspiration.

Jason Gauz and Sara Jackson, for their invaluable assistance in coding and establishing interrater reliability.

Ana Lapite, for her unending and unconditional belief in me throughout the course of my research. Few people would be willing to put up with being the partner of a graduate student, and I consider myself unimaginably fortunate to have found one of those few.

And my family, who have supported me without pause for the entirety of my life. I treasure every one of you, and nothing I accomplish would have been possible without the foundation you provided me. 


\section{Table of Contents}

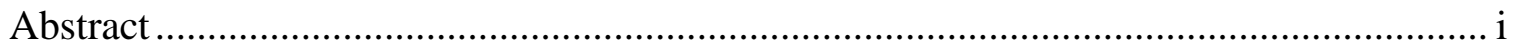

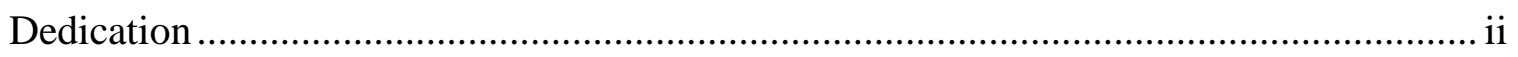

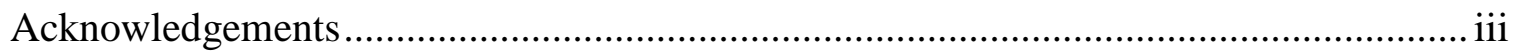

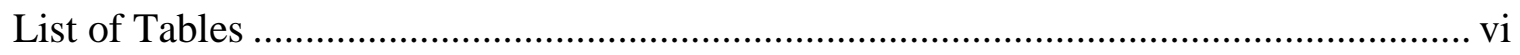

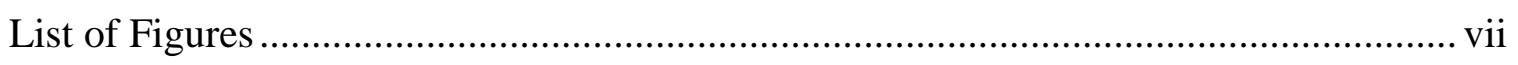

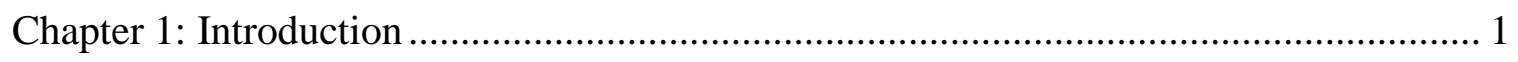

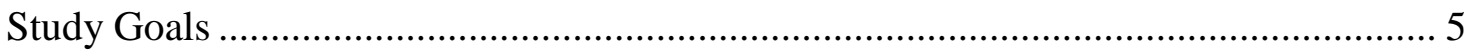

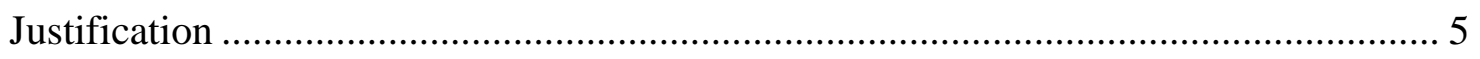

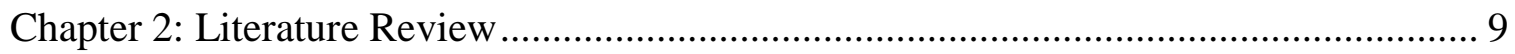

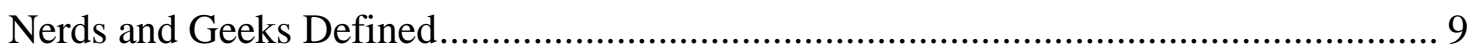

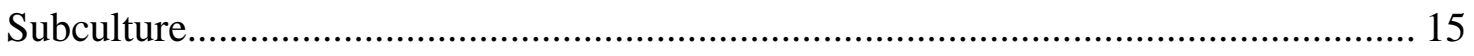

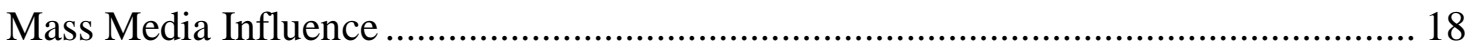

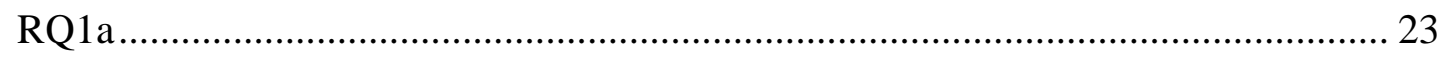

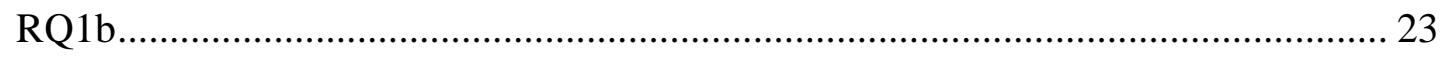

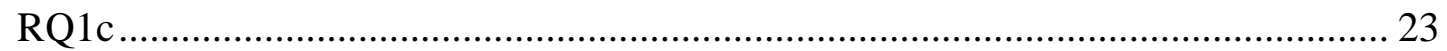

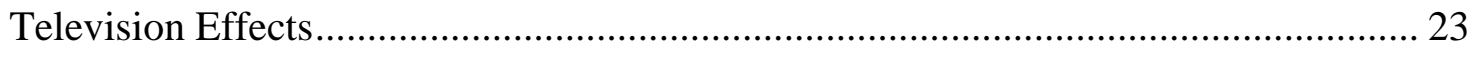

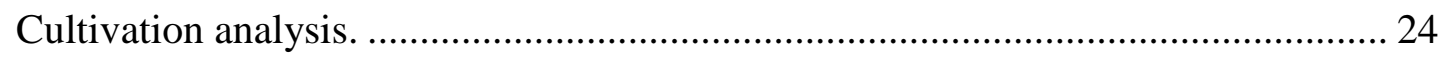

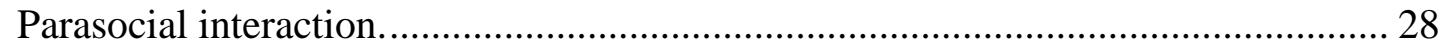

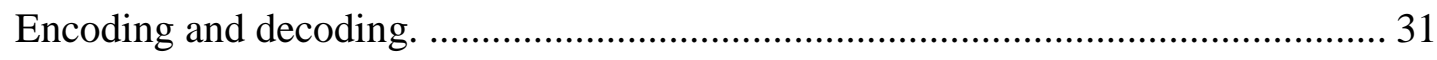

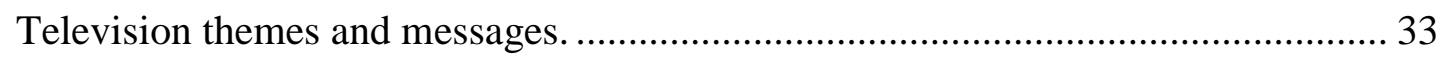

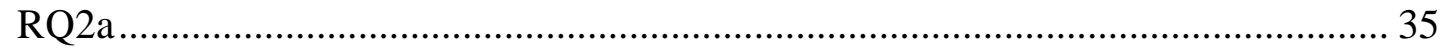

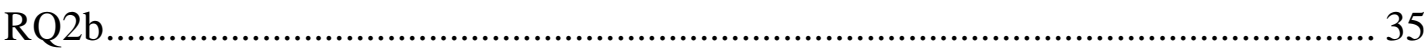

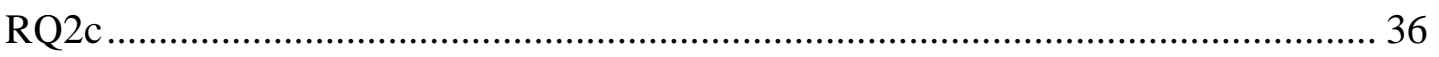

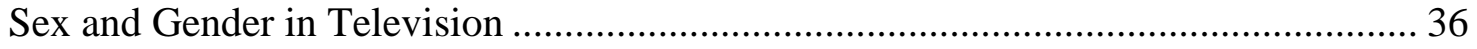

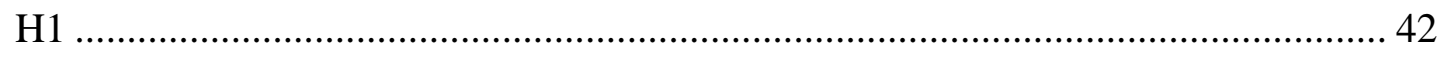

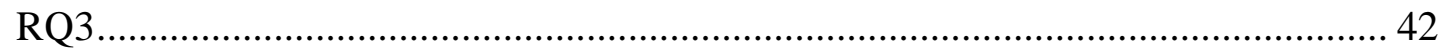

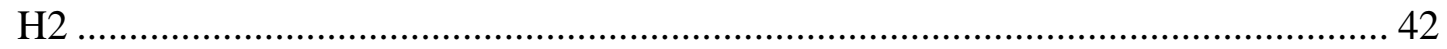

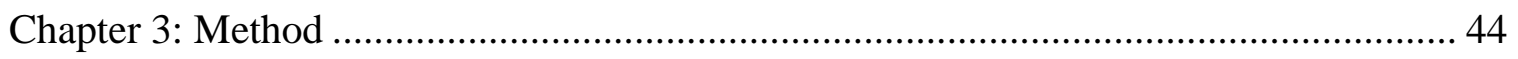

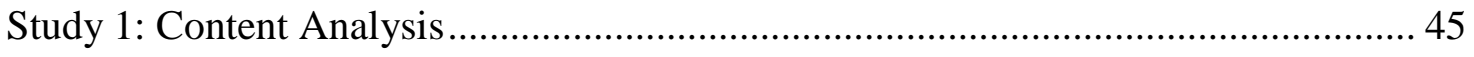


Sample

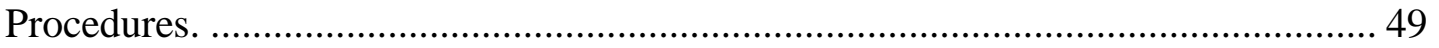

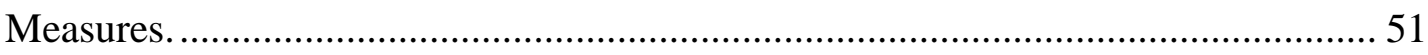

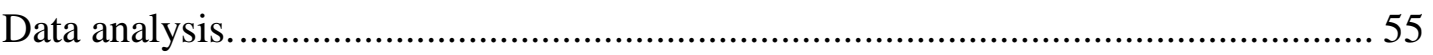

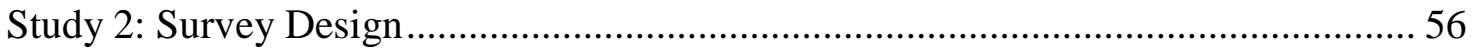

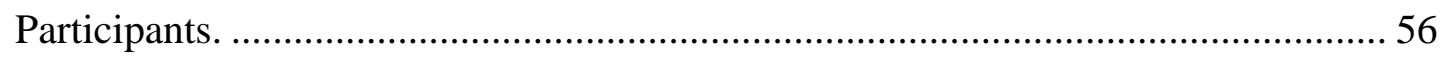

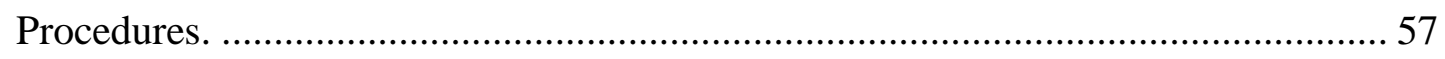

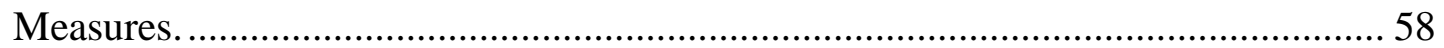

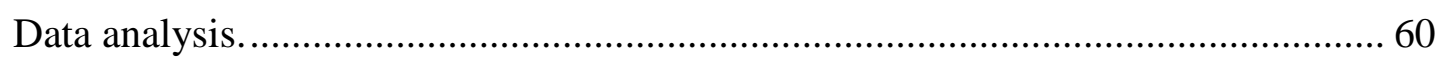

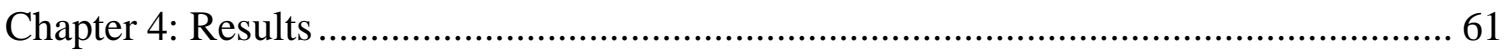

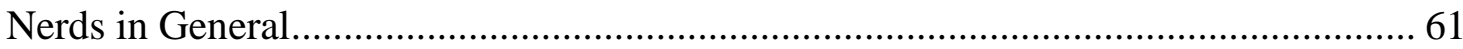

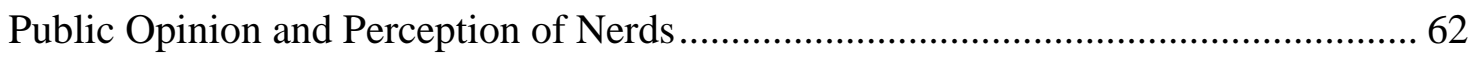

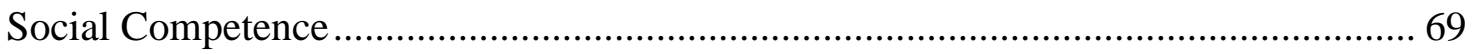

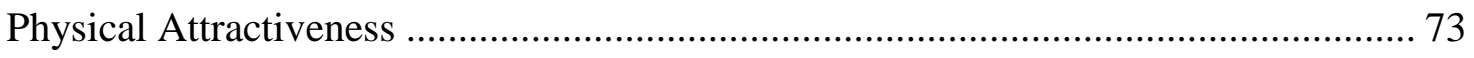

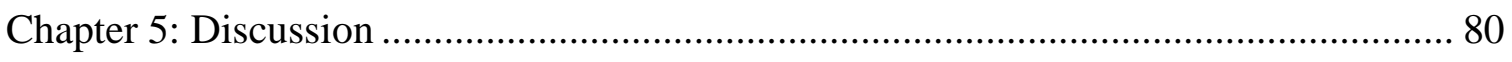

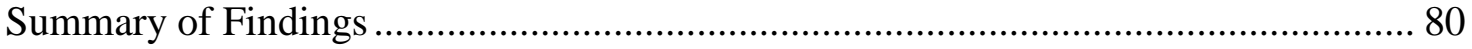

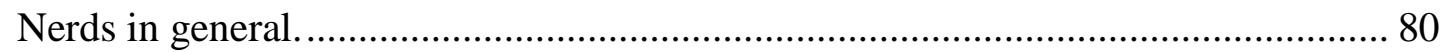

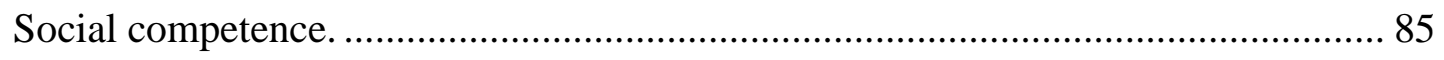

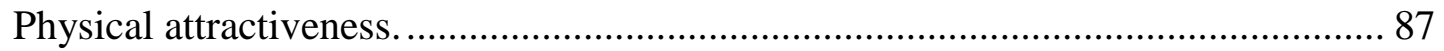

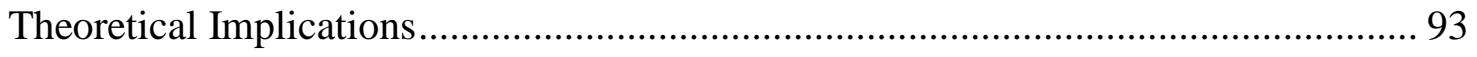

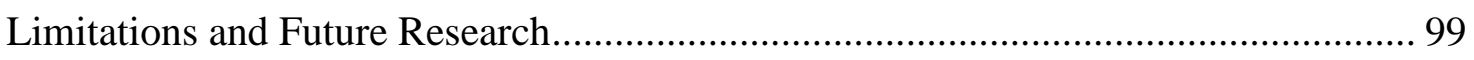

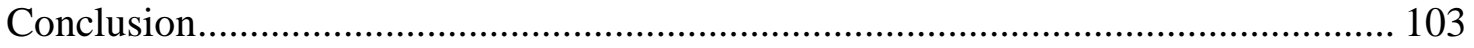

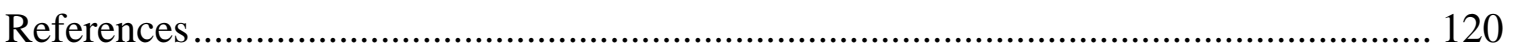

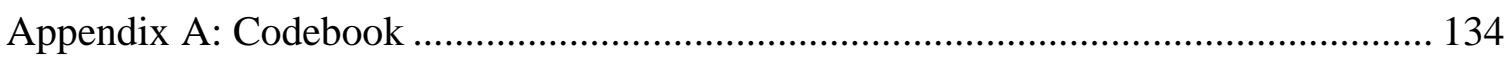

Appendix B: Survey Questionnaire ....................................................................... 146

Appendix C: Research Questions and Hypotheses ....................................................... 160 


\section{List of Tables}

Table 1: Most-Viewed Scripted Broadcast Television Shows by Year........................... 107

Table 2: Television Programs Included in Study Sample.............................................. 108

Table 3: Audience Perceptions of Popular Television Characters.................................. 109

Table 4: Assessment of Nerdiness of Popular Television Characters by Pro- and Anti-

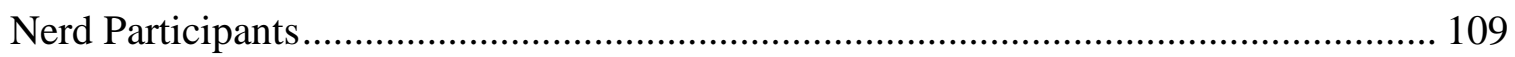

Table 5: Differences in Mean Assessments of Popular Television Characters on the Basis of Nerd Identification by Pro-Nerd Respondents ...................................................... 110

Table 6: Differences in Mean Assessments of Popular Television Characters on the Basis of Nerd Identification by Anti-Nerd Respondents ......................................................... 111

Table 7: Multiple Regression Analysis Summary for Predictors of Nerd Identification of

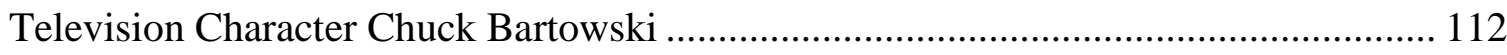

Table 8: Multiple Regression Analysis Summary for Predictors of Nerd Identification of Television Character Milhouse Van Houten................................................................ 113

Table 9: Multiple Regression Analysis Summary for Predictors of Nerd Identification of Television Character Dr. Sheldon Cooper ............................................................. 114

Table 10: Multiple Regression Analysis Summary for Predictors of Nerd Identification of Television Character Steve Urkel ............................................................................... 115

Table 11: Multiple Regression Analysis Summary for Predictors of Nerd Identification of Television Character Willow Rosenberg ................................................................ 116

Table 12: Assessment of Social Competence of Popular Television Characters by Proand Anti-Nerd Participants ......................................................................................... 117

Table 13: Assessment of Physical Attractiveness of Popular Television Characters by Pro- and Anti-Nerd Participants.................................................................................. 117

Table 14: Mean Assessment of Physical Attractiveness of Popular Television Characters

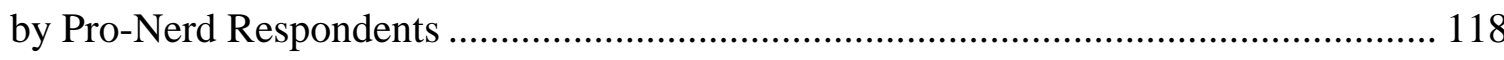

Table 15: Mean Assessment of Physical Attractiveness of Popular Television Characters by Anti-Nerd Respondents ....................................................................................... 118 


\section{List of Figures}

Figure 1: Frequencies of Nerd and Non-Nerd Characters By Year, 1992-2011 ........... 119

Figure 2: Frequencies of Nerd Characters in Comedic and Dramatic Programs By Year, 1992-2011 119 


\section{ARE WE COOL YET?}

\section{Chapter 1: Introduction}

In If I Ran the Zoo, a children's book written by the famous Dr. Seuss and published in 1950, the reader is taken on a fantastical (and often nonsensical) journey through an outlandish zoological menagerie. Nestled among the descriptions of such creatures as the Bustard, the Tizzle-Top-Tufted Mazurka, and the Preep, the young narrator of the story proudly proclaims that he will "bring back...a Nerkle, a Nerd, and a Seersucker too!” (p. 47). Just another handful of whimsical nonsense-creatures, seemingly no more remarkable than any other contained within the book's covers. And yet, within a year of its publication, the term "nerd" had entered cultural nomenclature in certain areas of the United States as a synonym for a "drip" or a "square" (Newsweek, p. $28)$; to be a nerd was equated with general undesirability and social, if not academic, underachievement. While the etymological progression of the term from this point forward remains widely debated (Burrows, 1998; Liberman, 2011; Zimmer, 2011), by the 1980s, nerds had entered the mainstream, as vividly evidenced by the 1984 release of the film Revenge of the Nerds.

In the film and its three sequels, the titular nerds are portrayed as physically frail, socially awkward, but intellectually and technologically proficient; the antithesis to the athletic and popular fraternity boys with whom they find themselves contending. If there had been any doubt of the recognizability of nerds before, all ambiguity in the term had now seemingly been eliminated, and the label became synonymous with the traits exhibited by the movie's main characters. Indeed, whereas early nerds were once considered in some sense underachievers, the current definition of "Nerd" includes 
ARE WE COOL YET?

"Underachiever" as a near-antonym to the term (Nerd, 2012). Perhaps equally

importantly, the subsequent twenty-five years has seen an increasing inclusion of nerds across all forms of mass media, from television to film and from magazines to books (Kendall, 1999). Part of this increase in popularity, within the last twenty years especially, is likely due to the explosion of high technology which has occurred during the same period, particularly in terms of the personal computer and other interactive technologies (The Digital Revolution, 1998). Whereas early nerds were portrayed in television and film as being proficient with technology in general, it became increasingly common during the first decade of the new millennium for such characters to appear in the role of the system hacker, the computer expert, or the software designer (Anderegg, 2007; Kendall, 2011).

Clearly, nerds reflected and continue to reflect a certain set of beliefs regarding technological ability and expertise, as well as the personal traits which accompany such expertise; how, then, have these beliefs become so firmly entrenched in American culture? Countless researchers (Gerbner, 1998; Gerbner \& Gross, 1976; Green \& Brock, 2000; McCombs \& Shaw, 1972; McLuhan \& Fiore, 1967; Smythe, 1954) have explored and discussed the influence of the mass media, and particularly of television, on the beliefs held by individuals and societies. It is generally accepted that mass media representations are a component, and an important one, in the process whereby opinions and perceptions are shaped. Lippmann (1922) famously describes the pictures in our heads, the images received through mass media channels which determine beliefs about individuals and groups with whom audience members might have no first-hand 


\section{ARE WE COOL YET?}

interaction. At some point, then, if a certain representation (such as that of nerds as being socially inept and technologically proficient) appears with sufficient frequency and constancy, this label will be applied by viewers of such representations as a statement of fact, an inarguable and essential demarcation of an individual's identity.

In addition to the concept of what it means to be a nerd, another term began to enter everyday parlance, this one with a distinctly different but equally unusual etymological background. The term "geek" was originally used in the American lexicon to refer to a circus performer whose act centered around biting the heads off of snakes, rats, and chickens (Geek, n.d.). However, by the middle of the $20^{\text {th }}$ century it had been transmuted into a term denoting familiarity with science or technology, and was employed as such by Robert Heinlein in the 1952 short story "The Year of the Jackpot." This usage has persisted throughout the past fifty years, with authors from both the popular press (Dooling, 2008; Pappademas, 2011; Robbins, 2011) and the academic community (Cross, 2005; Varma, 2000) embracing a definition nearly identical to that of the nerd. Interestingly, few if any films have provided the level of cultural recognition for geek as a label as Revenge of the Nerds has for the titular characters. However, television shows such as the short-lived comedy Freaks and Geeks, the still-running reality dating show Beauty and the Geek, and the independently-produced television series Pretty in Geek, which aired in mid-2011, help to ensure that the term remains within the common present-day vernacular.

In the case of both these terms, however, and in relation to their usage and representation in the mass media, certain patterns have arisen. Nearly without exception, 


\section{ARE WE COOL YET?}

nerds and geeks are portrayed as male; this, in conjunction with the increasing value of technological and computer-related occupations and skills, raises certain concerns.

Recent research indicates that women and men hold distinctly differing views on the values and pleasures of computer use (Stoilescu \& Egodawatte, 2010). Likewise, women continue to be severely underrepresented in undergraduate computer science programs compared to men, due almost entirely to a "computer culture" which both implicitly and explicitly privileges men (Margolis \& Fisher, 2002). While minimal literature exists to prove a causal relationship specifically between gendered characterizations in the mass media and choices of career or educational major, there is overwhelming evidence that the repeated and consistent portrayal of a given representation has an effect of some sort on audience members.

This, then, is the zeitgeist in which the current study is undertaken. While there remains some degree of contestation regarding potentially dissimilar meanings, after nearly a century of permutation and ambiguity, the definition of both nerds and geeks are largely aligned with individuals like those portrayed in Revenge of the Nerds. Such nerds and geeks, it seems, have begun to appear with increasing frequency in the mass media, although the positivity or negativity of these representations is debatable. Women remain underrepresented in computer science and related fields (Younker, 2011), a disparity which is mirrored in the gendered portrayals of nerds and geeks on television (Quail, 2011). This is perhaps unsurprising, as the effects of the mass media, and television in particular, have been well-documented, and appear to influence to some extent the beliefs and opinions held by audience members; further, these effects appear more pronounced 
ARE WE COOL YET?

with repeated exposure (Gerbner, 1998). With these facts in mind, the current study examined nerd and geek portrayals in popular television, between sexes and across the span of the past two decades.

\section{Study Goals}

In addressing the issues outlined above, this study has three proposed goals. First, the researcher hopes to provide a body of quantitative data regarding the trends of nerd and geek portrayals in the most popular scripted broadcast television programs of the past two decades in order to supplement the predominately qualitative and critical body of extant literature regarding gendered television portrayals. By contributing empirical quantitative data to the current corpus of academic knowledge, it is the goal of this study to provide a degree of generalizability which has heretofore been lacking. A second goal of the proposed study is to determine the extent to which nerd and geek portrayals on television have been gendered, and a third and final goal of this study explore not only the number of nerd and geek portrayals, but also the particular nuances and characteristics of those portrayals.

\section{Justification}

Although there are myriad reasons for which the undertaking of a study such as this might be considered beneficial, the three primary justifications are as follows. First, it seems that nerds and geeks have come increasingly into vogue in recent years, but in spite of this apparent trend, there has thus far been minimal scholarly attention devoted specifically to whether an increase in portrayals of nerds and geeks in popular television 


\section{ARE WE COOL YET?}

has in fact occurred. This dearth of previous research justifies the current study's inquiry into television portrayal of nerds and geeks, as well as its examination of the ways in which such portrayals have potentially metamorphosed over the course of the past two decades. This study examines the most popular scripted television programs from each year based on Nielsen ratings, a widely-accepted means of establishing viewing trends, in order to determine the extent to which nerds and geeks have, in fact, increased in prominence in recent years.

The popular press have, as previously stated, focused a good deal of coverage on the increasing visibility of nerds, both in regards to, for example, real life "alpha nerds" (Liberman, 2011) and in discussing television shows such as The Big Bang Theory (Jansen, 2011). It is possible, however, that this increase in popular press coverage is a reaction merely to a slight increase in the characterization, both on-screen and off, of certain types of individuals as nerds, rather than the overwhelming upwards trend in nerd and geek portrayals and self-identification which appears to be indicated. By undertaking a longitudinal study of nerd and geek portrayals, the question of the actual extent to which portrayals of nerds and geeks have changed, both in sheer frequency and in more specifically nuanced ways, can begin to be addressed in an empirical manner.

Second, gendered portrayals of nerds might potentially indicate deeply problematic trends in the perception of women's suitability for scientific or technological pursuits. The results of a study such as this could provide foundational research and evidence upon which future inquiry might be established, with the eventual goal of questioning and eventually effecting change in such perceptions and portrayals. In the 


\section{ARE WE COOL YET?}

course of this study, the frequency of nerd and geek portrayals is compared across sexes for each year included in the selected sample of television shows, and any disparities are then analyzed over the entirety of the two decades from which the sample of television shows was selected. If, as has been argued by many authors and scholars cited in this paper, a gender bias does exist in nerd and geek portrayals, this point must be addressed. As Blickenstaff (2005) notes, young women have difficulty finding themselves represented in science and technology careers both in textbooks and on television; this leads, he argues, to the perception on the part of these young women that these fields hold no place for them.

The aforementioned necessity of female representations of any sort in science and technology fields notwithstanding, if it is found that female nerds and geeks are overwhelmingly portrayed as more attractive, more competent, and more socially adept than their male counterparts, this could be considered a counterbalance of some sort to any observed numerical disparity. If, on the other hand, female portrayals are not only less common than male portrayals, but are also more negative in tone, the potentially problematic implications described by Blickenstaff (2005) might be amplified. As the cultural landscape of America continues to grow ever more wired and reliant on those with technological expertise, it is absolutely vital than an analysis such as this be undertaken to explore the ways in which nerds and geeks, the consummate "techies," are portrayed by the mass media.

Third, and perhaps most importantly, the power of television as a medium for the communication of ideas regarding the nature of the world, the individuals within it, and 
ARE WE COOL YET?

reality as a whole cannot be overstated. It must be emphasized that the messages contained in television programming are not merely static images with no lasting impact beyond the moment of audience perception. Rather, images and portrayals which occur repeatedly and over a prolonged period of time with little variation are capable of shaping the very way in which individuals view themselves and their surroundings. This phenomenon of creating social "scripts" (Meng, 2008; Wiederman, 2005; Wrench \& McCroskey, 2001) is not limited only to portrayals of nerds and geeks; whether we will it or not, television acts both as the backdrop to everyday interaction and as the surreptitious sculptor and interpreter of such interaction, and an exploration of television content in this case is thus unquestionably justified.

In the interest of ensuring that these goals are met, this study is grounded in extant academic and popular literature, a thorough examination and description of which is contained in the following literature review. This review synthesizes the works of scholars and authors who have previously explored the historical and current usage and representation of nerds and geeks, the effects and uses of the mass media as a whole and television in particular, and sex and gender in the media and in American society in general. Building upon the findings and conclusions contained within journals, newspapers, and books, a quantitative study is constructed which utilizes content analysis, in conjunction with a survey of public opinion regarding nerds and geeks, to achieve the goals outlined above. A full description of the methods employed in this study, including sampling and data collection and analysis techniques, is also included in a later chapter of this paper. 
ARE WE COOL YET?

\section{Chapter 2: Literature Review}

\section{Nerds and Geeks Defined}

While characters specifically crafted as "geeks" and "nerds" had been portrayed in film and television for nearly two decades prior, their increasing recognition and salience did not lead to study by the scientific community until later. Setting aside mass media conceptualizations such as those which have increasingly been integrated into American culture, it is beneficial to examine the ways in which the terms nerd and geek have been used and framed over the course of the past decade and a half within scholarly writing. While such an analysis of scholarly usage should not be considered sufficient in itself to provide a foundation for the current study, it provides a vital component of understanding when undertaken in conjunction with an examination of popular usage. In the pages that follow, the most common mass media conceptualizations and constructions of nerds and geeks will be explored, particularly those presented through the medium of television. Subsequent to this, such representations will be compared and contrasted with scholarly conceptualizations of nerds and geeks, in order to ascertain the extent to which popular and scholarly conceptions of these demographics conflict or align with one another.

In popular television shows such as Beauty and the Geek and movies following in the tradition of Revenge of the Nerds I through $I V$, nerds and geeks are portrayed almost without exception as highly intelligent but physically and socially awkward, unable to interact easily of effectively with those outside their own social circles, and in general 
ARE WE COOL YET?

constructed as the polar opposites of those individuals who are considered attractive and capable. The plot of the 1984 film Revenge of the Nerds centers around the rivalry between two prototypical nerds, both computer science majors, and the muscular, athletic, and popular members of a university fraternity. The titular nerds, Lewis Skolnick and Gilbert Lowe, are portrayed as physically frail and unassuming, with glasses perched prominently upon their noses, button-down business shirts serving in lieu of letterman jackets, and the pockets of those business shirts overstuffed with pens. In spite of these clearly illustrated and heavily emphasized physical shortcomings, however, Lewis and Gilbert manage to utilize their superior intellect and technological know-how to outwit and dominate their fraternity adversaries.

Arguably the first mainstream television character to personify (and, equally importantly, to popularize) these characteristics of the nerd was Steve Urkel, without whose overtly ridiculous antics the television show Family Matters would likely not have lasted beyond its first years (Haithman, 1991). Television programming in the past two decades especially is rife with similar portrayals of nerds and geeks as intellectually gifted but socially inept and often physically unappealing. From The Simpsons' Millhouse to the title character in the show Ugly Betty to nearly the entire cast of the popular Big Bang Theory, nerd and geek portrayals remain consistent. Almost without exception, characters which fit the description of nerds are characterized as antipodal to those other characters who, while perhaps less intelligent, simply "get" how to interact with other humans. It is interesting and perhaps unsurprising to note, then, that the preponderance of scholarly research dedicated to nerds and geeks relies heavily upon the 
ARE WE COOL YET?

physical and psychosocial generalizations employed by the mass media to inform its own inquiries.

Among the earliest scholars to begin analyzing geek and nerd identity and culture is Lori Kendall (1999), whose study of portrayals and images of nerds in United States popular culture has been in many ways groundbreaking. In the course of this article, Kendall draws upon the Oxford English Dictionary's definitions of a nerd, stating that these definitions "portray the nerd as an out-of-touch outcast, without necessarily associating nerds with intelligence or computers" (p. 262), a distinction which runs contrary to that most commonly adhered to by mass media characterizations. Kendall undertakes a critical content analysis of such popular press and mass media representations, pointing out that these representations tend to define nerds as "intelligent but socially inept and, beginning in the 1980s, as people overly involved with, and skilled in the use of, computers" (p. 262). In a later article, Kendall (2000) returns to the subject of nerds, noting in regards to an ethnographic study performed in an online community that the term carries varying connotations depending upon who speaks to whom and with what intent.

Through the remainder of Kendall's conceptualization of nerds, qualities are reiterated which align with the popularly constructed image of the nerd as an individual possessed of technological expertise, particularly as pertaining to computers. In Revenge of the Nerds, for example, Lewis and Gilbert must compete with their fraternity rivals in their university's Greek Games, and achieve victory through the creation and performance of a complex musical production made possible only through their 
ARE WE COOL YET?

familiarity and expertise with computers. Additionally, Kendall (1999) mentions that nerds are assumed to be male rather than female, as evidenced by the common modification of the term (to "nerdette" or "female nerd") to demarcate those instances in which this is not the case (p. 262). Kendall's conceptualization of nerds is closely echoed in Goldsborough's (2010) critical analysis, wherein the author draws on a number of sources before reaching the conclusion that nerds can be defined as "anyone with an above-average IQ and few gifts at small talk and ordinary social rituals” (p. 16). Numerous scholars (Cross, 2005; Goldsborough, 2010; Kendall, 1999) also include reference to a second term commonly conflated with nerd, that of the geek. The terms of nerd, geek, or both have been addressed by a multitude of other authors and researchers, and in nearly all cases the conceptualizations employed are similar enough that the terms might be considered interchangeable. As an example, Anderegg (2007) repeatedly refers to the "nerd/geek stereotype" (p. 4) of social awkwardness, physical unattractiveness, and exceptional intelligence without bothering to explicate any potentially problematic dissimilarities between the two terms included in this concept. Likewise, in Starcke's (2000) qualitative study exploring the evolution of nerd and geek identity among IT (information technology) professionals, no distinction is made between nerds and geeks.

As Cross (2005) states, the term of geek has multiple meanings; among the gifted students to which he devotes the majority of his study, for instance, it is a more pejorative version of "nerd," implying a still greater degree of social awkwardness. Among professionals and technophiles, however, it has begun to mean simply an individual whose passion for computers has permitted them to become experts in the field. The 
ARE WE COOL YET?

majority of scholarly and non-scholarly sources appear to be in agreement that the terms of nerd and geek are sufficiently similar to permit interchangeabilty. Smiler's (2006) content analysis of numerous identities and their intersection with masculinity enacted in a college environment reiterates the conceptualization of nerds as referring to "a physically weak, unattractive, poorly dressed male who favors academics and is not particularly engaged in the social scene" (p. 625). This definition is clearly very similar to that which is common in the mass media, and thus is highly relevant to the current study. In discussing gamer culture, Shaw (2010) employs a mixed-methods approach to the question of this culture aligns with cultural studies as a whole and notes in the process that "gamers, as geeks, are not expected to throw good parties" (p. 408), with no further attention devoted to the term of geek. Such a cursory mention implies that among some researchers, there remains the belief that nerds and geeks have certain inherent qualities which need not be elucidated for readers.

Distinctions such as those made by previous researchers (Goldsborough, 2010; Kendall, 1999; Varma, 2007), are worth noting. They are, however, sufficiently specific and nuanced as to be overshadowed by the far greater similarities between the concepts of nerd and geek, at least insofar as they will be applied in the current study, and it appears that four characteristics of nerds and geeks have consistently arisen. First, the majority of definitions constructed by prior researchers for both nerds and geeks include a higher-than-average level of aptitude for technology and the computer sciences. Second, many researchers note the implication of social awkwardness and unattractiveness which is concomitant with these terms. Thirds, both terms are more 
ARE WE COOL YET?

often employed as a negative assessment than as a positive one, although some individuals assign such labels to themselves and others as a mark of respect and admiration. Fourth and lastly, it appears that irrespective of positive or negative connotations, the term "nerd" implies a rejection of mainstream interests and priorities, favoring instead more intellectual, scientific, or technological pursuits.

It is possible to make three assertions regarding the concepts of nerds and geeks. First, they both "live" simultaneously within individuals, as identities which can be claimed or disclaimed, and within social interaction, as when certain sets of behaviors or traits designate an individual as a nerd or a geek, whether they will it or not. Second, while these terms are often considered distinct, they have been frequently conflated in a number of contexts due to the far greater similarities they share. Lastly, it is possible to assert that the characteristics which constitute nerds and geeks are generally considered self-evident, a perception likely exacerbated by the homogeneity of mass media representations.

In contrast to those scholars who make a distinction between nerds and geeks in that the latter are more socially adept than the former, the concepts should be understood to be interchangeable for the purposes of this study. Consequently, in the interest of parsimony, the term of nerd will be implemented in all subsequent discussion of these concepts for the remainder of this paper except where the term geek is specifically employed by a cited author. Building upon the foundation of the definitions provided by these scholars, my working definition of a nerd is an individual who possesses substantial knowledge and expertise in a specific field, particularly those related to computers and 


\section{ARE WE COOL YET?}

technology. Such individuals often have significant difficulty engaging in normal social interaction, as well as intimate interaction of a romantic or sexual nature. The term is gendered in that it is most often associated with males, and women who are assigned to the category are often seen as transgressing both social behavioral and gender norms. Similarly, nerds are nearly always portrayed as white and Caucasian rather than as members of any other racial or ethnic group; this is perhaps unsurprising, for with the possible exception of the "clown" or "entertainer" (p. 21), nerds do not fit cleanly into any of the categories Hall (1995) identifies as demarcated for non-white characters in popular television. In any case, just as it remains strongly associated with males as opposed to females or members of other sexes, the identity of the nerd currently tends to be constructed as a powerful signifier of whiteness.

It can also be claimed that nerds and geeks constitute a subculture, a group of individuals sharing certain norms, traits, and behaviors, but how has this subculture been constructed by both scholars and the mass media, and how is it presented to society? While the scholarly conceptualizations provided above are accurate in the main, attention must now be turned to the currently accepted usage of the terms of nerd and geek. It must be understood how these terms are created and portrayed, the role played by the mass media and scholars alike in communicating these portrayals, and how such communication shapes perceptions of the individuals to whom they are applied.

\section{Subculture}

An important facet of any analysis of nerds is the concept of subculture, the demarcation of a distinct group of individuals from the larger overarching group or 


\section{ARE WE COOL YET?}

culture. The concept of subculture is one which has been in use for several decades, and which has been at times hotly contested within academic circles in regards to its pertinence, salience, and suitability for describing the experiences of those it purports to encompass. While scholars (see, for example, Yinger, 1960) had already employed the term subculture for many years prior to the publication of Dick Hebdige's (1979) text Subculture: The Meaning of Style, it was this work which brought the field of subcultural studies to prominence. Hebdige's work was groundbreaking at the time, and remains the foundation upon which the current exploration of subculture in relation to nerds will be based.

Prior to the work of Hebidge, the term subculture had come into vogue in sociological and anthropological studies as shorthand for a wide range of arguably diverse and distinct concepts. In Rubington's (1958) critical article detailing a study of chronic drunkenness, the researcher appears to consider the term as essentially interchangeable with one's way of life. The definition employed by Patterson (1956) is more vague and amorphous, changing even throughout the course of the relatively brief article discussing a case study of adolescent subcultures. Arguing for a unification of this concept in order to permit more effective scholarly inquiry, Yinger (1960) states that in what was, at the time, current academic and scholarly work, the term subculture had been put to so wide a range of uses across so broad a spectrum of studies that its utility in any given case had been severely decreased. In contrast to the this attitude of free-wheeling interpretation exhibited by his contemporaries, Yinger urges the adoption of the term 
ARE WE COOL YET?

"contraculture" to distinguish those cultures which have as a distinguishing feature some aspect of conflict or direct contradiction with the dominant culture.

To apply Yinger's distinctions to the concept of nerds, if nerd culture could be said to operate independently of the larger culture without any of its rules or behavioral norms arising specifically in accordance with or in contrast to those of the latter, it could be considered a subculture. If, on the other hand, the ways in which nerd culture operates could only be understood through an analysis of the interactions between it and the culture without, Yinger would urge the use of the term of contraculture in lieu of that of subculture. Returning to the example of Revenge of the Nerds, while Lewis, Gilbert, and the other nerds in the film might be considered odd or unusual in any case, their identity as nerds is only fully evident within the context of the university, particularly when juxtaposed with the popular students who torment them.

It is at this point that Hebdige's (1979) text, in which the study of punk and other musical subcultures is described, gained prominence. Its clear articulation of the purpose to which the term is put, expressly vested in its contrast with the dominant culture (and thus, interestingly, aligned to some extent with Yinger's (1960) concept of contraculture), would influence the study of subcultures from that point forward. This articulation suits as well the current study, as nerds are understood to be nerds only through their deviance from mainstream culture. Without the backdrop of "normal" university students against which their social awkwardness and less-than-perfect physiques can be highlighted, Lewis and Gilbert would not be truly identifiable as nerds. 
ARE WE COOL YET?

Following on Hebdige's work, McArthur's (2009) textual analysis extends the findings of Hebdige's study into the realm of new technology and cyberspace.

Describing the formation and maintenance of geek subcultural groups, McArthur (2009) touches upon the general and overarching claim of this thesis: Namely, that mass media representations of nerds and geeks have been instrumental in delineating behaviors and traits by which such identities can be determined, ascribed, and avowed. Subcultures are created, shaped, and maintained not only through external forces, but through their interaction with the larger external culture. Among this array of forces at play in the cultural and subcultural dynamic, however, few are more powerful than the mass media, particularly in the current age of instantaneous access to information and entertainment. Given this dynamic, it seems sensible to follow this discussion of subculture with an examination of the ways in which mass media messages operate to reflect and, perhaps more frequently, create the reality by which individuals experience their worlds.

\section{Mass Media Influence}

In one of the earliest scholarly forays into the field of mass media and audience effects, Lippmann (1922) describes the nature of humans' relationship with reality as being not one of direct contact, but one which is mediated and shaped by, as he describes them, "the pictures in our heads." He cites a number of examples, historical and contemporary, of the ways in which the world is and has been viewed on an individual and cultural level - through these examples, he illustrates the ways in which the perceptions held by a given person do not merely (or even necessarily) reflect reality, but in fact shape their reality. The pictures which are carried within our heads are not, he 
ARE WE COOL YET?

claims mere images; rather, these pictures both reflect and shape the reality each person inhabits as their lives and their interactions with others are directed more by these pictures than by any sort of objective reality. To illustrate this point, consider again the general homogeneity of nerd representations discussed earlier; if Lewis and Gilbert of Revenge of the Nerds find themselves replicated in movies and television time and again, they become the referents from which individuals construct their mental picture of what nerds are.

Lippmann (1922) further explains that although he refers to these pictures as fictions, he does not conflate this term with that of lies. Rather, these pictures act as the immediate connection to a tangible reality which is necessarily lacking through the limited scope of human experience. In other words, while not everyone is likely to interact with a self-avowed nerd on a regular basis, mass media representations of nerds like Lewis and Gilbert provide us with a shorthand by which such individuals can appear to be known. This dynamic touches upon one of the most salient points made by Lippmann, and one to which he returns repeatedly: That these pictures in our heads constitute what he refers to as a pseudo-environment. A pseudo-environment, as Lippmann conceptualizes it, can be considered an individual environment comprised of fictions or semi-fabrications with which each person interacts, with the implications of these actions extending into the actual, external environment.

The pseudo-environment to which Lippmann refers is the environment, the reality, which surrounds each person and which is comprised of the pictures in their heads which lend meaning to the world around them. This pseudo-environment is said to 


\section{ARE WE COOL YET?}

operate between the individual and the external environment inasmuch as the actions, perceptions, and behaviors of the individual are in response to stimuli. These stimuli are filtered through the lens of the pseudo-environment but have implications in the external environment, that environment of which all individuals are a part. An individual who meets a young man similar in appearance and behavior to Gilbert or Lewis, who immediately assumes he is a nerd, and who treats him accordingly does so due to the influence of the pseudo-environment. Perhaps the young man in question is in fact entirely unfamiliar with mathematics or computers, perhaps he is exceedingly adept at social interaction, but none of this will matter, at least initially; the pictures in our heads have delineated a role for him, and it is that role to which he is assigned until he rids himself thereof. These pictures which comprise an individual's pseudo-environment are, therefore, anything but insubstantial in their implications for human behavior, interaction, and perception. Further, the pictures are to some degree mutable, and change can be affected by the replacement of one picture by another.

Further complicating the clear demarcation of media effects, Lippmann (1922) states that in regards to how an individual knows what she or he knows, particularly as pertaining to public personages, such knowledge is developed primarily through the personae which are displayed and the impressions and appearances which are made available on the public stage. Furthermore, the ways in which something is known are couched, necessarily, in terms which are manageable and familiar to the individual in whose head they reside. Think again of the example of Lewis and Gilbert; they, and the countless other representations of nerds which can be found in film and television, make 
ARE WE COOL YET?

it highly unlikely that, for instance, an attractive young woman will be considered a nerd, regardless of her computer expertise. This leads to the next point, the role played by media in shaping the pictures in our heads. In the last example, for instance, propaganda or other images presented on television, in popular press, and so forth might depict just such an individual struggle as a substitute for a more accurate representation of the battle being fought.

To bring this abstraction to bear on the concept of mass media representations of nerds, these portrayals, readily available in television programming, offer audience members an easily digestible shorthand version of individual human beings. Rather than being required to question the simplicity of such representations, audience members are provided a reference manual of sorts, by which they can identify who does and does not fall within these parameters. Just as Lewis and Gilbert of Revenge of the Nerds are recognizable to the other students at their university based on the characteristics they exhibit, those same characteristics are applied by viewers of the movie to inform and shape their own beliefs regarding who can (and must) be a nerd. It is because of this dynamic, emphasized in Golebiowska's (1996) experimental study, that Lippmann's (1922) arguments are foundational to the current study. Golebiowska's (1996) findings indicate that increased repetition of specific mass media images causes greater value and significance to be ascribed to those representations by audience members. Most important of all, the resultant beliefs have concrete and measurable effects on audience members' interactions with marginalized groups and individuals. As Hall (1995) argues, the mass media represent among the most powerful institutions of ideological production 
ARE WE COOL YET?

and determination present in society. Thus, Hall argues, media such as television do not merely reflect images devoid of implication; rather, they serve to both construct and perpetuate hegemonic conceptualizations of specific sociocultural groups.

Expanding upon this point, McCombs and Shaw (1972) articulate the theory of agenda-setting, describing the ways in which it operates, the role played by media sources in framing issues as important or unimportant, and the implications in terms of public perceptions and actions. At the core of agenda-setting theory is the concept that public opinion and attention does not necessarily direct mass media content, but rather, is directed by it. This concept can be stated most clearly and succinctly by stating, simply, that media matters; the images and representations disseminated through the mass media have an effect on consumers of said media. Whether an advertisement, a documentary, or simply a sitcom portrayal by which the beliefs of audience members are shaped regarding what exactly a nerd is, mass media messages have the inarguable potential to influence the realities of those who consume them. Numerous scholars have expanded upon and confirmed McCombs and Shaw's theory of the effects of agenda-setting in the fields of political policy and risk communication (Bakir, 2006; Hart, Nisbit, \& Shanahan, 2011; McComas, 2006), Environmental issues (Alm \& Davis, 1993; Melosi, 2000; Pralle, 2009), international relations (Kiousis \& Wu, 2008; Landolt, Goldring, \& Bernhard, 2011), and issues of race and gender (Bratton, Haynie, \& Reingold, 2007; Ertürk, 2004), among others. Across these fields and many others, the role played by the mass media in directing at least in part the attention of audience members is repeatedly illustrated. 


\section{ARE WE COOL YET?}

Clearly, then, McCombs and Shaw (1972) are not the only scholars to have engaged in discussion regarding the role played by the mass media in determining or influencing public perception. Xinsheng, Lindquist, and Vedlitz (2011) argue in their

quantitative study that the theory of agenda-setting explains in large part both public and political beliefs regarding global climate change; similarly, Tedesco (2005) employs agenda-setting as a framework for a quantitative content analysis of political discourse in the 2004 Presidential Primary. Among the central tenets of agenda-setting theory is the maxim, "The media don't tell us what to think; they tell us what to think about." Building upon the observations and findings of the authors cited in the preceding pages regarding the overwhelming importance and ubiquity of television messages, the following research questions are posed:

RQ1a: How have nerd portrayals in popular television changed in frequency in the past twenty years?

RQ1b: How have nerd portrayals in popular television changed in social competence in the past twenty years?

RQ1c: How have nerd portrayals in popular television changed in physical attractiveness in the past twenty years?

Through repetition of a specific set of messages, certain beliefs or subjects gain greater salience than those which are covered with less frequency; in addition to this dynamic, however, television effects theorists such as George Gerbner have argued that, indeed, television viewing does produce certain measurable effects among audience members.

\section{Television Effects}




\section{ARE WE COOL YET? \\ Cultivation analysis.}

Gerbner's (1998) work on cultivation analysis also touches upon the central argument that television provides programs to a very diverse and disparate audience, and that more importantly, the programming available through televised channels tends to have a homogenizing, "mainstreaming" effect on the beliefs and opinions of audience members. In regards to this last point, Gerbner clarifies that these effects are not immediate, but are only detectible over time. However, it is also stated that the effects will tend to increase over time, and therefore that by surveying television viewers and determining the length and frequency of viewing activity, a correlation should be found between greater amounts of viewing and increased acceptance of or adherence to mainstream opinions and ideals.

Gerbner (1998) begins his discussion of cultivation analysis by stating the claim that among all creatures, human beings are the only species to inhabit a world shaped by the stories they tell; this belief acts as the impetus for the cultural indicators and cultivation research projects which are described within his writing. Further explicating the theoretical framework within which the study of cultivation is constructed, Gerbner describes the ways in which storytelling, in one form or another, has become to a significant extent the jurisdiction of the mass media, and particularly of television. Again, the relevance to the current study is clear; as technological expertise becomes increasingly socially accepted, the frequency of portrayal of nerds in mass media rises accordingly, while these portrayals continue to both mirror the stereotypical nerd and urge a continued adherence to these stereotypes. So long as such stereotypes portray 
ARE WE COOL YET?

nerds as physically unattractive, as socially inept, and, perhaps most importantly, as male rather than female, it remains unlikely that audience members (particularly female viewers) will perceive nerd identities or occupations as desirable.

Building upon this understanding of the multiple influences which undergird every facet of television programming, it is worth noting the description of cultivation analysis provided by Gerbner (1998). Cultivation analysis refers in its simplest sense to the theory that increased television viewing will result in an increased acceptance or adherence to media-propagated conceptions of social reality. Thus, cultivation analysis explores the ways in which television exposure influences the development or maintenance of a specific outlook or system of beliefs, with this system of beliefs being traced back to the messages presented in the media. Gerbner makes explicit the fact that this influence is not unidirectional, but is dependent upon the group of viewers and their "position" relative to the mainstream messages which are televised. For example, in the context of the current study, audience members who have personally avowed a nerd identity and who take pride in the knowledge, skill, and expertise concomitant with such an identity might be less affected by the negative implications of television portrayals. This is not to say, however, that the effects of cultivation are mitigated entirely; such effects are lessened, perhaps, but it seems they cannot be completely erased.

The uses to which cultivation analysis has been put, however, extend beyond those of Gerbner's research. Morgan, Leggett, and Shanahan (1999) employ cultivation analysis in their quantitative examination of audience beliefs in relation to family portrayals on television. In addition to confirming their hypothesis that increased and 
ARE WE COOL YET?

prolonged exposure to specific types of televised messages increases audience members' avowal of associated beliefs, the authors further state that this approach has been implemented successfully in exploring beliefs regarding range of subjects which is wide indeed. Hammermeister, Brock, Winterstein, and Page (2005) explore the relative effects of moderate television viewing with an absolute avoidance of such viewing, using cultivation analysis as the theoretical framework to guide a survey-based study. Interestingly, the results of this study indicate that the effects of television cultivation are more pronounced among female viewers than among males.

Many have criticized Gerbner's (1998) work as being overly focused on violence and what has been dubbed "Mean World syndrome," the process by which heavy viewers of violent programming internalize the belief that televised violence accurately reflects reality. Images presented through similar portrayals and repeated across time are, according to cultivation analysis research, likely to lead to the increased acceptance and internalization of related beliefs and values among heavy television viewers. As an example of this concept, consider the fact that the same portrayals of nerds found in all four Revenge of the Nerds films have found a home in the popular television show The Big Bang Theory. Indeed, such television portrayals have become so ubiquitous that not only has the electronics store chain, Best Buy, dubbed their technical support personnel the Geek Squad, but this conceptualization of nerds has come full-circle in the television show Chuck's spoof of this group, the Nerd Herd. Given this, in concomitance with the role played by television in identity development and maintenance (Fisherkeller, 1997; 
ARE WE COOL YET?

Strelitz, 2003), the importance of mass media portrayals of nerds in shaping beliefs regarding their place in society should not be underestimated.

Gerbner (1998) also articulates the fact that it is inaccurate to say that television strictly reflects or creates reality. Rather, it is an integral part of a cycle of creation and maintenance which also incorporates the producers of messages and the individuals receiving those messages, with the viewing publics developing, to some extent, distinct identities based on this exposure. From this point, Gerbner further discusses the process of mainstreaming by which the perceptions and beliefs of individual audience members are gradually, over time and dependent upon the amount and frequency of television viewed, brought in line with those themes and representations which are most often presented. This effect is one possible exemplar of cultivation; it is also supported strongly by the findings of cultivation analysis, indicating that audience members incorporate the homogenizing messages and themes present in television programming into their own worldview.

Moreover, Gerbner's findings indicate that audience members need not be actively attending to television messages and images for cultivation to take place. Simple exposure, repeated often enough and across a sufficient period of time, will result in the gradual incorporation of commonly televised themes and beliefs into an audience member's worldview. If this is the case, it follows that television portrayals of nerds, complete with their depiction of traits and mannerisms which all nerds and only nerds exhibit, will have a definite and actionable effect on the perceptions and behaviors of audience members. Audience members are, it seems, increasingly likely to believe that 
ARE WE COOL YET?

men such as Lewis and Gilbert truly represent all nerds with every character they see on television who embodies this portrayal. This phenomenon illustrates, clearly and unequivocally, the need for a content analysis such as that undertaken here; if it is true that active participation in television message consumption is unnecessary for cultivation to occur, it is vital that we understand the types and frequencies of portrayals being presented in popular television.

\section{Parasocial interaction.}

This last point, that of viewer attendance and attachment to television characters, cannot be fully understood without undertaking an analysis of the phenomenon of parasocial interaction. In brief, this refers to the tendency of individuals to relate to celebrities or other people with whom they have no actual first-hand relationship as though they are, in fact, possessed of some degree of intimacy; in other words, to develop a relationship with a media personality which is, of necessity, non-reciprocal in nature. Particularly salient to the current study are the ways in which television audiences to relate to television characters, particularly fictional characters. In their study, Conway and Rubin (1991) employ a uses and gratifications framework to determine that a high degree of parasocial interaction is a strong predictor for the utilization of television for entertainment purposes. The effects of parasocial interaction have also been quantitatively studied in regards to children's selection of favorite television characters, with the findings indicating that although physical attractiveness predicts preference for both male and female characters, intelligence is only a predictor of preference for male characters. Whether this is due to a dearth of overtly intelligent female characters or a 
ARE WE COOL YET?

selective attendance only to attractive female characters is unclear, but it would appear that even on the rare occasions when female nerds appear on television, they stand a lesser chance of acceptance than do their male counterparts.

Having said this, however, studies have also been undertaken the results of which would appear to indicate potentially beneficial implications of parasocial interaction for increased acceptance and decreased stereotyping of nerds in television. Schiappa, Hewes, and Gregg (2006) employ a survey-based quantitative study design to ascertain the degree to which exposure to portrayals of gay men on the situation comedy Will and Grace correspond with levels of acceptance of gay men, with the results of the study indicating a strong causal relationship between frequent viewing and acceptance. This study supports the researchers' earlier findings which indicate that repeated exposure to gay male characters on the television shows Six Feet Under precipitated a significant decrease in levels of anti-gay prejudice (Schiappa, Gregg, \& Hewes, 2005). The results of the latter study did not, however, indicate any substantial changes in viewer attitudes after viewing the reality television show Queer Eye for the Straight Guy, which would appear to imply that reality television shows differ in some significant way from scripted programming.

This assessment is supported by Baruh's (2009) study, which employs both content analysis and survey design and the results of which indicate that the motivations for viewing reality television programming are much more strongly related to voyeurism than are the motivations for viewing scripted programming. In regards to program genre, the constraint of the current study to scripted broadcast television programming results in 


\section{ARE WE COOL YET?}

the including programs being constrained to one of two overarching genres, those of comedy and drama. The intricacies of the interplay between medium and genre are complex and multifarious (Askehave \& Nielsen, 2005; Mittell, 2001), with significant contention extant regarding the implications of newer visual media. However, it is contended that different genres of media texts, and the characters contained therein, serve to represent distinct and often contrasting ideologies in ways which are often designed to be readily digestible by audience members, particularly through the use of stereotypes and archetypes (Bednarek, 2011). It is hoped that the limitation of the current study to two broad genres of television programming, comedic and dramatic, will permit relatively straightforward analysis of resultant data; nonetheless, such analysis will assess any potential impacts of program genre on the portrayals of the nerd characters therein.

Given the roles played by comedic and dramatic television programs in the portrayal and perpetuation of various sociocultural and ideological beliefs (Bednarek, 2011), how, then, does parasocial interaction contribute to an understanding of nerd portrayals in television? As Annese (2004) argues in her qualitative study of mediated identity and parasocial interaction, individuals consistently monitor their behavior and their presentations of self depending upon the representations to which they are exposed in television programs. Thus, it stands to reason that if nerds are portrayed as highly intelligent but socially inept and physically unappealing, viewers will likely modify their own presentations in order to avoid the negative associations concomitant with such mannerisms. Viewers may assess their own behaviors, determine which of these behaviors could classify them as nerds, and attempt to henceforth avoid engaging in such 
ARE WE COOL YET?

behaviors. Additionally, the aforementioned findings of Conway and Rubin (2001)

would indicate that these effects are exacerbated for female viewers, as intelligent female characters do not enjoy the same levels of positive assessment as do intelligent male characters. Thus, female viewers who might otherwise feel drawn to computers and technology, and who might have the potential to enjoy and be highly productive in a technological career, are likely to downplay their fondness for such pursuits around others in order to avoid the negative connotations which they witness on television. Rather than engaging in pursuits which might, in fact, be of greater interest to them, female viewers who are exposed to persistently recurring portrayals of tech-savvy women and girls as social pariahs may well opt for adherence to more culturally accepted gender roles. It would be overly simplistic, however, to state that such a disavowal of apparently undesirable characteristics and behaviors will always occur; rather, a good deal of such decisions regarding behavior adoption or non-adoption is dependent upon the way in which a particular message is perceived, or decoded, by a particular viewer.

\section{Encoding and decoding.}

While Hall's (1973) work has been briefly mentioned above, it must be noted here that Hall emphasizes the fact that significant disparities can exist between the meanings which are encoded into a message based on a certain set of signs - a code - and the meanings which are decoded from that same message based on an altogether different code. Hall applies this model to the medium of television and the audience of messages disseminated thereby, stating that the messages produced in mass media are encoded with meanings by their producers, but may be decoded in a similar or divergent manner by 
ARE WE COOL YET?

viewers. Continuing from this thesis, Hall posits that unlike a physical stimulus such as a tap on the kneecap used to elicit a jerk of the leg, television messages do not act as a straightforward behavioral input with a given message causing a predetermined effect. Viewers watching Lewis and Gilbert being humiliated and abused in Revenge of the Nerds will not necessarily react with aversion; this reaction might be somewhat more likely than a desire to join the hapless young men in claiming a nerd identity, but a good deal depends on other factors.

Hall (1973) points out the complex nature of television signs, comprised as they are of visual and aural dimensions. Additionally, Hall discusses the fact that television signs are iconic in nature, as they are possessed, clearly, of some of the properties of the things they represent. What must be borne in mind, however, Hall argues, is that television signs cannot be seen to be the things they represent, as they are merely twodimensional signifiers of a three-dimensional world. However, this distinction is, in Hall's opinion, often missed. This explication brings Hall to the central point in relation to television messages, that messages which are intended (encoded) by producers and advertisers to hold one message may be perceived (decoded) by viewers in a slightly or altogether different manner.

Hall (1973) refers to the intended "mapping" (the encoding of meaning) on the part of producers in service of a hegemonic social order as the dominant or preferred meaning, and points out that individuals can, to some extent and in varying ways, resist this dominant meaning and decode or "read" messages in different ways (p. 135). This is readily evident in any study of the ways in which characterizations of nerds can be 
ARE WE COOL YET?

interpreted in differing ways by various audience members depending upon their alignment with nerd identity and culture. Again, one need only turn to Revenge of the Nerds for an example of this very concept: In spite of the stereotypical portrayal of nerds on the part of Lewis and Gilbert, and in spite of their mistreatment and humiliation at the hands of the more popular students, the film has become a cult classic and a favorite among real-life nerds of all stripes. Certainly, Hall's findings would support this idea, that ostensibly negative, stereotypically socially inept portrayals of nerds might be interpreted in a contrary manner. Likewise, however, it would appear possible that a portrayal developed as a progressive and positive response to the aforementioned stereotypical images could nonetheless be interpreted as a reiteration of these very stereotypes. Although the present study does not employ encoding and decoding as its primary theoretical frame, the theory is nonetheless of value in the present context due to the rationale it provides for the analysis of both television content and audience perception and opinion.

\section{Television themes and messages.}

Before moving on to the next point, it is beneficial to the purposes of the current study to engage in a final bit of explication of the concept of television effects, for as shall be seen through this final level of nuance, the degree to which the messages propagated through mass media channels can vary substantially. Smythe's (1954) conception of television reality is grounded in a more transactionist framework than is that espoused by Lippmann (1922). While the latter argues that television reality, and other forms of mass-produced images, serve strictly to create the pictures in our heads, 
ARE WE COOL YET?

Smythe (1954) would make the claim that an individual's beliefs and perceptions simultaneously shape the television messages which are received. Smythe (1954) engages in a micro-analysis of drama programming (the category which was found to occupy the greatest percentage of broadcast time during the years in which the sample was collected, 1951 and 1952), examining the roles portrayed and the types of individuals who were chosen to play those roles. Smythe further calculates the percentage of roles played by women and men, as well as the distribution of roles across racial categories and the degree to which these depictions align with reality or even random chance, and determines that the distribution of depictions corresponds closely with what were, at the time, "the values held by our culture" (p. 154).

Thus, it can be seen that Smythe's definition of television reality focuses to a much greater extent upon the ways in which that reality is co-negotiated, with both audience members and producers contributing their own layers of meaning and understanding. The message cannot be seen simply as a message, transmitting a clearly demarcated unit of information from sender to receiver; rather, there are additions made at both "ends" of this exchange which shape the reality which is intended and perceived. On a related point, Smythe makes the assertion that a television program becomes "more than the sum of its parts" (p. 143), a statement which is clearly in line with the definition of television reality described above. While the current study does not directly examine the degree to which this synergistic effect between message creator, message, and audience occurs, this study will attempt the first step - that of understanding the pictures being communicated. 


\section{ARE WE COOL YET?}

Smythe's (1954) viewpoint does align with Gerbner's (1998) concept of cultivation inasmuch as they both believe that television programming presents a representation of reality, and moreover, that these representations are never free of agenda or bias. While Smythe (1954) argues that such negotiation is an integral part of television viewing, and that television messages can be re-read and subverted depending upon the lens through which the audience views them, Gerbner (1998) focuses more upon the "mainstreaming" effects of television, whereby homogenization of opinion is maximized among viewers. Gerbner further states that any discussion of "which came first" between media messages and cultural assumptions is irrelevant, as all individuals are born into a cultural environment which they serve, in some way, to maintain.

The theories and studies outlined in the preceding pages have established that television programming affects audiences; bearing this in mind, it is recognized that the content analysis which serves as the primary design of the proposed study can be enhanced by simultaneously exploring public opinion regarding nerds, on television and off. Therefore, in order to ascertain the extent to which television portrayals of nerd characters aligns with public opinion of nerds, the following additional research questions are posed:

RQ2a: How are nerds in popular television currently perceived by viewers with positive or negative opinions regarding nerds?

RQ2b: What are audience perceptions regarding the social competence portrayed by nerd characters in current popular television programs? 


\section{ARE WE COOL YET?}

RQ2c: What are audience perceptions regarding the physical attractiveness portrayed by nerd characters in current popular television programs?

These cultural environments influence perceptions regarding essentially every aspect of human experience, from politics to soap operas, from nerds and geeks to the final subject of this literature review, the concepts of sex and gender.

\section{Sex and Gender in Television}

The concepts of sex and gender are ones which, while perhaps appearing peripheral to the study at hand, are in fact of significant importance thereto. The numerous media scholars cited in the preceding pages have argued that representations such as those disseminated through television are to some degree instrumental in shaping and maintaining audience beliefs about their world. Consider, then, that while Montemurro's (2003) content analysis of popular television shows reveals relatively few instances of sexual harassment employed as joke material in situation comedies, the programs included in the study included an average of over three jokes based on gender

harassment. The distribution of many types of roles is far from even between women and men, and the implications for the resultant disparities are potentially severe.

Many of the authors already cited have stated that nerds are more often than not conceived of as men, with female portrayals appearing more deviant from the mainstream even than their male counterparts. What are the implications, then, of this division and bias in such representation? If we accept, as Lakoff (1983) claims, that the technologization of discourse, academia, and society in general in accelerating ever more 


\section{ARE WE COOL YET?}

rapidly, the value of being able to claim a technologically-proficient identity becomes clear. And yet, as the results of Steinke's (2005) textual analysis of popular films reveal, even on the occasions when scientists, engineers, and other technologically-oriented professionals are portrayed as women, these women more often than not end up sacrificing their careers in order to "get the guy." Steinke argues that such representations act as powerful deterrents to young women and adolescent girls who might otherwise hope to enter professions such as those portrayed. It must be further emphasized that these are not mere suppositions on the part of the researchers; significant evidence exists to indicate that across nearly all scientific and technological fields of work and study, women have been and remain dramatically underrepresented (LaPonsie, 2012; Lee, 2002). This dynamic, however, begs the question of what underlying sociocultural forces are at play in determining this predisposition among the arbiters of mass media images; the answer, in short, is gender, and all the complications and assumptions attendant thereto.

In beginning this explication of the concept of gender, it should first be clarified that that this term is not, as is sometimes believed, interchangeable with that of biological sex. As Butler (1990) goes to great pains to explain, biological sex is that designation, most often "male" or "female," which can be (ostensibly) established by virtue of an individual's physical construction, particularly the sex organs. Gender, by contrast, is comprised of all the myriad and countless actions and behaviors which align an individual to a lesser or greater extent with "masculinity" or "femininity," both of which are socially constructed and maintained (Butler, 1990; Jackson, 1998). To draw once 
ARE WE COOL YET?

again upon Revenge of the Nerds, just as Lewis and Gilbert reify beliefs regarding what it means to be a nerd, the very fact that they and the majority of nerd representations to follow are male established a gendered bias within this identity.

To say that these genders are not biologically constructed should not, however, be perceived as detracting in any way from their very real and tangible effects on the lives of every individual living within the binary categorization system pursuant thereto.

Providing an example of this fact which is highly relevant to the current study, Olson and Douglas's (1997) mixed-method quantitative study on audience perceptions of gender representations on sitcoms reveals that gender roles in popular television have not progressed in equity as significantly as might be expected. While there are many who believe that gender is inextricably and inherently linked to an individual's biological sex, Butler (1990) argues that the two concepts are connected only inasmuch as such connections have been forged by a given society. There is, Butler claims, no inherent reason why any behavior, trait, or identity should be assigned to women or to men; rather, the gender norms and divisions which dictate everyday behavior are in existence in order to perpetuate male domination at the expense of women.

Gendered characterizations in television such as those posited to exist in portrayals of nerds are far from a new phenomenon, and a fair amount of research has previously been undertaken with the goal of understanding and explaining such trends. Quail's (2011) critical study, for instance, indicates that representations of nerds in television and film remain coded as white and male. Butler's (1990) work on gender not only crystallized a good deal of scholarly discourse on the concept in general, but also 


\section{ARE WE COOL YET?}

describes the ways in which gender is inscribed onto the body, not least by mass media messages. Representations of specific types of characters are created and portrayed in such a way as to make it appear that they are inherently the purview of one sex or another, serving to imbue these identities with a degree of gender normativity. For instance, just as Lewis and Gilbert portray the antithesis of coolness, the cheerleaders whom the titular nerds both lust after and fear are clearly demarcated as the epitome of femininity. The nerd, in the context of modernity, has been consistently portrayed as frail, unaggressive, and intellectually rather than physically gifted; all of these characteristics are antipodal to conventional masculine gender norms (outlined in depth by Kaufman, 1993). Indeed, in Revenge of the Nerds, Lewis succeeds in seducing the girlfriend of Stan, the President of the antagonistic fraternity, but this feat is only accomplished after Lewis disguises himself as Stan. Apparently, it seems, a nerd is only masculine and sexually attractive when he is not a nerd at all.

Historically, mass media representations have served to disseminate and reinforce the message that a woman's place was in the home, that women should strive primarily to attract men, and that only a handful of potential jobs were suitable for female occupation (Ceulemans \& Fauconnier, 1979). While such blatant messages have, to some extent, died away, Thornham (1998) argues that even today, women on the screen serve primarily as "spectacle" for men; Thornham echoes Hall (1973), however, in stating that contrary readings of televised representations are both possible and valuable. Thornham (1998) also reiterates the statements of many of the media scholars previously discussed in her assertion that, rather than simply reflecting reality, television and the other mass 


\section{ARE WE COOL YET?}

media in some sense create reality. This hopeful message is further strengthened by the findings of Losh's (2010) quantitative meta-analysis, which indicate that although men continue to outnumber women in technology- and science-oriented careers, parents are almost equally encouraging of daughters or sons entering such professions. By providing guidelines to both women and men for which behaviors, which traits, which identities are suitable and unsuitable for their sex, television, Thornham argues, acts as a powerful force in the maintenance of gender roles.

In addition to exploring the relative frequency with which nerds are portrayed as male or female in popular culture, it is necessary as well to examine the potential differences in these portrayals depending upon the sex of the character. Given the characteristics of nerds contained within the conceptual definition provided earlier, one variables which will be examined across gender lines is social competence, described by Rinaldi, Kates, and Welton (2008) as "the ability to achieve personal goals while maintaining positive relationships" (p. 129). Additionally, an abundance of research exists which supports assertions such as those indicated by White, Brown, and Ginsburg's (1999) content analysis of male and female body types on primetime television indicating that male characters are permitted a wider range of body types and are not required to be as physically attractive in order to establish a romantic attachment. Pursuant to these findings, the physical attractiveness of nerd characters will also be analyzed across sexes.

This, then, returns us to the central point of relevance between the concept of gender and the study at hand. If nerd identity is gendered as masculine, as Kendall 


\section{ARE WE COOL YET?}

(1999) states, and if Cross (2005) is correct in positing that those who are possessed of exceptional technological expertise and computational aptitude are considered nerds, it follows that women risk reprisal in displaying either of these traits. It is particularly interesting to note, given the bias toward male representations of nerds, that such male representations are perhaps more likely to persist across extended periods of time than are particular types of feminine representations.

Tsai (2010) argues that portrayals of women may have adapted more readily to changing sociocultural trends than have portrayals of men. Additionally, given the current study's focus on popular, mainstream television programming rather than more independent or fringe productions, certain properties of the former should be noted. As articulated in Miller's (2008) qualitative discussion of coverage of non-mainstream groups and movements, the mainstream media, and particularly television stations, tend to subtly delegitimize transgressive gender portrayals, often by simply opting not to provide them with coverage. This trend, however, may potentially be shifting, as indicated by a content analysis of television depictions of scientists based on gender performed by Long, Steinke, Applegate, Lapinski, Johnson, and Ghosh (2010).

Long et al. (2010) assigned gender characteristics to scientist-type characters depending upon their enactment of specific types of behaviors (e.g. caring and dependent for feminine, athletic and dominant for masculine), and examined these traits in conjunction with the biological sex of the character. The results of the study conducted by Long et al. would appear to indicate that while male scientists were represented more frequently than were female scientists, there was no statistically significant difference in 


\section{ARE WE COOL YET?}

gendered behavior exhibited by the characters examined. These findings are intriguing indeed, particularly given the potential similarities between characters portrayed as scientists and those portrayed as nerds. Based on these findings, the following hypothesis is posited:

H1: The frequency of portrayals of nerds in popular television programs will vary by sex, with male portrayals being more frequent than female portrayals.

In order to explore the relationship between the sex of nerds portrayed in popular television and those characters' levels of social competence, the following research question is posed:

RQ3: Does the level of social competence displayed by nerd characters in popular television programs vary by sex?

Lastly, bearing in mind the substantial body of extant literature which indicates that females on television are portrayed as physically and sexually desirable with greater regularity than are males (Duke, 2002; Field et al., 1999; Fredrickson et al., 1998; Grabe, Ward, \& Hyde, 2008; Groesz, Levine, \& Murnen, 2002; Holmstrom, 2004; Ward \& Harrison, 2004), the following final hypothesis is posited:

H2: The level of physical attractiveness displayed by nerd characters in popular television programs will vary by sex, with female characters being more physically attractive than male characters.

In concluding this review of the literature surrounding the concepts central to the current study, emphasis must once again be placed on the communicative effects of the 


\section{ARE WE COOL YET?}

mass media, and of television in particular. It can be argued that the portrayals of nerds in television programming constitute archetypes representative of extant sociocultural beliefs and norms regarding race, gender, and scientific and technological expertise (Guzman \& Stanton, 2009). This may well be; however, the existence of television nerds and archetypes of social mores and conventions does not preclude the possibility indeed, the likelihood - that such media portrayals simultaneously serve to perpetuate and legitimize the very cultural constructs from which they arise. The phenomenon of cultivation described by Gerbner (1998), McCombs and Shaw's (1972) explication of agenda-setting theory, and the parasocial interaction in which audience members engage with television characters as described by Conway and Rubin (1991), all underscore the overarching importance and influence of television in everyday life. Whether actively attended to or not, television messages are powerful communicators of ideas regarding what should be thought, why, and about whom. These messages may range from which toaster is most deserving of purchase to more problematic questions of gender and racial expectations. In every case, however, the degree to which such messages permeate individual perceptions and beliefs is extensive indeed, and are deserving of continued study by communication scholars. Through a longitudinal examination of popular television programming aired within two decades, in conjunction with a cross-sectional survey of public opinion, it is hoped that a more nuanced and complete picture of the ways in which nerds are represented and enacted might be crafted. The following chapter outlines the methodological processes and procedures employed in the course of the current study. 


\section{ARE WE COOL YET?}

\section{Chapter 3: Method}

In order to collect the data necessary for the exploration of the concepts and questions outlined previously, the current study employed a quantitative methodology. This study was conducted within a quantitative framework in order to alleviate to as great an extent as possible any biases or preconceptions harbored by the researcher (Babbie, 2004; Maxim, 1999), as well as to maximize generalizability of any significant findings (Babbie, 2004; Lund, 2005). Content analysis was the primary method employed; however, in order to ascertain current public opinion regarding nerds both in real life and on television, a supplementary dataset was collected through the implementation of survey design, distributed through Amazon.com's Mechanical Turk. The selection of this study design was predicated upon the necessity for both a means by which portrayals of nerds contemporarily and historically can be assessed and analyzed, and a measure of actual public perception of such portrayals. Although it was well understood that every methodological approach can provide valuable insights into the intricacies of television messages, due to the proposed study's focus on the changes in frequency and level of variables across time, it was believed that a quantitative framework is best-suited thereto.

Content analysis as a method permits the collection of first-hand data regarding television portrayals of nerds. Through the categorization of program content, content analysis is perfectly suited for an exploration of actual trends and themes within a body of extant texts (Krippendorff, 2004), in this case television programs. Additionally, the use of content analysis permitted the researcher to collect information pertaining to television programs which aired decades in the past without issues of recall or incomplete 
ARE WE COOL YET?

data. This is a strength which is not duplicable through any other method, and, given the necessity for longitudinal data in addressing the research questions and hypothesis previously outlined, solidly justified the methodological choice of content analysis.

In conjunction with an analysis of nerd portrayals in contemporary and recent popular television programs, this study necessitated the collection of data regarding public perception and opinion regarding nerds both on such programs and in reality. In order to address this need, a second dataset was compiled employing the method of survey design. By incorporating this survey-based dataset into the study design, the researcher had the ability to compare and contrast actual current perceptions of nerds with television trends and portrayals, leading to a far richer and more nuanced final analysis (see Babbie, 2004, for a detailed outline of survey design). Working within a quantitative methodological framework, survey design is unquestionably the method best-suited to ascertaining accurate information regarding general patterns and trends in opinion or perception across a large number of individuals. The following subsections outline these two studies, and include detailed descriptions of the procedures and specific methods involved in both.

\section{Study 1: Content Analysis}

\section{Sample.}

The units of analysis in the first study were artifacts; specifically, popular scripted American broadcast television programs were subject to analysis. The units of observation were the distinct character portrayals and representations contained within each episode. A total of 3,844 characters were observed during the course of this study; 
ARE WE COOL YET?

of these, 78 nerd characters were identified and included in data analysis. In addition to the 78 nerd characters coded in full, a total of 3,766 non-nerd characters were observed across the 300 episodes viewed; these characters were used for numerical comparative purposes only, and were subject to no further analysis. Of the 78 nerd characters observed, $89.7 \%(n=70)$ were male and $10.3 \%(n=8)$ were female. The racial breakdown was similarly uneven, with $92.3 \%(n=72)$ being identified as Caucasian, 2.6\% $(n=2)$ identified as Indian, and $1.3 \%(n=1)$ identified as African-American; the racial category of the remaining $3.8 \%(n=3)$ was unable to be determined in the course of coding. The units of analysis and observation were selected on the basis of their relevance to an examination of individual representation on television. The population for the study was all scripted American broadcast television programs; the sampling frame for the study was all episodes from the top five most popular scripted American broadcast television programming aired within the past twenty years, from 1992 through 2011. (This sampling frame did not include 2012, for which annual Nielsen reports had not yet been released.) Nielsen ratings were acquired through the Nielsen corporate website, Nielsen.com, by reviewing current and archival data, with the five most popular scripted broadcast television programs for each of the past twenty years being noted and compiled.

After collecting Nielsen viewing data on most popularly viewed television programs by year, Table 1 was constructed to represent the programs included in the study. As might be expected, numerous television programs appeared in the top-five lists during multiple years due to continued popularity among viewing audiences as assessed 


\section{ARE WE COOL YET?}

by the Nielsen rating company. Regardless, three episodes were selected from each year in which the program appeared in the top five scripted broadcast programs, in order to maintain a consistent analysis of static or mutable character representations across time within a given program. Rather than simply assessing whether or not certain characterizations are evident in popular programming, it was the goal of the researcher to determine the extent to which certain types of portrayals are present in the media, and in broadcast television programming specifically. The final sample included 300 episodes selected from a sampling frame of 37 unique television programs, with the number of instances in the top five ratings for each program ranging from 1 to $10(M=2.7, S D=$ 2.47). Table 2 provides a complete list of television programs included in the sampling frame, as well as the number of episodes selected for viewing from each program.

The research sample consists of these 300 total American television show episodes, selected through the utilization of a simple random sampling technique. No distinction was made between dramatic and comedic programming in terms of eligibility for inclusion; however, live-action programming such as sports broadcasts were not included in the sample. An analysis of reality television programming was likewise removed from the purview of this study. While Nielsen broadcast television ratings revealed a dramatic increase in the popularity of reality-based programs such as American Idol, Survivor, and Dancing with the Stars beginning in 2001 and continuing through the present day, there is evidence that significant distinctions exist between such ostensibly unscripted programming and the scripted television upon which this study was focused. Waggoner (2004) argues that the selectively edited nature of reality television 
ARE WE COOL YET?

programs concomitant with the belief held by many audience members that such programs are, in fact, reflective of reality cannot help but create distinct dissimilarities between reality and scripted programming. Bearing in mind the assertions of these researchers, as well as numerous others who have undertaken similar inquiries (BanetWeiser \& Portwood-Stacer, 2006; Gallagher, 2004; Moorti \& Ross, 2004) with results generally indicative of significant differences between scripted and "unscripted" television, reality television shows were excluded from the study at hand. Given the potential implications of such observed difference for the current study, it was decided that analyses of these program types should be undertaken separately in order to avoid any unwarranted conflation of effects.

The five most popular scripted broadcast television programs were selected from each year; this selection was made based on the belief that the greater a television program's popularity, the larger the audience, and consequentially the broader the potential effects of the messages within that program. From this sampling frame, three episodes were randomly selected from each television program's yearly broadcasts; it was believed that the selection of three episodes would provide the researcher with a sufficiently accurate picture of the characters who appear with some degree of regularity across the sampled television programs. This random selection was accomplished by assigning each episode a unique identification number. A table of random numbers was then generated, permitting the selection of a set of three episodes from each of the top five scripted broadcast television programs for each year. For example, each episode of Roseanne which aired in the year 1992 was assigned an identification number, with three 
ARE WE COOL YET?

of these numbers being selected at random and the corresponding episodes included in the study; this procedure was repeated with all episodes of Murphy Brown which aired in 1992, all episodes of Cheers which aired in 1992, and so for all of the 100 sampled yearly television programs.

\section{Procedures.}

Television programs were selected through an analysis of data obtained through the Nielsen Company, a national ratings service which monitors trends in the usage of mass media, including television. Sources such as DVD hard copies, paid-subscription viewing services such as Netflix, and free online services such as Sidereel were employed to provide access to popular television programs, both present and past. Specific episodes from each of the top five scripted broadcast television programs for each year were sorted into the study sample through the random selection process outlined above. Prior to the collection of the study's sample, a codebook (included in Appendix A) was created to facilitate the identification and analysis of the variables being evaluated. This codebook measured four content areas: characterization of nerds, social competence of nerd characters, physical attractiveness and body shape of nerd characters, and demographic information of nerd characters. These areas were coded primarily through an analysis of latent content such as reactions and subtextual cues, with the exception of those cases in which clear statements were made by characters in the programs which provide indication regarding the appropriate coding of one or more content areas. Nonnerd characters were coded only inasmuch as they are identified as such and subsequently 
ARE WE COOL YET?

removed from analysis; all nerd characters identified, regardless of their prominence within a given program, were coded in full in accordance with the coding procedures outlined in Appendix A.

Subsequent to the selection of the episodes to be included in the study sample, the researcher viewed each episode in full while coding for the presence of nerd characters. After this initial viewing, the research viewed the episode in full again and coded nerd characters for physical attractiveness and body shape, sex, age, race, socioeconomic status, and social competence (see Appendix A for full codebook). In addition, throughout the coding of the complete sample by the researcher, $10 \%$ of the total sampled episodes (30 episodes) were randomly selected to be viewed by both a second and a third coder, with results compared between coders in order to determine levels of interrater reliability as measured by Krippendorff's $\alpha$. Coders were trained in advance by the researcher in the specific traits, characteristics, and behaviors to which they should attend; coders also viewed a small sample of episodes not included in the study sample in order to become familiar with the coding process. If desired levels of interrater reliability (a minimum of .80) were not achieved for any of the episodes viewed by multiple coders, the coders and the researcher discussed areas of potential discrepancy and adjustments were made in coding schema in order to ensure that reliability could be assured. The episodes within the study sample to be viewed by multiple coders were selected randomly; interrater reliability was assessed through the use of Krippendorff's $\alpha$ for each of the study and demographic variables, with reliability ranging from .69 to 1.00 , as detailed below. 


\section{ARE WE COOL YET? \\ Measures.}

\section{Nerds.}

Nerds, the type of individual whose portrayal on television is of most central relevance to the current study, were conceptualized as follows: A nerd was considered to be an individual who possessed substantial knowledge and expertise in a specific field, particularly those related to computers and technology, and who often had significant difficulty engaging in normal social interaction, as well as intimate interaction of a romantic or sexual nature. The operationalization of nerds for the current study was achieved through the researcher's observation of individual characters in the programs included in the sample, with characters being coded for the presence or absence of nerd characterization based upon the following criteria. The researcher coded for 1) difficulties engaging in normal social interaction (specific behaviors include stuttering, being unable to speak, talking inappropriately, ignoring other individual(s), and appearing nonplussed by the behavior of others); 2) difficulties engaging in intimate romantic or sexual interaction (specific behaviors include stuttering, being unable to speak to a potential partner, appearing nonplussed by behavior of a potential partner, implication of poor sexual performance, and retreat from romantic or sexual interaction); and 3) identification as a nerd (or geek) by themselves or other characters. Characters identified as nerds by themselves or others were automatically considered nerds for the purposes of this study; when such identification was not made, characters were required to exhibit a minimum of three of the other characteristics outlined above (specific 
ARE WE COOL YET?

behaviors associated with difficulties engaging in normal and intimate interaction) in order to be considered nerds.

Nerd identity was considered a nominal variable, with characters identified either as "nerds" or "not nerds." Interrater reliability for this variable, as assessed through Krippendorff's $\alpha$, was .93. Krippendorff (2004) states that reliability levels greater than .80 are desirable, and recommends that conclusions be drawn only cautiously from variables with reliability levels between .67 and .80 . It has since been argued, however, that reliability levels between .90 and 1.00 are exceptionally high, levels from .80 to .90 are desirable, and levels from .70 to .80 should generally be considered acceptable, particularly given the stringent nature of Krippendorff's $\alpha$ (Lombard, Snyder-Duch, \& Bracken, 2010).

\section{Social competence.}

The variable of social competence was conceptualized as an individual's ability to interact with others in a manner generally considered appropriate and acceptable within the context of a given culture (Chen \& French, 2008; Koesten, 2004). Social competence was operationalized through the researcher's observation of each nerd character's interaction with other characters. The number of incidences per episode which elicited reactions of other characters indicating inappropriate behavior served as an additive index representing the variable as a ratio-level inverse measurement of social competence. Scores on this index for nerd characters included in the current study ranged from 0 to 8 , with a mean of 2.5 and a standard deviation of 1.67 ; bearing in mind that a score of 0 equates to no incidents of social incompetence, it appears that nerd characters in this 
ARE WE COOL YET?

study's sample tended to fall near the more socially competent end of this range.

Interrater reliability for this variable was acceptable at .71 as assessed though

Krippendorff's $\alpha$.

\section{Physical attractiveness.}

Similarly, physical attractiveness was conceptualized as the degree to which one's physical appearance and features are considered aesthetically or sexually appealing to others (Fan, Liu, Wu, \& Dai, 2004; Rohner \& Rasmussen, 2011). The variable of physical attractiveness was operationalized through the researcher's assessment of each character's physical appearance based on Neuendorf, Gore, Dalessandro, Janstova, and Snyder-Suhy's (2010) five-point measurement ranging from "Extremely unattractive" to "Extremely attractive." The attractiveness of a given character was determined in accordance with the criteria outlined by Schacht (2005), including facial and body symmetry, proportionality of facial features, and youthfulness of facial and body features $(M=2.79, S D=1.01)$. Interrater reliability for this variable, as assessed through Krippendorff's $\alpha$, was .69. While this alpha is lower than desired, it should be noted that it is significantly greater than that observed in Neuendorf et al's (2010) original implementation of this coding scheme, which achieved an interrater reliability level of .48 as assessed through Krippendorff's $\alpha$. Physical attractiveness is widely agreed to be highly subjective in nature and prone to fluctuation (Erwin, 1993; Lerner, Orlos, \& Knapp, 1976; Longo \& Ashmore, 1995); thus, lower alpha levels might be expected in regards to this variable. Additionally, characters were coded for the variable of body type, conceptualized as the shape and size of an individual's physical form and 
ARE WE COOL YET?

operationalized through the implementation of Thompson and Gray's (1995) nine-point scheme $(M=4.86, S D=1.74)$, with an acceptable Krippendorff's $\alpha$ level of .76.

\section{Demographic and program variables.}

Nerd characters were also coded for demographic variables including sex, estimated age group, socioeconomic status, and race. Sex was operationalized by observing each nerd character's physical appearance and the pronouns used in reference to that character (Krippendorff's $\alpha=1.00$, indicating perfect consistency between coders). Estimated age group was operationalized based primarily upon manifest physical attributes (hair color, wrinkles, etc.) as well as activities and lifestyle (grade school student, college student, parent, etc.). Of the 78 characters identified as nerds, $55.1 \%(n=43)$ were coded as "Young Adult," 35.9\% $(n=28)$ were coded as "MiddleAged," 5.1\% $(n=4)$ were coded as "Child/Pre-Teen," 2.6\% $(n=2)$ were coded as “Teenager," and 1.3\% $(n=1)$ was coded as "Elderly." (Again, coders achieved a perfect Krippendorff's $\alpha$ level of 1.00.) Socioeconomic status was operationalized by observing the conditions in which that character lives, their possessions, their state and manner of dress, and the extent to which they discussed money as a cause for concern in their lives. Of the 78 nerd characters included in this study, 59\% $(n=46)$ were coded as "Exhibits no clear financial need or exceptional affluence," 7.7\% $(n=6)$ were coded as "Exhibits exceptional affluence," 6.4\% $(n=5)$ were coded as "Exhibits clear financial need," and 26.9\% ( $n=21)$ were coded as "Unable to determine." (Krippendorff's $\alpha$ for this measure $=77$, indicating an acceptable level of reliability.) 


\section{ARE WE COOL YET?}

Race was operationalized, whenever possible, by observing statements made by that character or by others in reference to that character. In cases in which such statements were unavailable, coders determined race based upon skin tone and physical features, when such features were sufficiently unambiguous as to provide a reliable coding. Of the 78 characters coded as nerds, $92.3 \%(n=72)$ were Caucasian, $2.6 \%(n=$ 2) were Indian, and $1.3 \%(n=1)$ were Black, with the remaining $3.8 \%(n=3)$ being coded as "Unspecified/Unable to determine." (Krippendorff's $\alpha=1.00$, indicating perfect interrater reliability.) Lastly, the genre of television program from which each nerd character was drawn was operationalized through attention to the general tone and structure of each episode viewed, with particular weight given to aural cues (such as the inclusion of laugh tracks or intense or ominous music). Programs were coded as either comedic or dramatic in nature; of the 78 nerd characters identified, $32.1 \%(n=25)$ were observed in dramatic programs, while $77.9 \%(n=53)$ were observed in comedic programs. (Krippendorff's $\alpha$ for this variable $=1.00$, with no discrepancies arising between coders.)

\section{Data analysis.}

Statistical analyses were conducted through the utilization of IBM SPSS Version 19, with a focus on determining longitudinal television trends regarding frequency, social competence, and physical attractiveness of nerd portrayals, as well as differences in means in physical attractiveness and social competence between male and female portrayals of nerds and frequency of male and female portrayals. Chi-square analysis and 
ARE WE COOL YET?

one-way ANOVAs were employed in the assessment of the former points, while chisquare analysis and independent-samples $t$ tests addressed the latter.

\section{Study 2: Survey Design}

\section{Participants.}

The research participants consisted of 388 individuals whose questionnaire submissions were collected through Amazon.com's Mechanical Turk. Anonymous individual contributors who were registered as "Workers" (the term used by Amazon in reference to those individuals who complete surveys such as the one employed for this study) on Amazon.com's Mechanical Turk site were subject to analysis. Of the individuals included in this sample, 54.4\% $(n=211)$ were male, while $45.1 \%(n=175)$ were female and $.6 \%(n=2)$ reported as "Other" or did not disclose their sex. The study sample was $39.9 \%$ Indian $(n=155), 28.6 \%$ Caucasian $(n=111), 20.4 \%$ Asian/Pacific Islander $(n=79), 3.9 \%$ African-American/Black $(n=15), 2.3 \%$ American Indian/Alaskan Native $(n=9)$, and $2.1 \%$ Hispanic or Latino/a $(n=8)$, with the remaining $2.8 \%(n=11)$ identifying as "Two or more races," "Other," or not disclosing their race. Participants ranged in age from 18 to 67, with a mean age of the sample being 29.4 and a standard deviation of 9.15. The questionnaire was distributed through Mechanical Turk, an online survey-distribution tool which, although technically a volunteer-based sampling technique, has been determined to provide high-quality generalizable data at least as reliably as traditional random sampling techniques (Buhrmester, Kwang, \& Gosling, 2011). Questionnaire items include questions measuring perceptions of nerds in general as well as of five specific characters from current and recent popular television programs. 
ARE WE COOL YET?

Although this study employs a sampling technique which could ostensibly be considered a voluntary method, and thus nonprobability and nongeneralizable, as stated earlier, previous research has established that the quality of Mechanical Turk data is at least equal to that available through most random sampling methods. From this sampling frame, 388 questionnaires were collected, at which time data collection ceased and the survey on Mechanical Turk was made unavailable to users.

\section{Procedures.}

The researcher collected data regarding public opinion and perception of nerds, both in television programming and in reality, employing survey design as a method. First, a questionnaire (Appendix B) was developed which collected demographic and media use information from participants, as well as information pertaining to participant opinion and perception regarding nerds in general. The questionnaire also included questions regarding specific television characters generally perceived as nerds, as indicated by an analysis of online articles regarding popular nerds on television (Bricken \& Heiler, 2009; Chruscinski, 2008; Stice, 2011). This survey-design study was predicated upon the argument that television portrayals of nerds are related to audience beliefs, and that it would therefore be something of an oversight to neglect to establish the degree to which these portrayals were, in fact, perceived as nerds by viewers. All data collected through the implementation of this questionnaire was, however, intended primarily to supplement and validate the primary content analysis portion of this study.

Prior to collection of survey data, IRB approval was acquired for the inclusion of human subjects in this study. Once IRB approval was acquired, the questionnaire 


\section{ARE WE COOL YET?}

outlined above was made available to participants on Amazon.com's Mechanical Turk, with each participant receiving reimbursement of $\$ .25$ upon satisfactory completion of the questionnaire. This reimbursement amount, while seemingly small, was assessed as sufficient to obtain approximately four hundred accepted responses in less than three days (Buhrmester, Kwang, \& Gosling, 2011); the average completion time for this questionnaire was 11 minutes and 31 seconds. After the requisite number of questionnaires was collected, the survey was closed on Amazon.com's Mechanical Turk, at which time no further participants were accepted.

\section{Measures.}

Public opinion and perception of nerds were measured through the implementation of a 100-item questionnaire (Appendix B) The survey began with an open-ended question asking participants what they believed to be the top five characteristics of a nerd. The first questionnaire section after this included 18 five-point Likert-style questions, beginning with five measuring individuals' general perceptions of nerds; these included items such as "I think of myself as a nerd" and "I think being considered a nerd is a good thing (Cronbach's $\alpha=.88$ ). The next three questions measured perceptions of nerds' intelligence, technical aptitude, and engagingness through items such as "Most nerds know a lot about computers, science, or both." Following this, the questionnaire included two items assessing perceptions of nerds' social competence, with these items being "Most nerds don't know how to act around other people" and "Most nerds are uncomfortable with or confused by romance" (Cronbach's $\alpha=.65$ ). Subsequent to this, the questionnaire included three items tapping into perceptions of 
ARE WE COOL YET?

nerds' physical attractiveness; due to low alpha levels, only the item with the highest loading value, "I could imagine being physically attracted to a nerd," was employed in the assessment of this variable. These questions aligned with the researcher's conceptualization of nerds as individuals who possess substantial knowledge and expertise in a specific field, particularly those related to computers and technology, and who are often perceived as physically unattractive and having significant difficulty engaging in normal social or intimate interaction. The final question in this section measured perceptions of nerds as one of three responses: male, female, or not predominately one more than the other.

The second section of the questionnaire was divided into five sub-sections, each discussing a specific television character generally considered a nerd. Within each of these sub-sections, participants were asked one initial yes-or-no question, “Are you familiar with this character?", followed by six questions employing five-point Likertscale response categories regarding perceptions and opinions of the character and eight seven-point questions drawn from McCroskey, Richmond, and Daly's (1975) Perceived Homophily Measure. (Cronbach's $\alpha$ for this scale $=.81$ for Chuck Bartowski, .85 for Milhouse van Houten, .83 for Sheldon Cooper, .80 for Steve Urkel, and .81 for Willow Rosenberg.) This second questionnaire section was designed to ascertain the extent to which certain television characters, which were selected on the basis of their repeated inclusion in lists and articles describing "Television's greatest nerds" (e.g., Bricken \& Heiler, 2009; Chruscinski, 2008; Stice, 2011), were in fact perceived as such by participants, as well as the extent to which viewers identified themselves as similar to 
ARE WE COOL YET?

each character. Images of each character were included, and responses were analyzed controlling for preexisting familiarity with the characters. The third and final section included four participant demographic questions and six media use questions; these items were used as control and grouping variables in the analysis of public opinion and perception.

\section{Data analysis.}

Statistical analyses were conducted through the utilization of IBM SPSS Version 19, with a focus on determining cross-sectional public opinion regarding nerds in television and real life. Independent samples $t$ tests, Pearson product moment correlation, and standard multiple regression analyses were utilized to explore general perceptions of nerds, as well as perceptions of physical attractiveness and social competence of nerds in television and in general, with participant demographic and media use questions employed as control and grouping variables. 


\section{ARE WE COOL YET?}

\section{Chapter 4: Results}

The following statistical analyses are organized by theoretical construct rather than in the originally established order of research questions and hypotheses. It is hoped that this organizational system will facilitate maximum parsimony and clarity of results.

\section{Nerds in General}

In order to assess the degree to which the frequency of nerd portrayals in popular broadcast television programs has changed over the course of the past twenty years (RQ1a), a Pearson chi-square analysis was conducted. First, an independent samples $t$ test was performed which established a statistically significant different between numbers of nerd $(M=3.90, S D=3.53)$ and non-nerd $(M=188.45, S D=27.89)$ portrayals by year, $t(38)=-29.35, p<.001$. After this difference in means was established, the frequency of nerd portrayals was crosstabulated with the frequency of non-nerd portrayals by year; this analysis revealed no statistically significant changes in these frequencies over time, $\chi^{2}$ $(126, N=20)=134.16, p=.29$. As illustrated in Figure 1, portrayals of nerds in popular broadcast television programs appear to have remained relatively constant in their frequency relative to portrayals of non-nerd characters. As a post hoc measure intended to permit more nuanced statistical analysis during subsequent tests, an additional Pearson chi-square analysis was conducted in order to assess frequency of nerd portrayals in comedic and dramatic television programs by year. This analysis revealed no statistically significant changes in these frequencies over time, $\chi^{2}(35, N=20)=25.50, p=.88$, indicating that the number of nerd characters present in popular television programs has remained relatively consistent over the past twenty years. As illustrated in Figure 2, 
ARE WE COOL YET?

however, there were distinct periods of time during which nerd characters appeared primarily in comedic or dramatic programs. In light of the lack of variance in frequency of nerd portrayals as a whole, where noted below, sampled years were grouped by fours (1992 to 1995,1996 to 1999,2000 to 2003,2004 to 2007 , and 2008 to 2011) in subsequent analyses in order to facilitate maximally parsimonious explication of results.

While the frequency of nerd portrayals as a whole has remained consistent, the researcher posited that male nerd characters will appear more frequently than will female nerd characters (H1). In order to test this hypothesis, an independent sample $t$ test was employed to test the hypothesis that the number of nerd portrayals in popular television programs per year will vary by character sex, with male portrayals being more common than female portrayals. This analysis revealed that the average number of male nerd characters per year $(M=3.50, S D=.2 .87)$ differed significantly from the average number of female nerd characters $(M=.40, S D=.82)$ as predicted, $t(38)=4.63, p<.001$. Nerds in popular broadcast television programs were disproportionately (and overwhelmingly) more likely to be male than to be female; thus, hypothesis one was supported. Data collected through this study's survey component, intended to collect supplemental public opinion data regarding nerds, provided further support for the association of nerdiness with males. Responding to a questionnaire item assessing perceptions regarding the usual sex of nerds in general, 56.2\% $(n=218)$ of participants stated that nerds were more likely to be men, while $34.8 \%(n=135)$ stated that there was no difference and only $8.8 \%$ $(n=34)$ perceived nerds as predominately female.

\section{Public Opinion and Perception of Nerds}




\section{ARE WE COOL YET?}

In preparing to explore the ways in which individuals currently perceive nerds in popular television (RQ2a), participants' levels of positivity toward nerds were first assessed using a five-item scale comprised of five-point questions, with lower scores indicating more negative perceptions. This assessment revealed a mean level of 2.81; participants were subsequently divided into two groups, with those scoring above this point considered "pro-nerd" and those below considered "anti-nerd." Following this division of participants, independent samples $t$ tests were conducted to assess difference in response to a five-point survey item assessing perception of each of five television characters as nerds, with scores of 1 representing the lowest possible nerd identification and scores of 5 representing the highest possible nerd identification. Overall means on this measure ranged from 2.91 (Chuck; $S D=.98)$ to 3.78 (Urkel; $S D=1.01)$, as illustrated in full in Table 3. Mean assessments of character nerdiness by anti-nerd and pro-nerd participants differed significantly in regards to Chuck $(t(380)=-5.32, p<.001)$, Sheldon $(t(377)=-4.72, p<.001)$, Urkel $(t(383)=-2.50, p=.01)$, and Willow $(t(381)=$ $-6.06, p<.001$ ), with pro-nerd participants being more likely in every case to assess each character as being a nerd (see Table 4); no significant difference differences were observed in relation to Milhouse.

These results appear to indicate that individuals who hold positive beliefs and opinions regarding nerds in general are significantly more likely to identify the popular television characters included in this questionnaire as nerds. It is worth emphasizing, however, that even in the anti-nerd condition, mean assessments of nerdiness were close to or above the midpoint of the scale for all five characters, indicating that these 


\section{ARE WE COOL YET?}

characters are indeed perceived as nerds by the majority of audience members. The results of this analysis also illustrate the necessity of controlling for the variable of positive or negative perceptions regarding nerds; consequentially, all subsequent analyses control for this factor by assessing the impact of this variable on statistical results.

Moving forward from this initial assessment, participant responses to questions regarding identification of each of the five television characters as nerds were split along the sample's mean and recoded into a binary nominal variable representing nerd or nonnerd assessment. Subsequently, independent samples $t$ tests were employed, using these newly created grouping variables, in order to assess the extent to which perceptions of characters' mean levels of physical attractiveness, social competence, and technological expertise varied between individuals who did and did not perceive each character as a nerd. (The results of these analyses are illustrated in full in Tables 5 and 6.) These tests indicate, first and foremost, that significant differences in the mean assessments of these variables differed overwhelmingly between individuals who did and did not perceive the characters as nerds. Individuals who perceived a character as a nerd were significantly more likely to assess that character as socially incompetent, physically unattractive, and possessing scientific or technological expertise. Indeed, of the 24 observed cases of statistically significant differences in means, ${ }^{1}$ only one ran in a direction contrary to that which might be expected. This anomalous finding pertained to pro-nerd responses regarding Willow, with mean assessments of physical attractiveness among these respondents being higher for those who also perceived Willow as a nerd than those who did not; this is unusual in that the other characters were assessed as less attractive by 
ARE WE COOL YET?

participants who believed them to be nerds.. With this exception, each character was assessed as less physically attractive by those individuals who perceived that character to be a nerd in at least one of the two conditions (pro- or anti-nerd). Furthermore, assessments of technological and scientific expertise were greater in both conditions for all characters (with the exception of Chuck) among those individuals who perceived each character as a nerd. Lastly, across all characters and in both pro- and anti-nerd conditions, individuals who considered these characters to be nerds provided significantly lower assessments of each character's social competence.

In order to more fully understand the predictive properties of the aforementioned variables upon nerd identification, standard multiple regression analysis was performed for each of the five characters between the dependent variable (identification as a nerd) and independent variables (perceptions of homophily, physical attractiveness, engagingness, social competence, and technological expertise of each character). These analyses were of particular import in the context of this study due to the fact that nerds have rarely if ever been the subject of content analysis, and the conceptualization and operationalization presently employed would resultantly benefit from additional verification. By analyzing the factors which contribute to audience perception of television characters as nerds, it is hoped that regression analysis will provide external validity to the content analysis outlined above, in addition to further illuminating current beliefs and perceptions regarding television portrayals of nerds.

For each of the regression analyses, the study variables outlined above were included in Block 4, while Block 3 included the nerd perception scale previously 


\section{ARE WE COOL YET?}

described, as well as an assessment of whether the respondent was already familiar with the character. Block 2 included media use variables (hours per week of television use and hours per week of Internet use), and Block 1 included demographic variables (age, sex, race, and household income of respondent). Regression analysis revealed that the model significantly predicted audience identification of Chuck character Chuck Bartowski as a nerd, $F(14,250)=7.55, p<.001$. In terms of individual relationships between the independent variables and identification as a nerd, familiarity with the character $(t=-1.97, p=.05)$, perceived social competence in normal interaction $(t=-$ $3.29, p=.001)$ and in intimate interaction $(t=-2.02, p<.05)$ were significant negative predictors of identification as a nerd, with higher scores on these measures predicting a lower likelihood that participants would identify Chuck as a nerd character. By contrast, positive perceptions of nerds in general $(t=5.01, p<.001)$ directly predicted identification as a nerd, with participants who possessed amicable feelings toward nerds being more likely to identify Chuck as such. These effects were observed after controlling for demographic variables and levels of media use, as noted above; the results of this analysis are illustrated in full in Table 7.

Regression analysis revealed that the model described above also significantly predicted audience identification of Simpsons character Milhouse Van Houten as a nerd, $F(14,247)=9.40, p<.001$. In terms of individual relationships between the independent variables and identification as a nerd, perceived physical attractiveness $(t=-2.27, p<$ $.05)$, perceived social competence in intimate interaction $(t=-4.91, p<.001)$, and perceived homophily $(t=-2.00, p<.05)$ were significant negative predictors of 
ARE WE COOL YET?

identification as a nerd. By contrast, perceived engagingness $(t=2.41, p=.01)$, perceived technological expertise $(t=2.98, p<.01)$ positively predicted identification as a nerd. These effects were observed after controlling for demographic variables and levels of media use, as noted above; the results of this analysis are illustrated in full in Table 8.

Regression analysis revealed that the model described above also significantly predicted audience identification of The Big Bang Theory character Dr. Sheldon Cooper as a nerd, $F(14,251)=18.73, p<.001$. In terms of individual relationships between the independent variables and identification as a nerd, perceived social competence in normal interaction $(t=-2.83, p<.01)$ and in intimate interaction $(t=-3.84, p<.001)$, as well as perceived homophily $(t=-2.65, p<.01)$ were significant negative predictors of identification as a nerd. By contrast, perceived technological expertise $(t=4.86, p<$ .001) positively predicted identification as a nerd. These effects were observed after controlling for demographic variables and levels of media use, as noted above; it was noted that positive perceptions of nerds in general also acted as a statistically significant positive predictor of identification of Sheldon as a nerd, $t=3.63, p<.001$. The results of this analysis are illustrated in full in Table 9.

Regression analysis revealed that the model described above also significantly predicted audience identification of Family Matters character Steve Urkel as a nerd, $F(14,251)=13.74, p<.001$. In terms of individual relationships between the independent variables and identification as a nerd, perceived physical attractiveness $(t=-$ $2.65, p<.01)$ and perceived social competence in intimate interaction $(t=-3.82, p<$ 


\section{ARE WE COOL YET?}

.001) were significant negative predictors of identification as a nerd. By contrast, perceived technological expertise $(t=7.48, p<.001)$ positively predicted identification as a nerd. These effects were observed after controlling for demographic variables and levels of media use, as noted above. It was noted, however, that participant level of household income also acted as a statistically significant positive predictor of identification of Urkel as a nerd $(t=2.39, p<.05)$, while prior familiarity with the character negatively predicted such identification $(t=-1.92, p=.05)$. The results of this analysis are illustrated in full in Table 10.

Regression analysis revealed that the model described above also significantly predicted audience identification of Buffy the Vampire Slayer character Willow Rosenberg as a nerd, $F(14,249)=9.39, p<.001$. In terms of individual relationships between the independent variables and identification as a nerd, perceived social competence in normal social interaction $(t=-2.14, p<.05)$ and in intimate interaction $(t$ $=-3.79, p<.001)$ were significant negative predictors of identification as a nerd. By contrast, perceived technological expertise $(t=2.32, p<.05)$ positively predicted identification as a nerd. These effects were observed after controlling for demographic variables and levels of media use, as noted above. It was noted, however, that participant sex also acted as a statistically significant predictor of identification of Willow as a nerd $(t=-1.95, p=.05)$, with men being less likely than women to assess this character as nerd. Additionally, prior familiarity with the character negatively predicted identification as a nerd $(t=-2.32, p<.05)$, while positive perceptions of nerds in general 
ARE WE COOL YET?

was a powerful direct predictor of such identification $(t=4.16, p<.001)$. The results of this analysis are illustrated in full in Table 11.

Holistically, the results of these regression analyses lend substantial support to the conceptualization and operationalization of nerds employed in the content analysis portion of this study. Technological expertise was a frequent positive predictor of nerd identification, while physical attractiveness and social competence emerged only as negative predictors of such identification. In each case, the model outlined above proved a good fit for the prediction of television characters as nerds, with each of the study variables relating to nerd identification in the direction predicted. Additionally, these data indicate that individuals who are positively inclined toward nerds in general are also more likely than negatively-inclined individuals to perceive characters on television as nerds. Given this, it is interesting to note that perceptions of homophily with television characters only rarely impacted perceptions of those characters as nerds; still more interesting is the fact that when this variable emerged as a significant predictor, it was negatively associated with nerd identification. It might be expected that self-identified nerds would be eager to identify the television characters with whom they identify as being nerds themselves; perhaps, however, the opposite is true for non-nerd viewers, with such viewers perceiving the characters as similar to themselves but denying the nerdiness of these characters. Having established the validity of social competence and physical attractiveness as negative predictors of nerd identification, analysis was conducted which addressed the research questions and hypotheses associated with these variables.

\section{Social Competence}




\section{ARE WE COOL YET?}

In order to provide a baseline measurement against with public perception and variance by sex might be contrasted, a one-way analysis of variance was employed using the year groups described above. This ANOVA was constructed to assess the degree to which the social competence of nerds in popular broadcast television programs has changed over the course of the past twenty years (RQ1b). Analysis of these year groups revealed no statistically significant differences in mean levels of social competence between groups, $\left.F(4,73)=.93, p=.44, \eta^{2}=.04\right)$. The variance between the groups of 1992 to $1995(M=2.78, S D=1.64), 1996$ to $1999(M=3.07, S D=1.87), 2000$ to 2003 $(M=2.29, S D=1.20), 2004$ to $2007(M=1.92, S D=1.25)$, and 2008 to $2011(M=2.48$, $S D=1.92$ ) was minimal. Portrayals of nerds in popular broadcast television programs appear to have remained relatively constant in their exhibited levels of social competence, with nerd characters exhibiting 0 to 8 instances of socially inappropriate behavior during each episode in which they appeared $(M=2.5)$. As noted earlier, this relatively low mean appears to indicate that nerd characters in this study's sample were, apparently, only moderately incompetent. Without an assessment of non-nerd characters, the relative significance of this level of social (in)competence is difficult to determine; it is safe to say, however, that the mean level observed during content analysis was by no means extraordinarily high.

While no significant differences in means were evident in the results of the initial analysis, it was noted during the course of this analysis that the year groups during which nerd characters exhibited somewhat greater degrees of social competence appeared to coincide with those year groups during which nerd characters appeared more frequently 
ARE WE COOL YET?

in dramatic television programs. In order to explore the potential disparity in portrayals between programming types, an independent samples $t$ test was employed to compare mean levels of social competence exhibited by nerds in comedic television programs and dramatic television programs. This analysis revealed that the degree of social competence exhibited by nerd characters in comedic programs $(n=53, M=2.87, S D=$ 1.66) differed significantly from that exhibited by nerd characters in dramatic programs $(n=25, M=1.72, S D=1.42), t(76)=2.96, p<.01$. Thus, nerd characters are portrayed as significantly more lacking in social competence in comedic television programs than in dramatic television programs.

In light of the clearly demonstrated disparity between male and female portrayals of nerds, an independent samples $t$ test was employed to assess the degree to which mean levels of social competence exhibited by nerds in popular television programs may vary on the basis of character sex (RQ3). This analysis revealed that the degree of social competence exhibited by male nerd characters $(n=70, M=2.56, S D=1.66)$ did not differ significantly from the degree of social competence exhibited by female nerd characters $(n=8, M=2.00, S D=1.77), t(76)=.89, p=.37$. This lack of statistical significance may, however, be due in part to the substantially smaller number of female characters identified as nerds in the study sample. It is possibly, or even likely, that the difference in mean levels of social competence would exhibit statistical significance with a greater sample of female nerd portrayals.

Further analysis was conducted of mean levels of television nerds' social competence as perceived by survey participants (RQ2b), with Pearson product moment 
ARE WE COOL YET?

correlation employed to explore the extent to which these perceptions corresponded with perceptions of nerds in real life. In order to address variance in perceptions across individuals on the basis of positive or negative perceptions of nerds, this analysis was conducted with responses split along the mean response point with respect to nerd perceptions. Responses were gathered through a two-item measure assessing perceived social competence of each of five television characters, with scores of 1 representing the lowest possible social competence and scores of 5 representing the highest possible social competence. Cronbach's $\alpha$ for this measure was .78 for Chuck, .77 for Milhouse, .77 for Sheldon, .68 for Urkel, and .80 for Willow. Mean scores on this measure ranged from 2.20 (Sheldon; $S D=.97)$ to $2.96($ Chuck; $S D=.82)$ for the pro-nerd condition and from 2.61 (Milhouse; $S D=1.00$ ) to 3.25 (Willow; $S D=.95$ ) for the anti-nerd condition. (The results of this analysis are illustrated in full in Table 12).

It is intriguing to note that in each case, characters were assessed as more socially competent by those in the anti-nerd condition than those in the pro-nerd condition. Furthermore, in the anti-nerd condition, assessments of the social competence of nerd characters was found to be positively correlated to perceptions of social competence of real-life nerds in the case of Sheldon, $r(212)=.33, p<.001$, and Urkel, $r(212)=.33, p<$ .001 . Interestingly, in the pro-nerd condition, perceptions of social competence of reallife nerds did not correlate significantly with assessments of social competence of any of the television characters analyzed. These findings are particularly noteworthy in light of the results of the independent samples $t$ tests outlined earlier which explored differences in mean levels of assessed social competence between participants who did and did not 


\section{ARE WE COOL YET?}

perceive characters to be nerds. While the results of these $t$ tests suggested that perceived social competence was significantly lower among individuals who believed characters to be nerds, the correlations conducted here appear to indicate a disconnect between the perceptions of nerds on television and in real life. In order to more completely explore this dynamic, a post hoc analysis was conducted employing an independent samples $t$ test to assess pro- and anti-nerd participants' perceptions regarding social competence of nerds in general. This test revealed that the mean perceived levels of the social competence of nerds in general among participants with pro-nerd leanings $(M=2.65, S D$ $=.89)$ were higher than those of participants with anti-nerd leanings $(M=2.31, S D=$ $.83)$, with this difference being statistically significant, $t(384)=-3.88, p<.001$. Thus, even noting the disconnect described above, it seems that individuals who view nerds positively are likely also to assess them as more socially competent.

\section{Physical Attractiveness}

As a final step in the evaluation of over-time trends in nerd portrayals, a one-way analysis of variance was conducted to assess the degree to which the physical attractiveness of nerds in popular broadcast television programs has changed over the course of the past twenty years (RQ1c). In keeping with the previous analyses, comparisons were made between mean levels of physical attractiveness exhibited by nerd characters observed during the year groups outlined above. This analysis indicated significant differences in mean levels of physical attractiveness between year groups, $F$ $\left.(4,73)=5.83, p<.001, \eta^{2}=.24\right)$. To assess pairwise differences between the year groups, the Scheffe post-hoc procedure $(p=.05)$ was performed. The results indicated 
ARE WE COOL YET?

that mean levels of physical attractiveness exhibited by nerd characters in the 1996 to 1999 group $(M=1.93)$ were significantly lower than those of nerd characters in the 2000 to $2003(M=3.14)$ and 2008 to $2011(M=3.19)$ groups. Thus, while no significant difference in means was observed in relation to the 1992 to $1995(M=2.33, S D=.70)$ or 2004 to $2007(M=2.92, S D=1.11)$, this analysis provides evidence that the physical attractiveness of nerd characters has changed significantly, particularly since the turn of the millennium. Portrayals of nerds observed in programs aired during eight of the last twelve years included in this sample were significantly more physically attractive than those in programs aired in the four years prior to 2000. Additionally, while there was no significant difference between the later year groups and the earliest group (1992 to 1995), it is worth noting that only nine nerd characters were observed within the latter group. Given the low mean level of physical attractiveness during this timeframe, it is likely that statistical significance would have been achieved had a greater number of nerds been observed.

As with the analysis of social competence described above, it was noted during the course of this analysis that the year groups during which nerd characters exhibited greater degrees of physical attractiveness appeared to coincide with those years during which nerd characters appeared more frequently in dramatic television programs. In order to explore the potential disparity in portrayals between programming types, an independent samples $t$ test was employed to compare mean levels of physical attractiveness exhibited by nerds in comedic television programs and dramatic television programs. This analysis revealed that the degree of physical attractiveness exhibited by 


\section{ARE WE COOL YET?}

nerd characters in comedic programs $(n=53, M=2.55, S D=.88)$ differed significantly from that exhibited by nerd characters in dramatic programs $(n=25, M=3.32, S D=$ 1.06), $t(76)=-3.35, p=.001$. Nerd characters were portrayed as significantly less attractive in comedic television programs than in dramatic television programs.

In order to lend greater depth to these findings, public perception of television nerds' physical attractiveness was assessed (RQ2c), with Pearson product moment correlation employed to explore the extent to which these perceptions correspond with perceptions of nerds in real life. As with the evaluation of perceived social competence outlined above, this analysis was conducted with responses split along the mean response point with respect to nerd perceptions in order to address differences between pro-nerd and anti-nerd respondents. Responses were gathered through a five-point questionnaire item assessing perceived physical attractiveness of each of five television characters, with scores of 1 representing the lowest possible attractiveness and scores of 5 representing the highest possible attractiveness. Mean scores on this measure ranged from 2.37 (Milhouse; $S D=1.13$ ) to 4.02 (Willow; $S D=.99$ ) for the pro-nerd condition and from 2.20 (Urkel; $S D=.93$ ) to 3.88 (Willow; $S D=1.01$ ) for the anti-nerd condition. (The results of this analysis are illustrated in full in Table 13.)

As can be seen, with the exception of Milhouse, all characters were assessed as more physically attractive by pro-nerd respondents than by those in the anti-nerd group. Furthermore, in the anti-nerd condition, assessments of the physical attractiveness of nerd characters was found to be positively correlated to perceptions of social competence of real-life nerds in the case of Sheldon, $r(211)=.22, p=.001$, and Urkel, $r(210)=.16, p=$ 
ARE WE COOL YET?

.01. In the pro-nerd condition, however, only in the case of Sheldon was the correlation between assessed physical attractiveness and perceptions of the physical attractiveness of nerds in general found to be significantly and positively correlated, $r(172)=.27, p<$ .001. As discussed above in regards to perceptions of social competence, these analyses appear to indicate that perceptions of the physical attractiveness of nerd characters on television are not necessarily related to the perceptions individuals might hold regarding the physical attractiveness of nerds in real life. In order to more completely explore this dynamic, a post hoc analysis was conducted employing an independent samples $t$ test to assess pro- and anti-nerd participants' perceptions regarding physical attractiveness of nerds in general. This test revealed that the mean perceived levels of the physical attractiveness of nerds in general among participants with pro-nerd leanings $(M=3.76$, $S D=1.02)$ were higher than those of participants with anti-nerd leanings $(M=2.51, S D=$ 1.02), with this difference being highly significant, $t(383)=-11.92, p<.001$. Thus, while the disconnect observed earlier with respect to social competence is once again evident, it is also clear that individuals who view nerds positively are likely also to be more generous in their assessment of nerds' physical attractiveness.

To ensure the comprehensiveness of statistical analyses, independent samples $t$ tests were employed to compare mean levels of perceived physical attractiveness of each television character on the basis of respondent sex. These analyses were undertaken in order to assess potential discrepancies between assessments by male or female respondents in regards to male or female characters. The results of these $t$ tests indicate that no significant differences exist between male and female assessments of the physical 


\section{ARE WE COOL YET?}

attractiveness of nerd characters in the anti-nerd condition. In the pro-nerd condition, however, participants' mean assessments differed significantly for males $(M=2.65, S D=$ $1.15)$ and females $(M=2.05, S D=1.02)$ regarding Milhouse, $t(170)=-3.53, p=.001$, and for males $(M=4.18, S D=.81)$ and females $(M=3.88, S D=1.11)$ regarding Willow, $t(169)=1.95, p=.05$, with two of the other three cases approaching statistical significance. These analyses are illustrated in full in Tables 14 and 15; in sum, it appears that viewer sex is only a factor in evaluations of character attractiveness when the viewer possesses positive feelings regarding nerds in general. Furthermore, while it was expected that female viewers would assess male characters as more attractive and male viewers would assess female characters as more attractive, the opposite was shown to be the case with respect to four out of five of the characters, with Chuck being the only exception.

As a final analysis of sex-based disparities among nerd portrayals, an independent samples $t$ test was employed to test the hypothesis $(\mathrm{H} 2)$ that mean levels of physical attractiveness exhibited by nerds in popular television programs will vary, with female characters exhibiting greater levels of physical attractiveness than male characters. This analysis revealed that the degree of physical attractiveness exhibited by male nerd characters $(n=70, M=2.67, S D=.94)$ differed from that exhibited by female nerd characters $(n=8, M=3.88, S D=.99)$, and that this difference in means was statistically significant, $t(76)=-3.40, p=.001$. Female nerd characters exhibited substantially greater levels of physical attractiveness than did male nerd characters; thus, hypothesis two was supported. The level of statistical significance achieved by this difference in means is 


\section{ARE WE COOL YET?}

particularly noteworthy in light of the small number of female nerd characters upon which this analysis is based.

The holistic results of these statistical analyses supply a relatively detailed overview of nerd portrayals in popular television, as well as audience perceptions of such portrayals in conjunction with perceptions of nerds in real life. The number of nerd characters in popular television programs is significantly lower than the number of nonnerd characters; it has remained relatively consistent over the past 20 years, with 2011 representing the only observed spike in frequency. Nerds remain almost exclusively white and male; they are generally portrayed (and perceived) as socially incompetent and physically unattractive, although female nerds tend to be more attractive than their male counterparts and nerds in dramatic programs are generally more attractive and competent than are portrayals in comedic programs. Lastly, and arguably most importantly, the traits and characteristics exhibited by nerds observed in the course of content analysis of popular television programs were found to be mirrored in much of the public opinion survey data. These data provide a robust foundation upon which to build a discussion of the theoretical and sociocultural implications of the study outlined above.

\section{Notes}

${ }^{1}$ : Independent samples $t$ test syntax - Pro-nerd assessments of social competence of Chuck: $t(169)=3.89, p<.001$. Pro-nerd assessments of physical attractiveness of Milhouse: $t(167)=1.91, p=.05$. Pro-nerd assessments of social competence of Milhouse: $t(168)=6.20, p<.001$. Pro-nerd assessments of technological $/$ scientific 


\section{ARE WE COOL YET?}

expertise of Milhouse: $t(164)=-5.38, p<.001$. Pro-nerd assessments of social competence of Sheldon: $t(166)=6.65, p<.001$. Pro-nerd assessments of technological/scientific expertise of Sheldon: $t(161)=-8.73, p<.001$. Pro-nerd assessments of social competence of Urkel: $t(170)=3.96, p<.001$. Pro-nerd assessments of technological expertise of Urkel: $t(167)=-7.67, p<.001$. Pro-nerd assessments of physical attractiveness of Willow: $t(169)=-4.10, p<.001$. Pro-nerd assessments of social competence of Willow: $t(171)=4.48, p<.001$. Pro-nerd assessments of technological expertise of Willow: $t(170)=-5.13, p<.001$. Anti-nerd assessments of physical attractiveness of Chuck: $t(207)=3.80, p<.001$. Anti-nerd assessments of social competence of Chuck: $t(209)=3.84, p<.001$. Anti-nerd assessments of physical attractiveness of Milhouse: $t(208)=2.86, p=.005$. Anti-nerd assessments of social competence of Milhouse: $t(207)=7.57, p<.001$. Anti-nerd assessments of technological/scientific expertise of Milhouse: $t(207)=-3.71, p<.001$. Anti-nerd assessments of physical attractiveness of Sheldon: $t(206)=2.55, p=.01$. Antinerd assessments of social competence of Sheldon: $t(209)=10.04, p<.001$. Anti-nerd assessments of technological/scientific expertise of Sheldon: $t(207)=-4.54, p<.001$. Anti-nerd assessments of physical attractiveness of Urkel: $t(208)=5.43, p<.001$. Antinerd assessments of social competence of Urkel: $t(211)=5.16, p<.001$. Anti-nerd assessments of technological expertise of Urkel: $t(208)=-4.97, p<.001$. Anti-nerd assessments of social competence of Willow: $t(208)=3.71, p<.001$. Anti-nerd assessments of technological expertise of Willow: $t(205)=-2.55, p=.01$. 


\section{ARE WE COOL YET?}

\section{Chapter 5: Discussion}

The purpose of the analyses of television programming and public opinion undertaken in this study was to explore current and recent trends in the portrayals of nerds in popular broadcast television programs, as well as the potential intersection thereof with commonly-held perceptions of nerds on television and in real life. Although the past several years have seen substantial attention devoted to the "birth of the nerd" (Zimmer 2011, p. 1) and the increasing popularity of nerds within the mainstream media (e.g., Bricken \& Heiler, 2009; Chruscinski, 2008, Liberman, 2007; and Stice, 2011), quantitative research regarding the actual mediated representations of nerds has thus far been minimal. Building upon the foundation laid by past media scholars (Conway \& Rubin, 1991; Gerbner \& Gross, 1976; Gerbner, 1998; Hall, 1973; Lippmann, 1922; McCombs \& Shaw, 1972; McLuhan \& Fiore, 1967; Smythe, 1954), this study aims to provide a framework for future quantitative and critical exploration of the representation of nerds and the potential implications of these representations. The following section provides a summary of the findings resultant from the statistical analyses undertaken in the course of this study, as well as limitations to this study and suggestions for further lines of scholarly inquiry.

\section{Summary of Findings}

Nerds in general. The first research question addressed the extent to which portrayals of nerds in popular broadcast television programs have or have not changed over the course of the past twenty years. Interestingly, an analysis of the top five most popular programs from each of these years indicates that the frequency with which nerd 
ARE WE COOL YET?

characters appear in popular broadcast television shows has remained relatively stable, with nothing approaching a statistically significant increase appearing evident. It is worth noting, however, that the number of nerd characters observed in the television program episodes drawn from the 2011 broadcast year was nearly three times that observed in any other broadcast year. While the year groups employed in subsequent analysis rendered the statistical significance of this discrepancy difficult to assess, it is possible that this sudden increase may prove to be indicative of a lasting increase in nerd portrayals in popular television. As noted throughout this paper, nerds and nerd culture have increasingly been the subjects of attention by the mass media within the past several years. If the upswing in nerd characters observed during the 2011 broadcast year proves permanent rather than temporary, with such portrayals becoming a ubiquitous part of the television broadcasting landscape, it is possible that what has to this point been a nerd subculture may begin to move from the margins to the center of American society. The regression analyses conducted in this study indicate that technological and computerrelated expertise remain significant predictors of identification as a nerd. Given this, in conjunction with the ever-increasing premium placed upon these proficiencies, television viewers may perceive increased nerd portrayals as an indication that such brainy pursuits are in fact something to which they should aspire.

If the coming years do in fact bear witness to a sustained increase in nerd characters in popular television programs, the question becomes, then, one of critical social importance - which audience members will see themselves reflected on the screen? The first hypothesis addressed the assertion that characters identified as nerds in popular 
ARE WE COOL YET?

broadcast television are more likely to be portrayed as male than as female. This assertion is based upon substantial work within both scholarly circles and the popular press, the vast majority of which indicates that many of the characteristics generally considered indicative of nerd identification (particularly technological and computerrelated expertise) remain associated with masculinity. As expected, the characters identified as nerds in course of this study's content analysis were overwhelmingly male as opposed to female, lending still greater support to this study's rationale.

It must also be emphasized that this discrepancy is far from minor; nearly nine out of ten of the characters identified as nerds were male, with only eight out of the 78 nerd characters being female. This is not an insignificant trend which can be written off as merely a statistical anomaly or a result of the type of sample drawn. Even as the presence of nerds in popular television increases in frequency, with these nerds becoming increasingly attractive and audience members finding them engaging and enjoyable to watch, female television characters remain systematically excluded from this subcultural category. While television and other mass media are not the sole means by which individuals develop their beliefs and perceptions regarding social mores and cultural expectations, the work of past and contemporary media scholars indicates that consistent representation (or lack of representation) plays a role in constructing and enforcing such perceptions. An increasing recognition and acceptance of nerds in television (and, consequently, American culture as a whole) will do little to advance the inclusion of girls and women in mathematic, scientific, and technological fields if these nerds are portrayed as almost exclusively male. Even the discrepancy observed between male and female 
ARE WE COOL YET?

nerd portrayals, however, was not as large as that observed in regards to race; of the 78 characters identified as nerds, only six (a mere 7.7\%) were coded as non-Caucasian. As was predicted on the basis of a review of extant literature and scholarship, rather than destabilizing or disrupting the hegemonic framework which associates scientific and technological expertise with whiteness, the nerd identity continues to be conceptualized and employed as a signifier of such whiteness.

In order to more fully explore the sociocultural implications of nerd portrayals in the media, the second research question addressed the perceptions and beliefs held by the general public in regard to nerds in popular television programs. The results of the analyses employed in the exploration of these perceptions indicate, first and foremost, that members of the general public were over six times more likely to state that nerds are predominately male than to state that they are predominately female. As with the disparity between male and female nerds in popular television programs in terms of frequency, the disparity in perceived gender-coding of nerd identity was overwhelming. While the construction of the survey questionnaire prevents any assessment of statistical significance in regards to this single item, these findings clearly illustrate the need for research exploring viewer perceptions of the gendered nature of nerds as a subcultural category.

Additionally, and perhaps more importantly for the validation of the measures employed in this study, analysis of public opinion data indicates that beliefs regarding the lack of social competence and physical attractiveness remain a persistent factor in the determination of which individuals are labeled as nerds. Multiple regression analysis, 
ARE WE COOL YET?

controlling for demographic variables, levels of media use, and perceptions of nerds, repeatedly indicated that both perceived social competence and perceived physical attractiveness were significant negative predictors of participants' identification of television characters as nerds. Perceived technological expertise proved to be a significant positive predictor of such identification, while perceived homophily served as a negative predictor. This was surprising, given the fact that positive perceptions of nerds in general were, more often than not, powerful positive predictors of nerd identification, and it would thus stand to reason that the individuals who would be proud to be considered nerds might be more likely to identify these characters as such. Other control and study variables sporadically achieved statistical significance as predictors, but it was these five variables which emerged as exerting the strongest and most consistent impact upon audience perceptions of characters as nerds.

These results are worthy of discussion for a number of reasons. First, they lend significant support to the conceptualization and operationalization of nerds undertaken in the context of this study, as the relationships between social competence, physical attractiveness, and nerd identification appear as robust as was expected after a comprehensive review of extant literature and popular press publications. Second, on a related point, while the aforementioned content analysis of popular television programming indicates that nerd characters have increased in physical attractiveness, unattractiveness remains a characteristic which is strongly associated with nerds by the general public. This apparent contradiction raises questions regarding the extent to which attractive characters who exhibit other nerd characteristics are in fact identified as nerds 
ARE WE COOL YET?

by audience members; further research is necessary to ascertain the nature of this particular anomaly. Third, it is particularly of interest to the researcher that perceived homophily repeatedly emerged as a statistically significant negative predictor of nerd identification. This finding, in conjunction with the increased attention devoted to nerds by the popular press and the increased frequency with which nerds have appeared in popular broadcast television shows in the past four years, raises questions concerning the significance assigned by the general public to what have thus far been considered "negative" traits. Perhaps it is possible that even as nerds have increased in recognition and exposure, viewers continue to draw distinctions between the characters portrayed in popular television programs and their own self-conceptualizations and identities. Having established the validity of social competence and physical attractiveness as inverse predictors of nerd identification, the following subsections outline the findings pertinent to these variables.

\section{Social competence.}

As with frequency of nerd portrayals in popular television programs, the data collected in the course this study indicate that the social competence of these characters has remained relatively stable over the past twenty years. Furthermore, examination of the relationship between perceptions of nerd characters' social competence with beliefs regarding the social competence of real-life nerds revealed significant correlations in only two of the ten analyses conducted, indicating individuals may have different expectations of nerds on television and in real life. What is perhaps more interesting is the fact that both these significant correlations were uncovered in the analysis of participants who 
ARE WE COOL YET?

held more negative views regarding nerds in general. Additionally, and particularly surprisingly, participants possessing negative views regarding nerds were more likely to perceive the television characters included in this analysis as socially competent than were those participants with positive views regarding nerds. These findings were outweighed, however, by the fact that in every one of the analyses conducted, individuals who perceived a given character to be a nerd were, without exception, more likely to assess that character's social competence as lower than they would if that character was not considered a nerd.

These findings run contrary to what might be expected; intuitively, it seems that individuals with more positive feelings toward nerds in general would be likely to assess nerd characters on television more positively (i.e., as more socially competent) than would individuals with negative feelings toward nerds. It is interesting to note that the two characters for whom a statistically significant correlation was found between character assessments and audience beliefs regarding real-life nerds were Steve Urkel and Sheldon Cooper. These two characters were the characters most frequently (indeed, nearly universally) cited as nerds in the popular press articles from which the characters included in this study were drawn. Given the findings outlined above regarding predictors of identification of characters as nerds, it is perhaps unsurprising that the characters who are assessed as most clearly exhibiting the characteristics viewers continue to associate with nerds will be correspondingly more likely to correlate with audience perceptions regarding nerds in real life. It should also be noted that these characters earned among the lowest assessments of social competence by participants; 
ARE WE COOL YET?

thus, it is possible that the correlation which was observed may be a function of the relative strength or forcefulness of audience perceptions.

The third and final research question addressed the possibility that nerd characters might vary in their level of social competence on the basis of sex; both male and female nerd characters acted in a manner considered by other characters to run contrary to social convention an average of two to three times per episodes. Non-nerd characters were not coded for variables such as social competence, and comparisons between groups are consequentially impossible; however, it seems plausible that the average nerd character's level of social competence may be lower than that of the average non-nerd character. As will be discussed below, distinct differences do indeed exist between male and female portrayals of nerds in popular broadcast television programming, but it appears possible that the lack of social competence may remain a unifying factor regardless of sex. Equally likely, however, is the possibility that significant differences may exist, but the number of female nerds (8) observed in this study's sample was insufficient to illustrate any such disparities; further research is necessary to address this potentiality.

\section{Physical attractiveness.}

The study's analyses of the means levels of physical attractiveness of nerds portrayed in popular broadcast television programs yielded somewhat surprising results. Whereas no significant differences were observed across year groups with respect to levels of social competence, the mean levels of physical attractiveness exhibited by these characters was significantly greater in two of the later year groups than in the "1997 to 2000" group. Nerds in popular television programs have, seemingly, grown (at least 
ARE WE COOL YET?

somewhat) more handsome and beautiful, but this increase has not brought with it a concomitant shift in social competence. This is on the one hand unsurprising, as there is no reason to expect the variables of social competence and physical attractiveness to act as covariates; nonetheless, it might be expected that the characteristics exhibited by nerds on television would become holistically more positive or negative. Such does not, however, appear to be the case, which may indicate that social incompetence - a trait which has remained constant in nerd portrayals across the twenty years included in this study - is considered a more central and necessary prerequisite to nerdiness than is physical unattractiveness.

As with the analysis of social competence and physical attractiveness over time, survey data regarding public opinion and perception of nerds' physical attractiveness yielded results distinctly different from those pertaining to social competence. Whereas participants who possessed positive feelings toward nerds in general were less likely to assess nerd characters as socially competent, the opposite was found to be the case in regards to physical attractiveness. Analyses of perceptions of physical attractiveness indicated that those participants with pro-nerd leanings were more likely to assess nerd characters (with the exception of Milhouse) as attractive than were those with anti-nerd leanings. As with social competence, however, correlation analyses yielded only weak evidence that perceptions of nerd characters' physical attractiveness are linked to beliefs regarding the physical attractiveness of nerds in real life. There appears to be a disconnect of some sort between perceptions of the physical attractiveness of nerd characters on television, even when individuals explicitly state their belief that a given 


\section{ARE WE COOL YET?}

character is a nerd, and beliefs regarding the physical attractiveness of nerds in real life. It must be emphasized again that the characters included in the questionnaire were not drawn at random, but were selected on the basis of their appearance in lists of top television nerds; thus, they may be considered highly similar to the portrayals of nerds observed in the course of content analysis. In the case of four out of five of these characters (with Willow being the exception), individuals who perceived each character as a nerd were likely to assign that character significantly lower levels of physical attractiveness; given this, it seems highly likely that such portrayals reinforce existing beliefs and stereotypes regarding nerds.

More interestingly still, the results of these analyses indicate that male viewers tend to assign greater levels of physical attractiveness to most male nerd characters than do female viewers, while female viewers tended to perceive Willow (the only female nerd character included in analysis) as more physically attractive than did male viewers. These disparities were only statistically significant among viewers with positive perceptions of nerds in general; however, the implications are intriguing to consider. It is possible, based on these data, that participants do not feel it is appropriate or "cool" to express attraction for television characters considered to be nerds, and that it is therefore more likely for individuals of the same sex to feel less threatened by the idea of assessing such characters as physically attractive. This relationship may be mediated by the variable of sexual orientation, which was not assessed in this study's questionnaire; nonetheless, it poses an interesting possibility. 


\section{ARE WE COOL YET?}

The second and final hypothesis included in this study addressed the assumption that female nerd characters will exhibit a greater degree of physical attractiveness than will male nerd characters. Substantial research has previously documented the sexualization and objectification of women in the mass media, and it seems likely that the tendency of nerd portrayals to minimize physical attractiveness would not be sufficient to outweigh this trend. The results of the analysis employed to test these assumptions were definitive in that the physical attractiveness of nerd characters differed strongly and significantly on the basis of sex, with this disparity being observed in precisely the directionality predicted by the researcher. Female nerd characters were significantly more likely to be portrayed as physically attractive than were male nerd characters. As touched upon earlier, the level of statistical significance achieved by this analysis of difference in means is particularly striking in light of the small number of female nerd characters included in this study's sample. It is likely that future studies including a greater number of female nerd characters will provide still stronger evidence of this disparity.

In any event, the portrayal of female nerd characters as physically attractive holds both encouraging and problematic implications. It is true that greater levels of physical attractiveness may result in an increased likelihood that female viewers will perceive female nerds as cool, positive portrayals worthy of emulation. This possibility, however, does not excuse the hypersexualization and objectification of women which remains all too common in the mass media. As noted above, in addition to assessing the physical attractiveness of nerd characters, each character's body shape was also coded through the 


\section{ARE WE COOL YET?}

use of a nine-point measure; given the aforementioned hypersexualization of women in the mass media, male and female characters were compared on the basis of this variable. An independent samples $t$ test between indicated that male nerd characters $(M=5.00, S D$ $=1.73)$ differed from female characters $(M=3.75, S D=1.16)$, with female characters being significantly thinner than male characters, $t(76)=1.98, p=.05$. These results provide still stronger evidence that even on the rare occasions when nerds are portrayed as female, such portrayals must still exemplify the physical attributes deemed desirable by the makers and consumers of the media. Again, while it may be true that attractive female portrayals of nerds are less likely to "scare off" women and girls considering a career in science or technology (Spertus, 1998), such portrayals do nothing to encourage a culture of respect and valuation for those of them who do choose to enter such careers. No number of female nerd characters will serve to foster progressive and inclusive social change if the focus of such characters is their physical attributes and sexuality. A female scientist or mathematician whose intellectual and academic prowess is overshadowed by her exaggerated bust and abbreviated hemline does little to encourage women and girls to believe that a place is waiting for them as equal and respected members of the scientific or technological community.

It is also interesting to note that while time has not rendered significant changes to the levels of social competence exhibited by nerd characters, the post hoc comparison of mean levels of physical attractiveness and social competence exhibited by nerd characters in comedic and dramatic programs revealed that these portrayals did in fact differ significantly. While this is in one sense seemingly intuitive, as the pratfalls and general 


\section{ARE WE COOL YET?}

awkwardness associated with unattractive and socially incompetent characters are more well-suited to comedic than dramatic settings, it is nonetheless interesting to note the statistical strength of these differences. The distinctions between nerds in comedic and dramatic programs are also perhaps symptomatic of what has been referred to as the "Hollywood Nerd" syndrome (tvtropes.org, n.d.).

In numerous movies and television programs, particularly in recent years, characters who would otherwise not be identifiable as nerds on the basis of conceptualizations such as those employed in the current study have been given certain physical markers (such as glasses or lab coats) and hobbies traditionally associated with nerds (such as comic books or knowledge of science fiction). These characters are generally quite attractive and, while occasionally teased by others due to their affinity for "nerdy" pursuits, tend to exhibit far fewer signs of social awkwardness than do more traditional nerd characters. It seems possible, or even likely, that the nerd characters portrayed in dramatic television programs are likely to fall within this category. Indeed, during the course of content analysis, the researcher identified numerous characters (particularly in dramatic programming) who epitomized the concept of the Hollywood nerd, but the majority of these characters exhibited insufficient markers of nerd behavior to be included in the final analysis.

The disparity between nerds in comedic and dramatic programs becomes more significant still when program length is considered. Many of the dramatic television programs included in this analysis were twice the length of most of the comedic programs; this is particularly noteworthy due to the operationalization of social 


\section{ARE WE COOL YET?}

competence as an additive index, as the greater program length might be expected to lead to correspondingly higher levels of social (in)competence. This was not the case, however; indeed, as described above, nerd characters in dramatic programs exhibited more social competence than their counterparts in comedic programs, in spite of the difference in program length. To the researcher's knowledge, little if any inquiry has been undertaken to explore the phenomenon of the Hollywood nerd; the results of this study, however, would appear to indicate that such inquiry is unquestionably warranted. These results also provide further support for contentions that different genres of media text may serve distinct communicative purposes and ideological functions, and further, that each genre may draw upon disparate sociocultural stereotypes and archetypes in the pursuit of these functions (Bednarek, 2011; Askehave \& Nielsen, 2005). Based upon these results, it can be unequivocally stated that future examinations of nerds in the mass media must remain cognizant of the impact and implications of genre in regards to the characterizations employed in the course of each media text.

\section{Theoretical Implications}

The dual study designs employed in the course of this exploration of nerd portrayals yielded a complex and multifarious dataset, the analysis of which raises numerous intriguing possibilities regarding potential theoretical and practical implications. First and foremost, this study was predicated upon the belief that nerds, and the identification as such, have increasingly come into vogue within American culture, but that women have been largely excluded from such identification within the mass media. While the former point was only tenuously illustrated in the data resultant from 
ARE WE COOL YET?

this study, the latter was proven to be overwhelmingly the case. As has been previously discussed, nerds are generally perceived as being physically unattractive and socially incompetent, a perception which, based on the regression analyses outlined above, appears evident in the responses of this study's survey participants. The results of the content analysis undertaken in this study further serve to support these assertions, leading to the possibility of cyclical reinforcement between audience beliefs and perceptions and portrayals of nerds in popular television. Additionally, however, nerds are often believed to possess exceptional expertise in regards to computers, technology, or both; again, the results of regression analysis lend empirical support to the statement of these perceptions. Given this perception, in conjunction with the premium increasingly placed on such expertise, the massive discrepancy observed during content analysis between male and female nerds in popular television programs is problematic, to say the least.

Numerous media scholars, notably Gerbner (1998), Lippmann (1922), and Conway and Rubin (1991), have argued that the images presented in the mass media are among the most powerful tools by individuals craft their beliefs and perceptions regarding the world in which they live. If this is the case, and there is no reason to believe otherwise, then the systematic exclusion of women from a social category integrally tied to perhaps the most highly marketable traits imaginable in our increasingly wired and technology-rich world has ramifications far beyond inequality for inequality's sake. Women are underrepresented in nearly every branch of industry related to technology, computers, and the hard sciences (LaPonsie, 2012; Lee, 2002). While there exists no simple solution to this discrepancy, it is perhaps safe to assume that if television 


\section{ARE WE COOL YET?}

and films portrayed girls and women in these roles with greater regularity, the fields of science and technology would appear less foreign or inhospitable to women who might hope to pursue careers therein.

While this study focused the bulk of its analysis on disparities between male and female portrayals of nerds, these results also provide powerful evidence that identification as a nerd is racially coded as well. Of the 78 nerds observed in the course of analysis, all but six (7.7\%) were Caucasian; this disparity is greater even than that between male and female nerd portrayals, and holds similar cultural implications. As with women, racial minorities remain underrepresented in the fields of science and technology (Chang, Eagen, Lin, \& Hurtado, 2011; Lent, Lopez, Sheu, \& Lopez, 2011), a trend which can only be exacerbated by exclusion from such tech-savvy television portrayals as those presented by nerds. While there has been a small amount of scholarly inquiry conducted into the question of whether nerds, as a subculture, constitute a disruption of hegemonic White culture (Kendall, 2011; Quail, 2011), these studies provide no compelling reason to believe that such destabilization is taking place, at least not as pertaining to race. The significance of the mass media as a source of production and dissemination of sociocultural ideologies cannot be overstated, particularly with respect to issues of race, class, gender, and sexuality (Hall, 1995; hooks, 1995). The current study provides significant evidence that even as nerds have become a widely recognized and discussed (if not yet widely televised) social phenomenon, portrayals of nerds remain dictated by hegemonic associations of whiteness with giftedness (Staiger, 2004). The theory of media cultivation (Gerbner, 1998) dictates that television portrayals 
ARE WE COOL YET?

which are highly homogenous are likely, through repetition and over time, to impact viewers' perceptions and beliefs regarding the world they inhabit. Given this, the homogeneity of nerd representations in popular television (i.e., as being, almost without exception, white males) may serve, to some extent, to both construct and legitimize the underrepresentation of women and people of color in the fields of science and technology. This potentiality is exceptionally troubling, particularly in light of the fact that the racial and gender disparities observed in these fields show little sign of diminishment, even in recent years (Chang et al., 2011; LaPonsie, 2012; Lent et al., 2011).

To illustrate the significance of these implications, it is possible to further draw upon the works of the media scholars cited above in order to posit that the homogenous portrayal of nerds in popular television programs is likely to impact viewer perceptions and beliefs without conscious awareness of such effects on the part of audience members. Survey respondents frequently provided responses which were seemingly incongruous, with perceptions of nerds on television failing to align with perceptions of nerds in real life. The point which must be emphasized, however, is that in the vast majority of cases, when characters were considered nerds, they were also assessed more poorly in terms of physical attractiveness and social competence even when participants held positive feelings toward nerds in general. Perhaps there is a greater tolerance for these negative traits among those individuals who enjoy the company of nerds and who believe being a nerd is, at the least, not something of which one should be ashamed, or perhaps these television portrayals are simply crafted in such a way as to permit no contrary readings. 
ARE WE COOL YET?

What remains evident is that even among individuals who self-identify as holding positive views regarding nerds, physical unattractiveness and social incompetence have become such integral facets of what it means to be a nerd that it is difficult, if not impossible, to perceive a television character as a nerd without also ascribing these characteristics to that character.

It is also intriguing to note that in nearly all cases, those participants who were positively inclined toward nerds were concomitantly more likely to perceive characters as nerds than were those participants who possessed negative feelings toward nerds. On the one hand, this is perhaps to be expected. The principle of parasocial interaction (Conway \& Rubin, 1991; 2001) states that audience members construct interpersonal relationships with the characters and personalities they observe on television, and it stands to reason that such relationships are likely to foster feelings of familiarity and similarity. Similarly, assessments of characters' physical attractiveness were nearly always greater among those individuals with pro-nerd than with anti-nerd perceptions; in the case of social competence, however, the opposite was observed in regards to every one of the characters included in the questionnaire. While this finding seemingly flies in the face of reason, one possible explanation is that social incompetence has been emphasized more strongly within the mass media as a characteristic of nerds than has physical unattractiveness. If this is in fact the case, through the effects of cultivation (Gerbner, 1998; Gerbner \& Gross, 1976), those individuals who are more invested in the nerd subculture and who hold more positive views regarding nerds in general are resultantly more likely to self-select into exposure to nerd portrayals, with the result being that these 
ARE WE COOL YET?

individuals adhere more strongly to the idea that social incompetence is an inherent and fundamental prerequisite for identification as a nerd.

In sum, the results of this study provide compelling evidence that the subcultural identity of the nerd has been, and remains to the present day, less than inclusive of anyone other than white males. It seems likely that mass media characterizations of nerds are archetypes representative of extant social and cultural norms and values regarding gender, race, and the relationship thereto of technological and scientific expertise (Guzman \& Stanton, 2009). The social construction and delimitation of these fields as being strictly the purview of white males is clearly neither so recent nor so straightforward as to have originated with the representation of the stereotypical nerd in the mass media (Lee, 2002). Nonetheless, problematic trends have clearly been established in the portrayal of nerds on popular broadcast televisions shows, with such portrayals serving to both perpetuate and legitimize the archetype of the nerd - the possessor of those scientific and technological tools by which the doors to the future might be unlocked - as being, nearly without exception, white and male. This is not to say, of course, that on an individual level, individuals who self-identify as nerds are consciously aware of the exclusionary nature of this group; however, as has been emphatically illustrated in the course of the current study, the images of nerds presented in the mass media are far from heterogeneous.

Irrespective of the level of conscious awareness of this fact among nerds and nonnerds alike, significant evidence exists (e.g., Gerbner, 1998; Lippmann, 1922; McCombs \& Shaw, 1972) to suggest that consistent and unvaried media portrayals do indeed have 
ARE WE COOL YET?

the potential to impact viewers' beliefs and perceptions of the world in which they live. This dynamic is clearly illustrated in the data resultant from the current study in that survey respondents adhered to stereotypical ideas regarding nerds even as they simultaneously expressed tolerance, or even fondness, for this subcultural group. No shortage of attention has been devoted to the underrepresentation of women and racial minorities in the fields of science and technology (Chang et al., 2011; Lent et al., 2011), and the identity of the nerd holds remarkable potential for the rectification of these disparities. As nerds are currently conceptualized and portrayed in the mass media, however, such underrepresentation is likely to be exacerbated rather than mitigated, a trend which must unquestionably be corrected.

\section{Limitations and Future Research}

Although every attempt was made to be as comprehensive as possible in exploring popular television programming and public opinion and perception regarding nerds, this study is not without limitations. First, the sampling of television programs on the basis of Nielsen ratings was chosen in order, it is hoped, to examine the programs which are likely to have been viewed by the largest possible number of audience members. Given the current study's focus upon the sociocultural implications of television portrayals, breadth of audience exposure was of paramount importance in the selection of programs to be studied, and Nielsen ratings provided the most reliable means available of ascertaining such audience exposure. While the rationale for this sampling method is sound, it necessitated the exclusion of numerous programs, past and present, which have been previously identified as containing characters who exemplify many of 


\section{ARE WE COOL YET?}

the characteristics of nerds included in this study. The characters in shows which do not appeal sufficiently to the mainstream audience which provides the ratings necessary to reach the top five may, in fact, appeal more to niche audiences and subcultures such as nerds. If this is in fact the case, it follows that these characters may be considered more true to the self-perceptions held by those within this subculture. Thus, while the sample of programs included in the current study was perfectly suited to analyzing as influential a corpus of television as possible, future studies might draw upon less popular niche programs to add further nuance to the understanding of mass media portrayals of nerds.

Suggestions for future research projects which might serve this function include a quantitative content analysis, similar to that undertaken in the current study, but devoted strictly to television programs which are ascertained to include content and characters focusing primarily on nerds and nerd subculture. Examples of such shows, based on the articles from which the researcher drew television characters for this study's survey questionnaire, include Chuck, The Big Bang Theory, The Simpsons, and Freaks and Geeks. In addition to (or possibly prior to) further content analysis, the researcher recommends that qualitative inquiry be undertaken to assess the extent to which individuals who do and do not self-identify as nerds feel that various television programs accurately portray nerds and nerd subculture. Again, such inquiry would differ significantly from the study presented in this paper. Whereas the current study addresses broad and generalizable trends in the most popular broadcast television programs for the past twenty years, research such as that suggested here would be far less generalizable and would be primarily focused on ensuring that no audience perspectives were ignored. 


\section{ARE WE COOL YET?}

The results of such a research project, in addition to being highly informative and valuable in their own right, would serve to inform subsequent quantitative content analysis and survey-based studies.

Additionally, while numerous studies have addressed the implications of nerd identification, no researcher has yet undertaken a content analysis such as that included in the present study; thus, the operationalization of nerd characters for coding purposes is without precedent. As noted above, participant response to survey questions regarding perceptions of nerds provided a measure of external validity to this operationalization; nonetheless, it remains likely that adjustments should be made to account for changes in nerd characteristics over the years. Even if the central characteristics remain unchanged, simplification and standardization of coding procedures for nerd identification may result in more straightforward analysis for future researchers. In order to address this need, the researcher suggests further public opinion surveys to explore and confirm the validity of the construct established in the context of this study. Concurrently, a more comprehensive and varied questionnaire should be developed, with items assessed for compatibility and standardized measures consequentially established for each distinct aspect of nerd characterization.

Closely related to the last point is the trend, discussed earlier, of the "Hollywood Nerd" archetype which has grown increasingly prevalent in television and film (tvtropes.com, n.d.). The survey included in the current study focused upon characters identified as nerds by external sources; however, analysis of participant responses revealed that two of these characters, Willow Rosenberg and Chuck Bartowski, differed 
ARE WE COOL YET?

substantially from the other three characters. These two characters were consistently rated as more attractive and more socially competent than Milhouse van Houten, Steve Urkel, and Sheldon Cooper; concomitant with this assessment, participants were substantially less likely to identify Willow and Chuck as nerds than to make such assessments of Milhouse, Urkel, and Sheldon. To the best of the researcher's knowledge, there exist no studies to this point which have addressed the phenomenon of the Hollywood nerd; it seems highly probable, however, that such an analysis is overdue, and the inclusion of this archetype in the current study may have yielded interesting and enlightening results. Future research projects which might address this new trope in nerd portrayals include public opinion and factor analysis studies such as those outlined above, as well as qualitative studies to explore the extent to which "Hollywood nerd"-type characters are perceived as nerds by those within and without the subculture.

Lastly, and perhaps most importantly in regards to the analysis of television content regarding nerds, the scope of the current study included non-nerd characters only inasmuch as such characters were noted for numerical comparative purposes, with no information collected pertaining to demographic or study variables. The choice to exclude non-nerd characters from more comprehensive analysis was a conscious one, intended to ensure that the study sample could be as large as possible while remaining within the temporal and logistical constraints inherent in such a project. Nonetheless, the absence of detailed information regarding non-nerd characters renders any comparison (aside from pure frequency of appearance) between nerds and non-nerds impossible. In order to address this potential shortcoming, it is suggested that further content analysis be 
ARE WE COOL YET?

undertaken which encompasses a smaller sample of programs and episodes but includes the full coding of both nerd and non-nerd characters in order to permit comparison between groups. The current study has been intended to open the door to a much larger body of future scholarship; the suggestions outlined in this section merely scratch the surface of potential inquiry, and it is hoped that the findings and implications outlined above will guide and inspire as broad a range as possible of subsequent research.

\section{Conclusion}

This project represents both the culmination of a lifetime of experiences and observations by the researcher who was long considered himself a nerd, and a first step toward a more comprehensive understanding of the sociocultural implications of this subcultural identity. Since their inception as a nonsensical make-believe creature in the world of Dr. Seuss (1950), nerds have grown to become an immediately recognizable facet of the American sociocultural landscape. This is unquestionably due, at least in part, to the technological and computer-related expertise with which nerds have always been associated. To be a nerd once meant a life of ostracism and scorn by those within mainstream culture; however, as society places an ever-increasing premium on the very skills possessed by this subculture of former outcasts, nerds have begun to experience an unprecedented level of attention and interest. Numerous magazines, newspapers, and websites have begun to document the emergence of this remarkable individual, this lovably awkward genius in whose hands rest the tools by which the world of the future will be shaped. Nerds in modern-day America are recognized, celebrated, even emulated by the very individuals who, mere decades earlier, would likely have found their 
ARE WE COOL YET?

company abhorrent, or at least unnecessary. But what are the implications of this sudden shift in perceptions? How has the coming of the "Golden age of the nerd" (Ross, 2010) impacted those who are living through it?

We live in a moment in history at which access to technology, while unequally distributed across geographic, racial, and socioeconomic lines, is nevertheless greater than at any point before. Contemporaneous with such access, however, is an everincreasing reliance upon the technologies with which we are surrounded on a daily basis. As reliance on new media and technologies increases, many individuals find themselves growing in some ways removed from the social interactions which once were commonplace; face-to-face interaction may decrease, as, indeed, may one's level of comfort or competence regarding such interaction (McQuillen, 2003). Given the new communication patterns which have accompanied this fundamental shift in our sociocultural landscape, it is perhaps unsurprising that nerds, with their lack of social graces and substantial, even excessive, familiarity with technology and science, have begun to resonate so strongly with some part of our collective psyche. Mass media portrayals of nerds, and particularly those presented in television programming, provide viewers with points of commonality and identification on the basis of the tech reliance and social disconnectedness attendant to modern life. In addition to such identification, however, nerd characters on television serve to reassure viewers that even if they feel disconnected from other humans and overly connected to the technological prostheses upon which they rely so heavily, it could be worse, for at least they're not that far gone. It is this sometimes-discomfiting recognition of similarity which lies at the heart of what 
ARE WE COOL YET?

renders the nerd, particularly now, so relevant and enduring a character. Even as the audience laughs at such portrayals, that laughter is often tinged with self-reflexive nervousness, for the viewer recognizes that we are ourselves, in one way or another, all nerds as well.

As the past chapters have illustrated, the frequency of nerd portrayals in popular television has not undergone the dramatic increase which might be expected on the basis of the amount of media attention devoted to nerds. What can be said, however, is that nerds on television remain at least moderately incompetent, and although such portrayals have grown more physically attractive in recent years, viewers remain likely to perceive unattractiveness and social incompetence as characteristics of nerds. Above all else, and it is not possible to place too strong an emphasis on this point, nerds in popular television programs are overwhelmingly, almost ludicrously, portrayed as white males, with women and people of color remaining systematically excluded from representation within such roles. While the extent to which viewers internalize and act upon the cultural scripts produced by the mass media remains a subject of debate among communication scholars, the fact that effects of some sort exist is beyond contention. Consequentially, the conceptualization of technological and scientific expertise as strictly the purview of white males is problematic in the extreme. The current study is intended not merely as a corpus of (hopefully) interesting and enlightening facts and figures; rather, it is the researcher's hope that it will serve as a call to action, a stark portrait of the vast and inexcusable disparities in the portrayal of a subculture on the cutting edge of sociocultural progress. 


\section{ARE WE COOL YET?}

It is possible, although such speculation is likely overly optimistic, that the homogenous representation of nerds within the mass media is due to a lack of awareness regarding the significance of this social group. Perhaps those with the power to craft and disseminate media messages have not yet considered the possibility that one reason why nerds may have begun to resonate so strongly with an ever-broadening segment of American culture is due to their proficiency with the very technologies upon which the world of the future will, it is believed, be constructed. The identity of the nerd, entwined as it is with technological and scientific knowledge, holds enormous potential for the mitigation of racial and gender disparities in such fields. If nerd identification is portrayed in television (and elsewhere throughout the mass media) as open to all, without regard for sex or race, it is possible that slowly but surely, viewers of such portrayals will begin to see that they, too, can find a place if they desire one within these industries of the future. Without such equality, however, all the much-hyped progress of the nerd within mainstream culture is meaningless. It is not enough for Gilbert to get the girl; only when the girl is Gilbert, or a version thereof, can the nerds truly be triumphant, and the geeks inherit the earth. 
ARE WE COOL YET?

Table 1: Most-Viewed Scripted Broadcast Television Shows by Year

\begin{tabular}{|c|c|c|c|c|c|}
\hline Year & $\begin{array}{l}1^{\text {st }} \text { Most- } \\
\text { Viewed }\end{array}$ & $\begin{array}{l}2^{\text {nd }} \text { Most- } \\
\text { Viewed }\end{array}$ & $\begin{array}{l}3^{\text {rd }} \text { Most- } \\
\text { Viewed }\end{array}$ & $\begin{array}{l}4^{\text {th }} \text { Most- } \\
\text { Viewed }\end{array}$ & $\begin{array}{l}5^{\text {th }} \text { Most- } \\
\text { Viewed }\end{array}$ \\
\hline 1992 & Roseanne & $\begin{array}{l}\text { Murphy } \\
\text { Brown }\end{array}$ & Cheers & $\begin{array}{c}\text { Home } \\
\text { Improvement }\end{array}$ & $\begin{array}{l}\text { Designing } \\
\text { Women }\end{array}$ \\
\hline 1993 & Roseanne & $\begin{array}{c}\text { Home } \\
\text { Improvement }\end{array}$ & Murphy Brown & $\begin{array}{l}\text { Murder, She } \\
\text { Wrote }\end{array}$ & Coach \\
\hline 1994 & $\begin{array}{c}\text { Home } \\
\text { Improvement }\end{array}$ & Seinfeld & Roseanne & $\begin{array}{c}\text { These Friends } \\
\text { of Mine }\end{array}$ & $\begin{array}{c}\text { Grace Under } \\
\text { Fire }\end{array}$ \\
\hline 1995 & Seinfeld & ER & $\begin{array}{c}\text { Home } \\
\text { Improvement }\end{array}$ & $\begin{array}{c}\text { Grace Under } \\
\text { Fire }\end{array}$ & NYPD Blue \\
\hline 1996 & ER & Seinfeld & Friends & $\begin{array}{l}\text { Caroline in the } \\
\text { City }\end{array}$ & The Single Guy \\
\hline 1997 & ER & Seinfeld & Friends & $\begin{array}{l}\text { Suddenly } \\
\text { Susan }\end{array}$ & $\begin{array}{l}\text { The Naked } \\
\text { Truth }\end{array}$ \\
\hline 1998 & Seinfeld & ER & $\begin{array}{l}\text { Veronica's } \\
\text { Closet }\end{array}$ & Friends & Union Square \\
\hline 1999 & ER & Friends & Frasier & $\begin{array}{l}\text { Veronica's } \\
\text { Closet }\end{array}$ & Jesse \\
\hline 2000 & ER & Friends & Fraiser & The Practice & $\begin{array}{c}\text { Touched by an } \\
\text { Angel }\end{array}$ \\
\hline 2001 & ER & Friends & $\begin{array}{l}\text { Everybody } \\
\text { Loves } \\
\text { Raymond }\end{array}$ & The Practice & CSI \\
\hline 2002 & Friends & CSI & ER & $\begin{array}{l}\text { Everybody } \\
\text { Loves } \\
\text { Raymond }\end{array}$ & Law and Order \\
\hline 2003 & CSI & Friends & ER & $\begin{array}{l}\text { Everybody } \\
\text { Loves } \\
\text { Raymond }\end{array}$ & Law and Order \\
\hline 2004 & CSI & Friends & ER & CSI: Miami & $\begin{array}{l}\text { Everybody } \\
\text { Loves } \\
\text { Raymond }\end{array}$ \\
\hline 2005 & CSI & $\begin{array}{c}\text { Desperate } \\
\text { Housewives }\end{array}$ & CSI: Miami & $\begin{array}{l}\text { Without a } \\
\text { Trace }\end{array}$ & $\begin{array}{l}\text { Grey's } \\
\text { Anatomy }\end{array}$ \\
\hline 2006 & CSI & $\begin{array}{l}\text { Desperate } \\
\text { Housewives }\end{array}$ & $\begin{array}{l}\text { Grey's } \\
\text { Anatomy }\end{array}$ & $\begin{array}{l}\text { Without a } \\
\text { Trace }\end{array}$ & CSI: Miami \\
\hline 2007 & CSI & $\begin{array}{l}\text { Grey's } \\
\text { Anatomy }\end{array}$ & House, M.D. & $\begin{array}{c}\text { Desperate } \\
\text { Housewives }\end{array}$ & CSI: Miami \\
\hline 2008 & $\begin{array}{c}\text { Desperate } \\
\text { Housewives }\end{array}$ & House, M.D. & CSI & $\begin{array}{l}\text { Grey’s } \\
\text { Anatomy }\end{array}$ & NCIS \\
\hline 2009 & CSI & NCIS & The Mentalist & $\begin{array}{c}\text { Desperate } \\
\text { Housewives }\end{array}$ & $\begin{array}{c}\text { Two and a Half } \\
\text { Men }\end{array}$ \\
\hline 2010 & NCIS & The Mentalist & $\begin{array}{l}\text { NCIS: Los } \\
\text { Angeles }\end{array}$ & CSI & $\begin{array}{c}\text { Desperate } \\
\text { Housewives }\end{array}$ \\
\hline 2011 & NCIS & $\begin{array}{l}\text { Two and a } \\
\text { Half Men }\end{array}$ & $\begin{array}{l}\text { NCIS: Los } \\
\text { Angeles }\end{array}$ & $\begin{array}{c}\text { The Big Bang } \\
\text { Theory }\end{array}$ & Modern Family \\
\hline
\end{tabular}


ARE WE COOL YET?

Table 2: Television Programs Included in Study Sample

\begin{tabular}{|c|c|c|}
\hline Program Title & $\begin{array}{c}\text { \# of Years in } \\
\text { Top Five }\end{array}$ & $\begin{array}{c}\text { \# of Episodes } \\
\text { Included in Sample }\end{array}$ \\
\hline Big Bang Theory, The & 1 & 3 \\
\hline CSI: Crime Scene Investigators & 10 & 30 \\
\hline CSI: Miami & 4 & 12 \\
\hline Caroline in the City & 1 & 3 \\
\hline Cheers & 1 & 3 \\
\hline Coach & 1 & 3 \\
\hline Designing Women & 1 & 3 \\
\hline Desperate Housewives & 6 & 18 \\
\hline ER & 10 & 30 \\
\hline Everybody Loves Raymond & 4 & 12 \\
\hline Frasier & 2 & 6 \\
\hline Friends & 9 & 27 \\
\hline Grace Under Fire & 2 & 6 \\
\hline Grey's Anatomy & 4 & 12 \\
\hline Home Improvement & 4 & 12 \\
\hline House, M.D. & 2 & 6 \\
\hline Jesse & 1 & 3 \\
\hline Law and Order & 2 & 6 \\
\hline Mentalist, The & 2 & 6 \\
\hline Modern Family & 1 & 3 \\
\hline Murder, She Wrote & 1 & 3 \\
\hline Murphy Brown & 2 & 6 \\
\hline NCIS & 4 & 12 \\
\hline NCIS: Los Angeles & 2 & 6 \\
\hline NYPD Blue & 1 & 3 \\
\hline Naked Truth, The & 1 & 3 \\
\hline Practice, The & 2 & 6 \\
\hline Roseanne & 3 & 9 \\
\hline Seinfeld & 5 & 15 \\
\hline Single Guy, The & 1 & 3 \\
\hline Suddenly Susan & 1 & 3 \\
\hline These Friends of Mine (Ellen) & 1 & 3 \\
\hline Touched by an Angel & 1 & 3 \\
\hline Two and a Half Men & 2 & 6 \\
\hline Union Square & 1 & 3 \\
\hline Veronica's Closet & 2 & 6 \\
\hline Without a Trace & 2 & 6 \\
\hline
\end{tabular}


ARE WE COOL YET?

Table 3: Audience Perceptions of Popular Television Characters

\begin{tabular}{lccc}
\hline & $\begin{array}{c}\text { Nerd } \\
\text { Identification }\end{array}$ & $\begin{array}{c}\text { Social } \\
\text { Competence }\end{array}$ & $\begin{array}{c}\text { Physical } \\
\text { Attractiveness }\end{array}$ \\
\cline { 2 - 4 } \multicolumn{1}{c}{ Character Name and Program } & $M(S D)$ & $M(S D)$ & $M(S D)$ \\
\hline Chuck Bartowski, Chuck & $2.91(.98)$ & $3.08(.84)$ & $3.85(.89)$ \\
$\begin{array}{l}\text { Milhouse Van Houten, The Simpsons } \\
\begin{array}{l}\text { Dr. Sheldon Cooper, The Big Bang } \\
\text { Theory }\end{array}\end{array}$ & $3.62(1.10)$ & $2.48(.98)$ & $2.38(1.10)$ \\
$\begin{array}{l}\text { Steve Urkel, Family Matters } \\
\begin{array}{l}\text { Willow Rosenberg, Buffy the Vampire } \\
\text { Slayer }\end{array}\end{array}$ & $3.78(1.08)$ & $2.47(.99)$ & $3.15(1.06)$ \\
\hline
\end{tabular}

Table 4: Assessment of Nerdiness of Popular Television Characters by Proand Anti-Nerd Participants

\begin{tabular}{lcc}
\hline & Pro-Nerd & Anti-Nerd \\
\cline { 2 - 3 } \multicolumn{1}{c}{ Character Name and Program } & $M(S D)$ & $M(S D)$ \\
\hline Chuck Bartowski, Chuck** & $3.20(.92)$ & $2.68(.97)$ \\
Milhouse Van Houten, The Simpsons & $3.72(1.12)$ & $3.54(1.08)$ \\
Dr. Sheldon Cooper, The Big Bang Theory** & $3.96(1.02)$ & $3.44(1.08)$ \\
Steve Urkel, Family Matters* & $3.92(.99)$ & $3.67(1.00)$ \\
Willow Rosenberg, Buffy the Vampire Slayer*** & $3.36(1.06)$ & $2.70(1.05)$ \\
\hline$* p \leq .05 . * * * p \leq .001$. & &
\end{tabular}


ARE WE COOL YET?

Table 5: Differences in Mean Assessments of Popular Television Characters on the Basis of Nerd Identification by Pro-Nerd Respondents

\begin{tabular}{|c|c|c|}
\hline & $\begin{array}{c}\text { Character } \\
\text { Considered a Nerd }\end{array}$ & $\begin{array}{c}\text { Character Not } \\
\text { Considered a Nerd }\end{array}$ \\
\hline Character Name (Variable Name) & $M(S D)$ & $M(S D)$ \\
\hline $\begin{array}{l}\text { Chuck Bartowski (Physical } \\
\text { Attractiveness) }\end{array}$ & $3.83(.90)$ & $4.03(1.04)$ \\
\hline Chuck Bartowski (Social Competence)** & $2.84(.78)$ & $3.43(.82)$ \\
\hline Chuck Bartowski (Tech. Expertise) & $3.63(.76)$ & $3.71(.78)$ \\
\hline $\begin{array}{l}\text { Milhouse Van Houten (Physical } \\
\text { Attractiveness)* }\end{array}$ & $2.24(1.15)$ & $2.58(1.06)$ \\
\hline $\begin{array}{l}\text { Milhouse Van Houten (Social } \\
\text { Competence)** }\end{array}$ & $2.04(.83)$ & $2.89(.89)$ \\
\hline Milhouse Van Houten (Tech. Expertise)** & $3.60(1.16)$ & $2.61(1.06)$ \\
\hline $\begin{array}{l}\text { Dr. Sheldon Cooper (Physical } \\
\text { Attractiveness) }\end{array}$ & $3.28(1.12)$ & $3.25(.99)$ \\
\hline $\begin{array}{l}\text { Dr. Sheldon Cooper (Social } \\
\text { Competence)** }\end{array}$ & $1.88(.94)$ & $2.85(.68)$ \\
\hline Dr. Sheldon Cooper (Tech. Expertise) ${ }^{* *}$ & $4.55(.72)$ & $3.39(.91)$ \\
\hline Steve Urkel (Physical Attractiveness) & $2.37(1.08)$ & $2.44(1.07)$ \\
\hline Steve Urkel (Social Competence)** & $2.26(.90)$ & $2.88(.81)$ \\
\hline Steve Urkel (Tech. Expertise)** & $4.00(.91)$ & $2.70(1.01)$ \\
\hline $\begin{array}{l}\text { Willow Rosenberg (Physical } \\
\text { Attractiveness)** }\end{array}$ & $4.33(.81)$ & $3.73(1.06)$ \\
\hline $\begin{array}{l}\text { Willow Rosenberg (Social } \\
\text { Competence)** }\end{array}$ & $2.63(.98)$ & $3.19(.64)$ \\
\hline Willow Rosenberg (Tech. Expertise)** & $3.99(.80)$ & $3.30(.93)$ \\
\hline
\end{tabular}
$* p \leq .05 . * * p \leq .001$. 
ARE WE COOL YET?

Table 6: Differences in Mean Assessments of Popular Television Characters on the Basis of Nerd Identification by Anti-Nerd Respondents

\begin{tabular}{|c|c|c|}
\hline & $\begin{array}{c}\text { Character } \\
\text { Considered a Nerd }\end{array}$ & $\begin{array}{c}\text { Character Not } \\
\text { Considered a Nerd }\end{array}$ \\
\hline Character Name (Variable Name) & $M(S D)$ & $M(S D)$ \\
\hline $\begin{array}{l}\text { Chuck Bartowski (Physical } \\
\text { Attractiveness)** }\end{array}$ & $3.65(.93)$ & $4.09(.66)$ \\
\hline Chuck Bartowski (Social Competence)** & $3.01(.71)$ & $3.45(.97)$ \\
\hline Chuck Bartowski (Tech. Expertise) & $3.41(.76)$ & $3.51(.95)$ \\
\hline $\begin{array}{l}\text { Milhouse Van Houten (Physical } \\
\text { Attractiveness)* }\end{array}$ & $2.19(1.09)$ & $2.61(1.03)$ \\
\hline $\begin{array}{l}\text { Milhouse Van Houten (Social } \\
\text { Competence)** }\end{array}$ & $2.18(.89)$ & $3.11(.88)$ \\
\hline Milhouse Van Houten (Tech. Expertise)** & $3.19(1.17)$ & $2.64(.96)$ \\
\hline $\begin{array}{l}\text { Dr. Sheldon Cooper (Physical } \\
\text { Attractiveness)* }\end{array}$ & $2.86(1.08)$ & $3.22(.97)$ \\
\hline $\begin{array}{l}\text { Dr. Sheldon Cooper (Social } \\
\text { Competence)** }\end{array}$ & $2.13(.79)$ & $3.24(.79)$ \\
\hline Dr. Sheldon Cooper (Tech. Expertise) ${ }^{* *}$ & $4.15(.97)$ & $3.58(.80)$ \\
\hline Steve Urkel (Physical Attractiveness) ${ }^{* *}$ & $1.94(.80)$ & $2.61(.97)$ \\
\hline Steve Urkel (Social Competence) ${ }^{* *}$ & $2.39(.87)$ & $2.99(.74)$ \\
\hline Steve Urkel (Tech. Expertise)** & $3.62(.94)$ & $2.99(.82)$ \\
\hline $\begin{array}{l}\text { Willow Rosenberg (Physical } \\
\text { Attractiveness) }\end{array}$ & $3.81(1.02)$ & $3.91(1.02)$ \\
\hline $\begin{array}{l}\text { Willow Rosenberg (Social } \\
\text { Competence)** }\end{array}$ & $2.85(.97)$ & $3.39(.90)$ \\
\hline Willow Rosenberg (Tech. Expertise)* & $3.83(1.00)$ & $3.45(.88)$ \\
\hline
\end{tabular}
$* p \leq .01 . * * p \leq .001$. 
ARE WE COOL YET?

Table 7: Multiple Regression Analysis Summary for Predictors of Nerd Identification of Television Character Chuck Bartowski

\begin{tabular}{|c|c|c|c|c|c|}
\hline Variable & $B$ & $S E B$ & $\beta$ & $t$ & $p$ \\
\hline Age & -.007 & .007 & -.068 & -1.14 & .25 \\
\hline Sex & -.079 & .117 & -.039 & -.67 & .50 \\
\hline Race & -.059 & .039 & -.088 & -1.53 & .12 \\
\hline Household Income & .006 & .050 & .008 & .12 & .89 \\
\hline Weekly Internet Use & .052 & .050 & .062 & 1.03 & .30 \\
\hline Weekly Television Viewing & -.012 & .025 & -.027 & -.46 & .64 \\
\hline Perceived Homophily & -.061 & .057 & -.062 & -1.06 & .28 \\
\hline Perceived Physical Attractiveness & -.062 & .074 & -.053 & -.841 & .40 \\
\hline Perceived Engagingness & .087 & .069 & .079 & 1.26 & .20 \\
\hline $\begin{array}{l}\text { Perceived Social Competence } \\
\text { (Normal)** }\end{array}$ & -.278 & .080 & -.258 & -3.49 & .001 \\
\hline $\begin{array}{l}\text { Perceived Social Competence } \\
\text { (Intimate)* }\end{array}$ & -.215 & .079 & -.201 & -2.73 & .007 \\
\hline $\begin{array}{l}\text { Perceived Technological/Computer } \\
\text { Expertise* }\end{array}$ & .200 & .077 & .16 & 2.58 & .01 \\
\hline
\end{tabular}


ARE WE COOL YET?

Table 8: Multiple Regression Analysis Summary for Predictors of Nerd Identification of Television Character Milhouse Van Houten

\begin{tabular}{|c|c|c|c|c|c|}
\hline Variable & $B$ & $S E B$ & $\beta$ & $t$ & $p$ \\
\hline Age & -.002 & .006 & -.017 & -.31 & .75 \\
\hline Sex & -.011 & .111 & -.006 & -.10 & .91 \\
\hline Race & .026 & .037 & .037 & .69 & .49 \\
\hline Household Income & .065 & .049 & .075 & 1.34 & .18 \\
\hline Weekly Internet Use & .027 & .046 & .032 & .58 & .55 \\
\hline Weekly Television Viewing & -.011 & .023 & -.025 & -.47 & .63 \\
\hline Perceived Homophily & -.081 & .051 & -.090 & -1.58 & .11 \\
\hline Perceived Physical Attractiveness* & -.134 & .059 & -.138 & -2.26 & .02 \\
\hline Perceived Engagingness* & .121 & .052 & .136 & 2.34 & .02 \\
\hline Perceived Social Competence (Normal) & -.100 & .064 & -.106 & -1.56 & .11 \\
\hline $\begin{array}{l}\text { Perceived Social Competence } \\
\text { (Intimate)** }\end{array}$ & -.350 & .065 & -.360 & -5.34 & $<.001$ \\
\hline $\begin{array}{l}\text { Perceived Technological/Computer } \\
\text { Expertise** }\end{array}$ & .162 & .050 & .181 & 3.27 & .001 \\
\hline
\end{tabular}


ARE WE COOL YET?

Table 9: Multiple Regression Analysis Summary for Predictors of Nerd Identification of Television Character Dr. Sheldon Cooper

\begin{tabular}{|c|c|c|c|c|c|}
\hline Variable & $B$ & $S E B$ & $\beta$ & $t$ & $p$ \\
\hline Age & .005 & .006 & .043 & .88 & .37 \\
\hline Sex & -.016 & .105 & -.007 & -.15 & .88 \\
\hline Race & -.025 & .035 & -.033 & -.71 & .47 \\
\hline Household Income & .061 & .046 & .065 & 1.33 & .18 \\
\hline Weekly Internet Use* & .090 & .044 & .097 & 2.02 & .04 \\
\hline Weekly Television Viewing & -.013 & .022 & -.028 & -.57 & .56 \\
\hline Perceived Homophily & -.069 & .045 & -.075 & -1.52 & .12 \\
\hline Perceived Physical Attractiveness & -.071 & .052 & -.070 & -1.35 & .17 \\
\hline Perceived Engagingness* & .111 & .058 & .102 & 1.91 & .05 \\
\hline $\begin{array}{l}\text { Perceived Social Competence } \\
\text { (Normal)** }\end{array}$ & -.191 & .067 & -.194 & -2.86 & .005 \\
\hline $\begin{array}{l}\text { Perceived Social Competence } \\
\text { (Intimate) } \text { (I** }^{*}\end{array}$ & -.275 & .065 & -.277 & -4.25 & $<.001$ \\
\hline $\begin{array}{l}\text { Perceived Technological/Computer } \\
\text { Expertise*** }\end{array}$ & .317 & .065 & .264 & 4.86 & $<.001$ \\
\hline
\end{tabular}


ARE WE COOL YET?

Table 10: Multiple Regression Analysis Summary for Predictors of Nerd Identification of Television Character Steve Urkel

\begin{tabular}{|c|c|c|c|c|c|}
\hline Variable & $B$ & $S E B$ & $\beta$ & $t$ & $p$ \\
\hline Age & .006 & .006 & .050 & .99 & .32 \\
\hline Sex & -.172 & .097 & -.088 & -1.76 & .07 \\
\hline Race & -.011 & .033 & -.017 & -.32 & .74 \\
\hline Household Income* & .104 & .044 & .125 & 2.39 & .01 \\
\hline Weekly Internet Use & .025 & .041 & .031 & .60 & .54 \\
\hline Weekly Television Viewing & -.025 & .021 & -.062 & -1.21 & .22 \\
\hline Perceived Homophily & .031 & .050 & .032 & .61 & .54 \\
\hline Perceived Physical Attractiveness* & -.146 & .056 & -.145 & -2.59 & .01 \\
\hline Perceived Engagingness & .057 & .052 & .057 & 1.08 & .27 \\
\hline Perceived Social Competence (Normal) & -.029 & .061 & -.030 & -.47 & .63 \\
\hline $\begin{array}{l}\text { Perceived Social Competence } \\
\text { (Intimate)** }\end{array}$ & -.231 & .057 & -.243 & -4.02 & $<.001$ \\
\hline $\begin{array}{l}\text { Perceived Technological/Computer } \\
\text { Expertise** }\end{array}$ & .388 & .052 & .398 & 7.48 & $<.001$ \\
\hline
\end{tabular}


ARE WE COOL YET?

Table 11: Multiple Regression Analysis Summary for Predictors of Nerd Identification of Television Character Willow Rosenberg

\begin{tabular}{|c|c|c|c|c|c|}
\hline Variable & $B$ & $S E B$ & $\beta$ & $t$ & $p$ \\
\hline Age & -.006 & .007 & -.052 & -.92 & .35 \\
\hline Sex & -.201 & .121 & -.093 & -1.66 & .09 \\
\hline Race & -.022 & .041 & -.030 & -.54 & .58 \\
\hline Household Income & .065 & .052 & .070 & 1.24 & .21 \\
\hline Weekly Internet Use & .053 & .051 & .059 & 1.04 & .29 \\
\hline Weekly Television Viewing & -.034 & .026 & -.075 & -1.33 & .18 \\
\hline Perceived Homophily* & .111 & .056 & .111 & 1.97 & .04 \\
\hline Perceived Physical Attractiveness & -.055 & .076 & -.048 & -.72 & .47 \\
\hline Perceived Engagingness $* *$ & .180 & .071 & .153 & 2.51 & .01 \\
\hline $\begin{array}{l}\text { Perceived Social Competence } \\
\text { (Normal)** }\end{array}$ & -.202 & .074 & -.194 & -2.72 & .007 \\
\hline $\begin{array}{l}\text { Perceived Social Competence } \\
\text { (Intimate) } \text { ( }^{* * *}\end{array}$ & -.311 & .078 & -.284 & -3.96 & $<.001$ \\
\hline $\begin{array}{l}\text { Perceived Technological/Computer } \\
\text { Expertise* }\end{array}$ & .164 & .071 & .141 & 2.32 & .02 \\
\hline
\end{tabular}


ARE WE COOL YET?

Table 12: Assessment of Social Competence of Popular Television Characters by Pro- and Anti-Nerd Participants

\begin{tabular}{lcc}
\hline \multicolumn{1}{c}{ Character Name and Program } & Pro-Nerd & Anti-Nerd \\
\cline { 2 - 3 } & $M(S D)$ & $M(S D)$ \\
\hline Chuck Bartowski, Chuck** & $2.96(.82)$ & $3.18(.86)$ \\
Milhouse Van Houten, The Simpsons** & $2.33(.94)$ & $2.61(1.00)$ \\
Dr. Sheldon Cooper, The Big Bang Theory*** & $2.20(.97)$ & $2.69(.96)$ \\
Steve Urkel, Family Matters* & $2.42(.91)$ & $2.64(.88)$ \\
Willow Rosenberg, Buffy the Vampire Slayer** & $2.92(.87)$ & $3.25(.95)$ \\
\hline$* p \leq .05 . * * p \leq .01 . * * * p \leq .001$ & &
\end{tabular}

Table 13: Assessment of Physical Attractiveness of Popular Television Characters by Pro- and Anti-Nerd Participants

\begin{tabular}{lcc}
\hline & Pro-Nerd & Anti-Nerd \\
\cline { 2 - 3 } \multicolumn{1}{c}{ Character Name and Program } & $M(S D)$ & $M(S D)$ \\
\hline Chuck Bartowski, Chuck & $3.87(.92)$ & $3.83(.87)$ \\
Milhouse Van Houten, The Simpsons & $2.37(1.13)$ & $2.38(1.07)$ \\
Dr. Sheldon Cooper, The Big Bang Theory* & $3.29(1.07)$ & $3.04(1.04)$ \\
Steve Urkel, Family Matters & $2.38(1.08)$ & $2.20(.93)$ \\
Willow Rosenberg, Buffy the Vampire Slayer & $4.02(.99)$ & $3.88(1.01)$ \\
\hline$* p \leq .05$. & &
\end{tabular}


ARE WE COOL YET?

Table 14: Mean Assessment of Physical Attractiveness of Popular Television Characters by Pro-Nerd Respondents

\begin{tabular}{lcc}
\hline & Male & Female \\
\cline { 2 - 3 } \multicolumn{1}{c}{ Character Name and Program } & $M(S D)$ & $M(S D)$ \\
\hline Chuck Bartowski, Chuck & $3.75(.92)$ & $4.01(.91)$ \\
Milhouse Van Houten, The Simpsons** & $2.65(1.15)$ & $2.05(1.02)$ \\
Dr. Sheldon Cooper, The Big Bang Theory & $3.31(1.04)$ & $3.29(1.12)$ \\
Steve Urkel, Family Matters & $2.51(1.10)$ & $2.22(1.04)$ \\
Willow Rosenberg, Buffy the Vampire Slayer* & $3.88(1.11)$ & $4.18(.81)$ \\
\hline$* p \leq .05 . * * p \leq .001$. & &
\end{tabular}

Table 15: Mean Assessment of Physical Attractiveness of Popular Television Characters by Anti-Nerd Respondents

\begin{tabular}{lcc}
\hline & Male & Female \\
\cline { 2 - 3 } \multicolumn{1}{c}{ Character Name and Program } & $M(S D)$ & $M(S D)$ \\
\hline Chuck Bartowski, Chuck & $3.77(.96)$ & $3.91(.74)$ \\
Milhouse Van Houten, The Simpsons & $2.41(1.03)$ & $2.35(1.13)$ \\
Dr. Sheldon Cooper, The Big Bang Theory & $3.07(1.03)$ & $3.00(1.06)$ \\
Steve Urkel, Family Matters & $2.30(.93)$ & $2.07(.91)$ \\
Willow Rosenberg, Buffy the Vampire Slayer & $3.85(1.03)$ & $3.92(1.00)$ \\
\hline
\end{tabular}


ARE WE COOL YET?

Figure 1: Frequencies of Nerd and Non-Nerd Characters By Year, 1992-2011

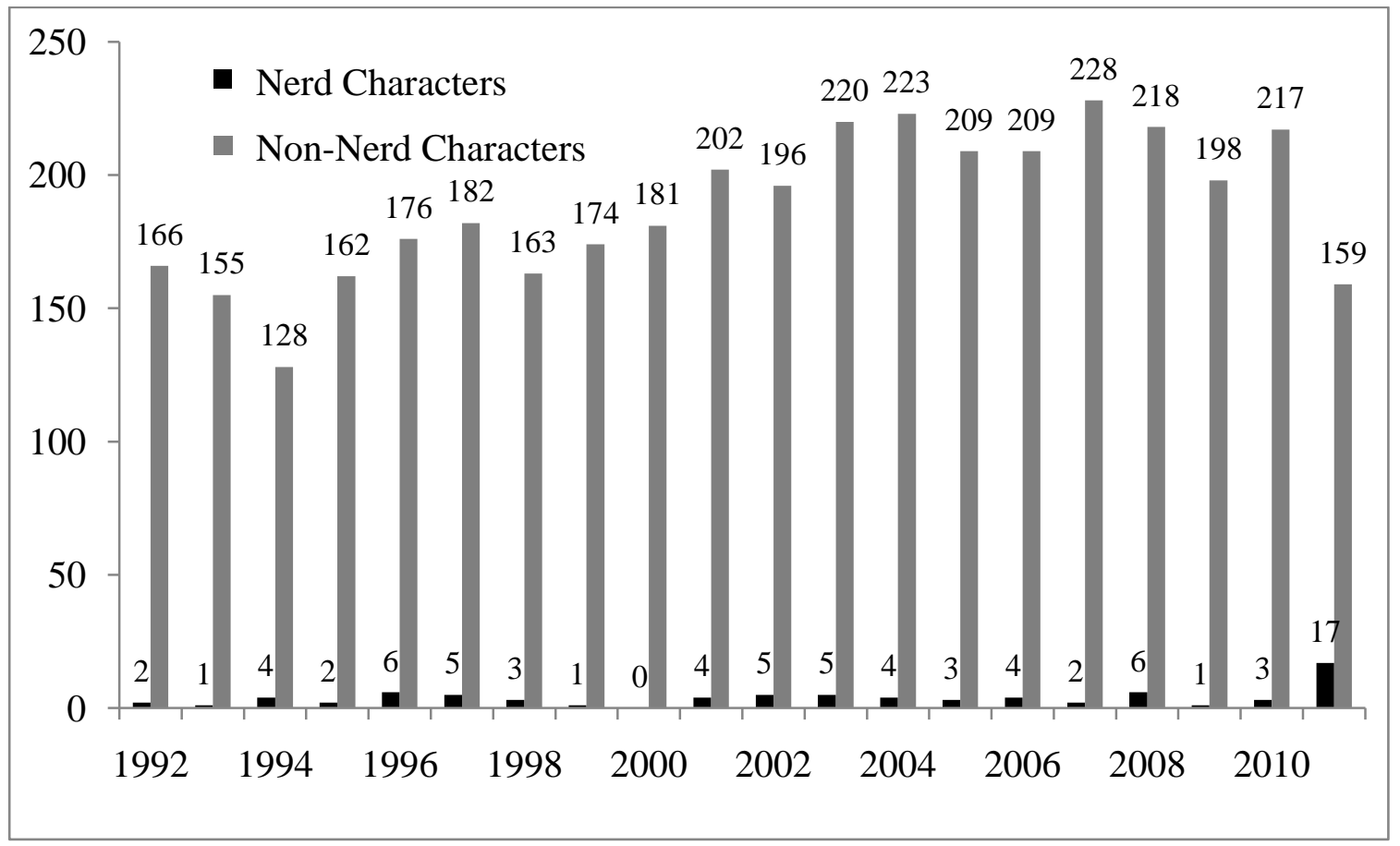

Figure 2: Frequencies of Nerd Characters in Comedic and Dramatic Programs By

Year, 1992-2011

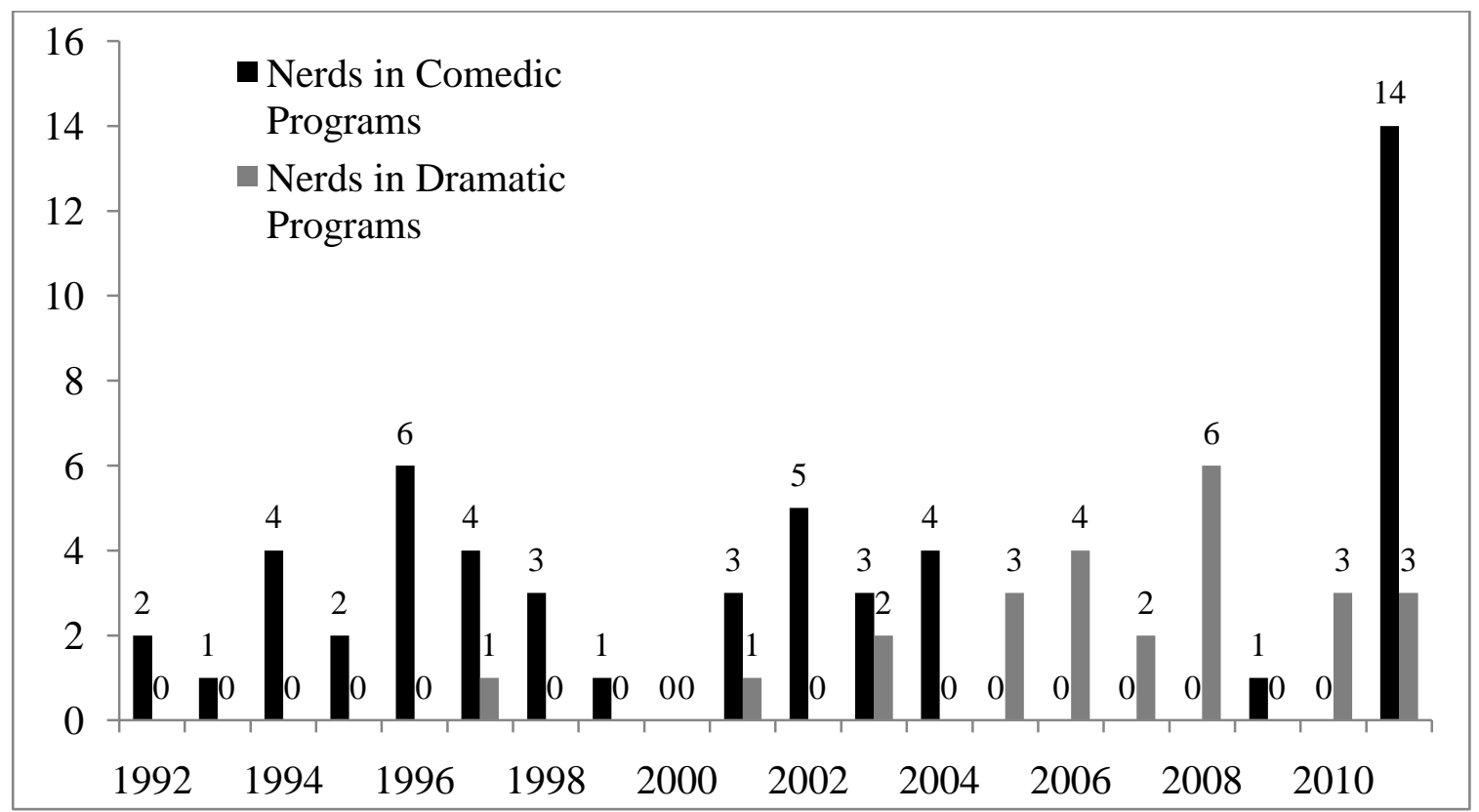




\section{ARE WE COOL YET?}

\section{References}

Alm, L. R., \& Davis, C. (1993). Agenda setting and acid precipitation in the United States. Environmental Management, 17(6), 807-816.

Anderegg, D. (2007). Nerds: Who they are and why we need more of them. New York: The Penguin Group.

Annese, S. (2004). Mediated identity in the parasocial interaction of TV. Identity, 4(4), 371-388.

Askehave, I., \& Nielsen, A. E. (2005). Digital genres: A challenge to traditional genre theory. Information Technology \& People, 18(2), 120-141.

Babbie, E. (2004). The practice of social research. Belmont, CA: Wadsworth Publishing Co.

Bakir, V. (2006). Policy agenda setting and risk communication. The Harvard International Journal of Press/politics, 11(3), 67-88.

Banet-Weiser, S., \& Portwood-Stacer, L. (2006). 'I just want to be me again!' Feminist Theory, 7(2), 255-272.

Baruh, L. (2009). Publicized intimacies on reality television: An analysis of voyeuristic content and its contribution to the appeal of reality programming. Journal of Broadcasting \& Electronic Media, 53(2), 190-210.

Bednarek, M. (2011). Expressivity and televisual characterization. Language and Literature, 20(1), 3-21. 
ARE WE COOL YET?

Buhrmester, M., Kwang, T., \& Gosling, S. D. (2011). Amazon's Mechanical Turk: A new source of inexpensive, yet high-quality, data?. Perspectives on Psychological Science, 6(1), 3-5.

Blickenstaff, J. C. (2005). Women and science careers: Leaky pipeline or gender filter?. Gender and Education, 17(4), 369-386.

Bratton, K., Haynie, K., \& Reingold, B. (2007). Agenda setting and African American women in state legislatures. Journal of Women, Politics \& Policy, 28, 71-96.

Bricken, R., \& Heiler, B. (2009, June 2). Pop culture's 10 greatest nerds. Topless Robot. Retrieved from http://www.toplessrobot.com/2009/06/pop_cultures_10_ greatest_nerds.php

Burrows, J. (2005). The origin of the nerd. Retrieved from http://eldacur.com/ brons/NerdCorner/nerd.html.

Butler, J. (1990). Gender trouble: Feminism and the subversion of identity. New York: Routledge.

Ceulemans, M., \& Fauconnier, G. (1979). Mass media: The image, role, and social conditions of women : a collection and analysis of research materials. Paris: Unesco.

Chang, M. J., Eagen, M. K., Lin, M. H., \& Hurtado, S. (2011). Considering the impact of racial stigmas and science identity: Persistence among biomedical and behavioral science aspirants. Journal of Higher Education, 82(5), 564-596.

Chen, X., \& French, D. C. (2008). Children's social competence in cultural context. Annual Review of Psychology, 59, 591-616. 


\section{ARE WE COOL YET?}

Chruscinski, D. (2008, June 20). Our favorite TV nerds. Starpulse.com. Retrieved from http://www.starpulse.com/news/index.php/2008/06/20/our_favorite_tv_nerds

Conway, J. C., \& Rubin, A. M. (1991). Psychological predictors of television viewing motivation. Communication Research, 18(4), 443-463.

Cross, T. (2005). Nerds \& geeks: Society's evolving stereotypes of our students with gifts and talents. The Gifted Child Today, 28(4), 26-27.

Dooling, R. (2008). Rapture for the geeks: When AI outsmarts IQ. New York: Harmony Books.

Duke, L. (2002). Get real!: Cultural relevance and resistance to the mediated feminine ideal. Psychology \& Marketing, 19(2), 211-233.

Erturk, Y. (2004). Considering the role of men in gender agenda setting: Conceptual and policy issues. Feminist Review, 2004, 78, 3-21.

Erwin, P. G. (1993). First names and perceptions of physical attractiveness. The Journal of Psychology, 127(6), 625-631.

Fan, J., Liu, F., Wu, J., \& Dai, W. (2004). Visual perception of female physical attractiveness. Proceedings: Biological Sciences, 271(1537), 347-352.

Field, A. E., Cheung, L., Wolf, A. M., Herzog, D. B., Gortmaker, S. L., \& Colditz, G. A. (1999). Exposure to mass media and weight concerns among girls. Pediatrics, 103(3), 54-60.

Fisherkeller, J. E. (1997). Everyday learning about identities among young adolescents in television culture. Anthropology \& Education Quarterly, 28(4), 467-92.

Fredrickson, B. L., Roberts, T., Noll, S. M., Quinn, D. M., \& Twenge, J. M. (1998). That 


\section{ARE WE COOL YET?}

swimsuit becomes you: Sex differences in self-objectification, restrained eating, and math performance. Journal of Personality and Social Psychology, 75(1), 269284.

Gallagher, M. (2004). Queer Eye for the heterosexual couple. Feminist Media Studies, $4(2), 223-226$.

Geek. (n.d.). In Oxford English Dictionary. Retrieved from http://dictionary.oed.com. Gerbner, G. (1998). Cultivation analysis: An overview. Mass Communication \& Society, $1(3 / 4), 175-194$.

Gerbner, G., \& Gross, L. (1976). Living with television: The violence profile. Journal of Communication, 26(2), 172-194.

Goldsborough, R. (2010, January 25). Technology today: Nerd, geek, dweeb, twerp - in computer lingo, which one are you? Retrieved from http://www.ccweek.com/news/templates/template.aspx?articleid=1629\&zoneid=3

Golebiowska, E. A. (1996). The "Pictures in our heads" and individual-targeted tolerance. Journal of Politics, 58(4), 1010-1034.

Grabe, S., Ward, L. M., \& Hyde, J. S. (2008). The role of the media in body image concerns among women: A meta-analysis of experimental and correlational studies. Psychological Bulletin, 134(3), 460-476.

Green, M. C., \& Brock, T. C. (2000). The role of transportation in the persuasiveness of public narratives. Journal of Personality and Social Psychology, 79(5), 701-21. 
ARE WE COOL YET?

Groesz, L. M., Levine, M. P., \& Murnen, S. K. (2002). The effect of experimental presentation of thin media images on body satisfaction: A meta-analytic review. International Journal of Eating Disorders, 31(1), 1-16.

Guzman, I. R., \& Stanton, J. M. (2009). IT occupational culture: The cultural fit and commitment of new information technologists. Information Technology \& People, 22(2), 157-187.

Haithman, D. (1991, January 04). Nerd power: Is uncool Urkel the '90s answer to the Fonz? The Los Angeles Times Online. Retrieved from http://articles.latimes.com/1991-01-04/entertainment/ca-7948_1_family-matters

Hall, S. (1973). Encoding and decoding in television discourse. Birmingham: CSS.

Hall, S. (1995). The whites of their eyes: Racist ideologies and the media. In G. Dines \& J. M. M. Humez (Eds.), Gender, race, and class in media: A text-reader (pp. 1822). Thousand Oaks, Calif: Sage.

Hammermeister, J., Brock, B., Winterstein, D., \& Page, R. (2005). Life without TV? Cultivation theory and psychosocial health characteristics of television-free individuals and their television-viewing counterparts. Health Communication, 17, 3, 253-264.

Hart, P., Nisbet, E., \& Shanahan, J. (2011). Environmental values and the social amplification of risk: An examination of how environmental values and media use influence predispositions for public engagement in wildlife management decision making. Society \& Natural Resources, 24(3), 276-291.

Hebdige, D. (1979). Subculture: The meaning of style. London: Methuen. 


\section{ARE WE COOL YET?}

Holmstrom, A. J. (2004).The effects of the media on body image: A meta-analysis. Journal of Broadcasting \& Electronic Media, 48(2), 196-217.

hooks, b. (1995). Madonna: Plantation mistress or soul sister? In G. Dines \& J. M. M. Humez (Eds.), Gender, race, and class in media: A text-reader (pp. 28-32). Thousand Oaks, Calif: Sage.

Jackson, S. (1998). Theorising gender and sexuality. In Jackson, S. \& Jones, J. (1998). Contemporary feminist theories (pp.131-146). New York: New York University Press.

Jansen, A. (2011, October 10). Romancing the nerds. The West Australian. Retrieved from http://au.news.yahoo.com/thewest/entertainment/a//television/10434395/romancing-the-nerds/

Kaufman, M. (1993). Cracking the armour: Power, pain and the lives of men. Toronto, Ontario, Canada: Viking.

Kendall, L. (1999). Nerd nation: Images of nerds in US popular culture. International Journal of Cultural Studies, 2(2), 260-283.

Kendall, L. (2000). "Oh no! I'm a nerd!": Hegemonic masculinity on an online forum. Gender and Society, 14(2), 256-274.

Kendall, L. (2011). "White and nerdy": Computers, race, and the nerd stereotype. Journal of Popular Culture, 44(3), 505-524.

Kiousis, S., \& Wu, X. (2008). International agenda-building and agenda-setting: Exploring the influence of public relations counsel on US news media and public 


\section{ARE WE COOL YET?}

perceptions of foreign nations. International Communication Gazette, 70(1), 5875.

Koesten, J. (2004). Family communication patterns, sex of subject, and communication competence. Communication Monographs, 71(2), 226-244.

Krippendorff, K. (2004). Content analysis: An introduction to its methodology. Thousand Oaks, Calif: Sage.

Lakoff, R. T. (1983). Doubletalk: Sexism in tech talk. In Zimmerman, J. (1983). The technological woman (pp. 38-43). New York: Praeger Publishers.

Landolt, P., Goldring, L., \& Bernhard, J. (2011). Agenda setting and immigrant politics: The case of Latin Americans in Toronto. American Behavioral Scientist, 55(9), $1235-1266$.

LaPonsie, M. (2012). Are women opting out of science careers? Online-Education.net. Retrieved from http://www.online-education.net/articles/science/are-womenopting-out-of-science-careers.html.

Lee, J. D. (2002). More than ability: Gender and personal relationships influence science and technology involvement. Sociology of Education, 75(4), 349-373.

Lent, R. W., Lopez, F. G., Sheu, H. B., \& Lopez, A. M. (2011). Social cognitive predictors of the interests and choices of computing majors: Applicability to underrepresented students. Journal of Vocational Behavior, 78(2), 184-192.

Lerner, R. M., Orlos, J. B., \& Knapp, J. R. (1976). Physical attractiveness, physical effectiveness, and self-concept in late adolescents. Adolescence, 11(43), 313-326. 


\section{ARE WE COOL YET?}

Liberman, M. (2011, August 27). Nerds, alpha and otherwise. Language Log. Retrieved from http://languagelog.ldc.upenn.edu/nll/?p=3391.

Lippmann, W. (1922). Public opinion. New York: Harcourt, Brace and Company.

Lombard, M., Snyder-Duch, J., \& Bracken, C. C. (2010, June 1). Practical resources for assessing and reporting intercoder reliability in content analysis research projects. Retrieved from http://matthewlombard.com/reliability/

Long, M., Steinke, J., Applegate, B., Lapinski, M. K., Johnson, M. J., \& Ghosh, S. (2010). Portrayals of male and female scientists in television programs popular among middle school-age children. Science Communication, 32(3), 356-382.

Longo, L. C., \& Ashmore, R. D. (1995). The looks-personality relationship: Global selforientations as shared precursors of subjective physical attractiveness and selfascribed traits. Journal of Applied Social Psychology, 25(5), 371-398.

Losh, S. (2010). Stereotypes about scientists over time among US adults: 1983 and 2001. Public Understanding of Science, 19(3), 372-382.

Lund, T. (2005). The qualitative-quantitative distinction: Some comments. Scandinavian Journal of Educational Research, 49, 2, 115-132.

Margolis, J., \& Fisher, A. (2002). Unlocking the clubhouse: Women in computing. Cambridge, Mass: MIT Press.

Maxim, P. S. (1999). Quantitative research methods in the social sciences. New York: Oxford University Press.

McArthur, J. A. (2009). Digital subculture. Journal of Communication Inquiry, 33(1), 5870. 
ARE WE COOL YET?

McComas, K. (2006). Defining moments in risk communication research: 1996-2005. Journal of Health Communication, 11(1), 75-91.

McCombs, M. E., \& Shaw, D. L. (1972). The agenda-setting function of mass media. Public Opinion Quarterly, 36(2), 176-187.

McCroskey, J. C., Richmond, V. P., \& Daly, J. A. (1975). The development of a measure of perceived homophily in interpersonal communication. Human Communication Research, 1, 323-332.

McLuhan, M., \& Fiore, Q. (1967). The medium is the message. New York: Bantam Books.

McQuillen, J. S. (2003). The influence of technology on the initiation of interpersonal relationships. Education, 123(3), 616-623.

Melosi, M. V. (2000). Environmental justice, political agenda setting, and the myths of history. Journal of Policy History, 12(1), 43-71.

Meng, H. (2008). Social script theory and cross-cultural communication. Intercultural Communication Studies, 17(1), 132-138.

Miller, M. (2008). Branding Miss G: Third wave feminists and the media. Toronto: Sumach Press.

Mittell, J. (2001). A cultural approach to television genre theory. Cinema Journal, 40(3), 3-24.

Montemurro, B. (2003). Not a laughing matter: Sexual harassment as "material" on workplace-based situation comedies. Sex Roles, 48, 433-445.

Moorti, S., \& Ross, K. (2004). Reality television. Feminist Media Studies, 4(2), 203-205. 
ARE WE COOL YET?

Morgan, M., Leggett, S., \& Shanahan, J. (1999). Television and family values: Was Dan Quayle right?. Mass Communication \& Society, 2, 47-63.

Nerd. (2012). In Merriam-Webster Unabridged Dictionary. Retrieved from http://www.merriam-webster.com/dictionary/nerd.

Neuendorf, K. A., Gore, T. D., Dalessandro, A., Janstova, P., \& Snyder-Suhy, S. (2010). Shaken and stirred: A content analysis of women's portrayals in James Bond films. Sex Roles, 62, 747-761.

Newsweek. (1951, October 8). Jelly tot, square bear-man!. Newsweek, 28-29.

Olson, B., \& Douglas, W. (1997). The family on television: Evaluation of gender roles in situation comedy. Sex Roles: a Journal of Research, 36, 409-427.

Pappademas, A. (2011, March 18). Hollywood's leading geek. The New York Times. Retrieved from www.nytimes.com/2011/03/20/magazine/mag-20Snyder-t.html.

Patterson, F. K. (1956). Adult role in adolescent subculture innovation: A case study. The Journal of Educational Sociology, 30(2), 58-74.

Pralle, S. B. (2009). Agenda-setting and climate change. Environmental Politics, 18(5), 781-799.

Quail, C. (2011). Nerds, geeks, and the hip/square dialectic in contemporary television. Television and New Media, 12(5), 460-482.

Rinaldi, C. M., Kates, A. D., \& Welton, C. (2008). Understanding students' interactions: Why varied social tasks matter. Emotional \& Behavioural Difficulties, 13, (2), $127-140$. 


\section{ARE WE COOL YET?}

Robbins, A. (2011). The geeks shall inherit the Earth: Popularity, quirk theory, and why outsiders thrive after high school. New York: Hyperion.

Rohner, J. C., \& Rasmussen, A. (2011). Physical attractiveness stereotype and memory. Scandinavian Journal of Psychology, 52(4), 309.

Ross, D. (2010, May 25). The golden age of the nerd. Retrieved from http://www.libertasfilmmagazine.com/the-golden-age-of-the-nerd/

Rubington, E. (1958). The chronic drunkenness offender. The Annals of the American Academy of Political and Social Science, 315(1), 65-72.

Schacht, R. (2005). Female and male perceptions of attractiveness: What is attractive and why?. DigitalCommons@University of Nebraska - Lincoln.

Schiappa, E., Gregg, P. B., \& Hewes, D. E. (2005). The parasocial contact hypothesis. Communication Monographs, 72(1), 92-115.

Schiappa, E., Hewes, D., \& Gregg, P. (2006). Can one TV show make a difference? Will \& Grace and the parasocial contact hypothesis. Journal of Homosexuality, 51(4), $15-37$.

Seuss, Dr., \& Random House (Firm). (1950). If I ran the zoo. New York: Random House. Shaw, A. (2010). What is video game culture? Cultural studies and game studies. Games and Culture, 5(4), 403-424.

Smiler, A. (2006). Living the image: A quantitative approach to delineating masculinities. Sex Roles, 55, 9-10.

Smythe, D. (1954). Reality as presented by television. Public Opinion Quarterly, 18(2), 143-156. 


\section{ARE WE COOL YET?}

Spertus, E. (1998). Ways that males and females are treated differently. Retrieved from http://people.mills.edu/spertus/Gender/pap/node7.html.

Staiger, A. (2004). Whiteness as giftedness: Racial formation at an urban high school. Social Problems, 51(2), 161-181.

Starcke, A. M. (2000). Not so "geeky" anymore. Techniques: Connecting Education and Careers, 75(7), 18-21.

Steinke, J. (2005). Cultural representations of gender and science. Science Communication, 27(1), 27-63.

Stice, J. (2011, August 27). 8 of the biggest sitcom nerds of all-time. Guyism.com. Retrived from http://guyism.com/lifestyle/the-8-biggest-sitcom-nerds-of-alltime.html

Stoilescu, D., \& Egodawatte, G. (2010). Gender differences in the use of computers, programming, and peer interactions in computer science classrooms. Computer Science Education, 20(4), 283-300.

Strelitz, L. N. (2003). Media consumption and identity formation: The case of the "homeland" viewers. Communication Abstracts, 26(2), 155-298.

Tedesco, J. (2005). Intercandidate agenda setting in the 2004 Democratic Presidential primary. American Behavioral Scientist, 49(1), 92-113.

The Digital Revolution: Once the privilege of a technical elite, computers and other digital technologies are rapidly becoming a common - and powerful - part of our professional and personal lives. (1998). Epri Journal, 23(1), 26-35. 
ARE WE COOL YET?

Thompson, M. A., \& Gray, J. J. (1995). Development and validation of a new bodyimage assessment scale. Journal of Personality Assessment, 64(2), 258-69.

Thornham, S. (1998). Feminist media and film theory. In S. Jackson \& J. Jones (Eds.), Contemporary feminist theories (pp.131-146). New York: New York University Press.

Tsai, W. S. (2010). Family man in advertising? A content analysis of male domesticity and fatherhood in Taiwanese commercials. Asian Journal of Communication, 20(4), 423-439.

tvtropes.org. (n.d.) Hollywood nerd. Retrived from http://tvtropes.org/pmwiki/pmwiki.php/Main/HollywoodNerd

Varma, R. (2000). Women in computing: The role of geek culture. Science As Culture, 16, 359-376.

Waggoner, C. E. (2004). Disciplining female sexuality in Survivor. Feminist Media Studies, 4(2), 217-220.

Ward, L. M., \& Harrison, K. (2005). The impact of media use on girls' beliefs about gender roles, their bodies, and sexual relationships: A research synthesis. In E. Cole \& J. H. Daniel (Eds.), Featuring females: Feminist analyses of media (pp. 323). Washington, DC: American Psychological Association.

Wiederman, M. (2005). The gendered nature of sexual scripts. The Family Journal, 13(4), 496-502. 


\section{ARE WE COOL YET?}

White, S., Brown, N. J., \& Ginsburg, S. (1999). Diversity of body types in network television programming: A content analysis. Communication Research Reports, 16(4), 386-392.

Wrench, J., \& McCroskey, J. (2001). A temperamental understanding of humor communication and exhilaratability. Communication Quarterly, 49(2), 142-159.

Xinsheng, L., Lindquist, E., \& Vedlitz, A. (2011). Explaining media and congressional attention to global climate change, 1969-2005: An empirical test of agenda-setting theory. Political Research Quarterly, 64(2), 405-419

Yinger, J. M. (1960). Contraculture and subculture. American Sociological Review, $25(5), 625-635$.

Younker, E. (2011, May 5). Study: Women still underrepresented in math, science fields. The Joplin Globe. Retrieved from http://www.joplinglobe.com/healthandfamily/x2023604081/Study-Women-stillunderrepresented-in-math-science-fields.

Zimmer, B. (2011, August 28). Birth of the nerd. The Boston. Retrieved from http://articles.boston.com/2011-08-28/bostonglobe/29939041_1_nerd-prideetymology-theories. 


\section{ARE WE COOL YET?}

\section{Appendix A: Codebook}

Overview of General Coding Procedures

1) Observe all characters in each episode in order to determine classification as a nerd or non-nerd.

2) Once nerd characters have been identified, code each for only those demographic variables which are evident through viewing of all sampled episodes in which that character appears.

3) Simultaneously, code all nerd characters for physical attractiveness and social competence based on the measures described below.

1. Nerd Identification (NERD):

Character is identified by self or others as a "nerd" or "geek"

Yes $=1$

$\mathrm{No}=0$

$\underline{\text { (In normal social interaction) }}$

Character stutters

Yes $=1$

No $=0$

Character appears unable to speak

Yes $=1$

No $=0$

Character engages in inappropriate speech or conversation 


\section{ARE WE COOL YET?}

Yes $=1$

$\mathrm{No}=0$

Character ignores other individuals

Yes $=1$

$\mathrm{No}=0$

Character appears nonplussed by behavior of others

Yes $=1$

$\mathrm{No}=0$

$\underline{\text { (In intimate romantic or sexual interaction) }}$

Character stutters

Yes $=1$

No $=0$

Character appears unable to speak to a potential partner

Yes $=1$

No $=0$

Character appears nonplussed by behavior of a potential partner

Yes $=1$

$\mathrm{No}=0$

Character or other(s) imply poor sexual performance on character's part

Yes $=1$

No $=0$

Character retreats from romantic or sexual interaction 


\section{ARE WE COOL YET?}

Yes $=1$

No $=0$

Character is either identified as a "nerd" or "geek," or she or he exhibits a minimum of three of the other characteristics outlined above:

Yes $=1$

No $=0$

\section{Coding Procedures}

1) Coders will observe each character and determine nerd identification based, whenever possible, upon statements made by that character or by others in reference to that character. If such identification is made, regardless of the presence or absence of other indicators, that character will be considered a nerd.

2) In cases in which such identification is not made, coders will determine nerd identification based upon the observation of each character's behaviors and the extent to which the five behaviors outlined above are present or absent.

3) Each character must be observed as exhibiting a minimum of three of these behaviors in order to be considered a nerd.

\section{Social Competence (SOCLCOMP):}

Ratio-level measurement; each instance in which another character exhibits a reaction which indicates the perception of inappropriate behavior, it will be coded as +1 , with a lower score on the resultant additive index reflecting a higher level of social competence. 


\section{ARE WE COOL YET?}

\section{Coding Procedures}

1) Coders will observe each nerd character's interactions with other nerd and nonnerd characters, paying particular attention to the reactions and responses of those others.

2) Each time another character reacts or responds to the nerd character in question in such a way as to clearly indicate their perception that the character in question has behaved or acted inappropriately, coders will consider this one "instance."

3) The total number of instances for each character will be considered a negative indicator of that character's level of social competence.

3. Physical attractiveness (PHYSATTR):

Extremely unattractive $=1$

Unattractive $=2$

Average $=3$

Attractive $=4$

Extremely attractive $=5$

Coding Procedures

1) Coders will observe each nerd character and determine physical attractiveness based upon physical appearance and the likelihood that that character will be considered physically or sexually appealing to others. 


\section{ARE WE COOL YET?}

2) Markers of physical attractiveness, as outlined by Schacht (2005), include facial and body symmetry, proportionality of facial features, and youthfulness of facial and body features.

3) Examples of, in order: Male (1), Female (1); Male (5), Female (5)
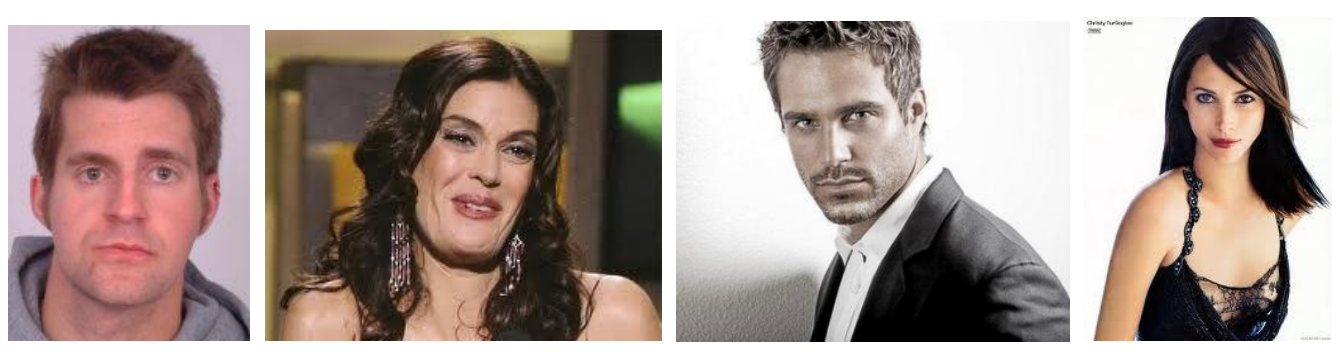

4. Body Shape (BODYSHAPE):

1) Coders will observe each nerd character and determine body shape as operationalized through the use of Thompson and Gray's (1995) nine-point measurement tool (see below).

2) In cases in which body shape is unclear, character will be coded as " 0 ". 


\section{ARE WE COOL YET?}
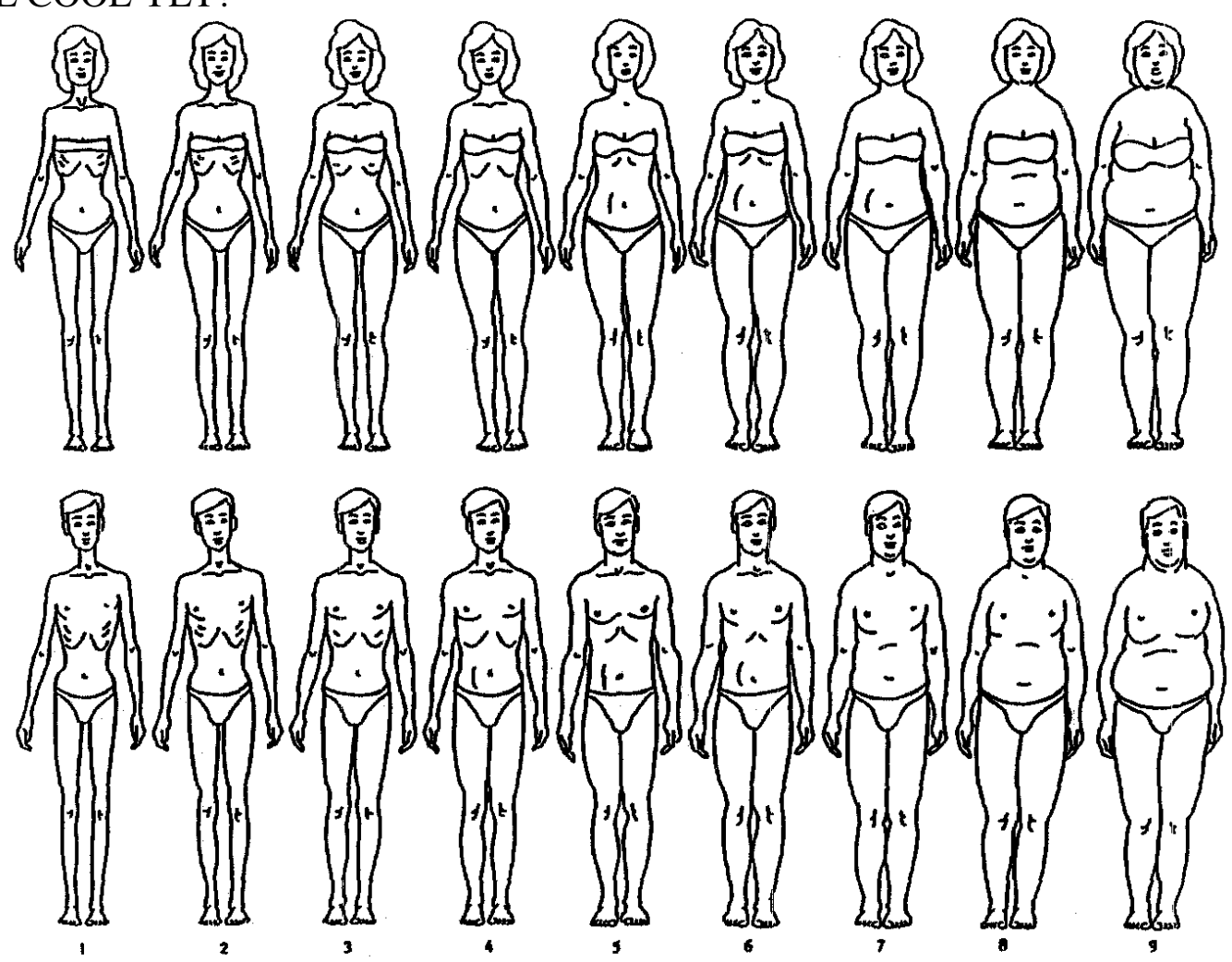

FIGURE 1 Contour Drawing Rating Scale.

3) $\operatorname{Sex}(\mathrm{SEX})$ :

Male $=1$

Female $=2$

Unable to determine $=0$

Coding Procedures

1) Coders will observe each nerd character and determine sex based upon physical appearance and pronouns used in reference to that character.

2) In cases in which sex is unclear, character will be coded as " 0 ".

4) Estimated age group (AGE): 


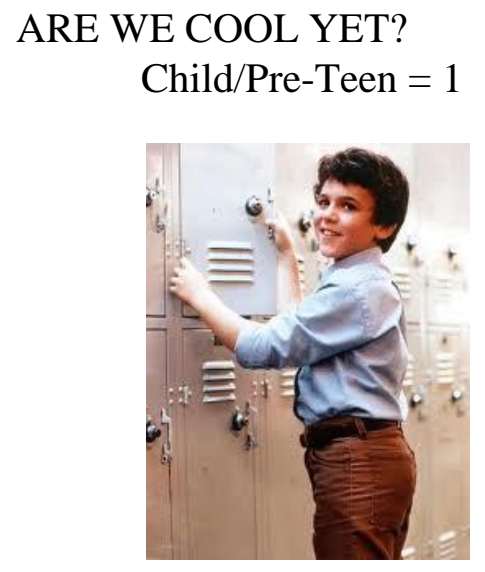

Teenager $=2$

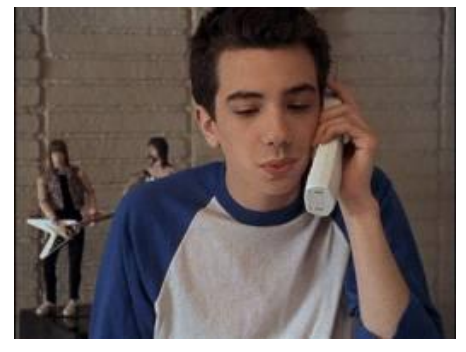

Young Adult = 3

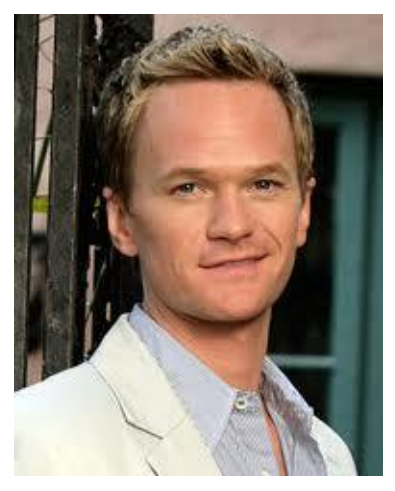

Middle-Aged = 4 


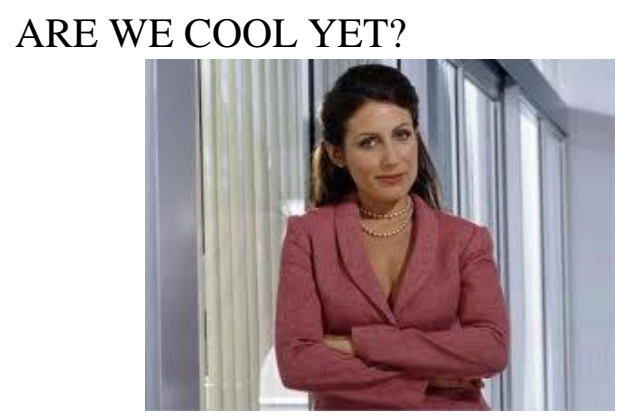

Elderly $=5$

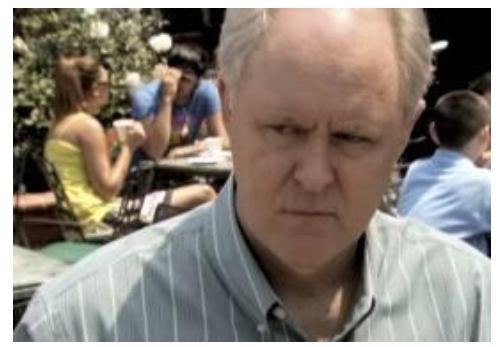

Unable to determine $=0$

Coding Procedures

1) Coders will observe each nerd character and determine age group based primarily upon manifest physical attributes (hair color, wrinkles, etc.) as well as activities and lifestyle (grade school student, college student, parent, etc.).

2) In cases in which age group is unclear, character will be coded as " 0 ".

5) Socioeconomic Status (SES):

Exhibits clear financial need $=1$

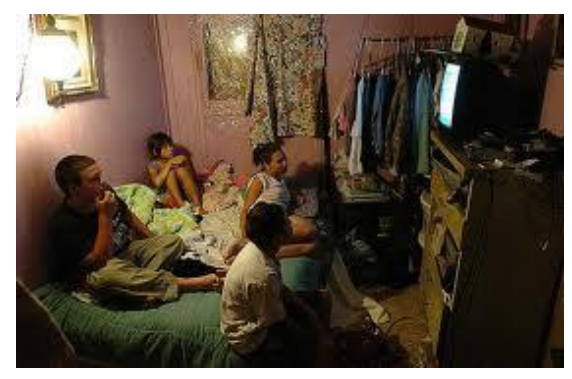

Exhibits no clear financial need and no exceptional affluence $=2$ 


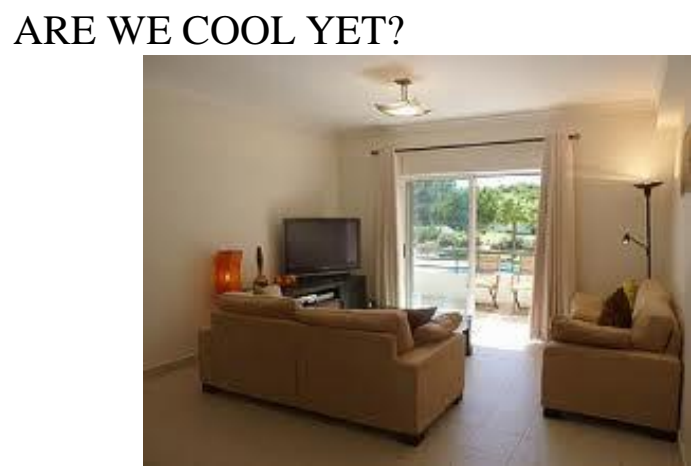

Exhibits clear exceptional affluence $=3$

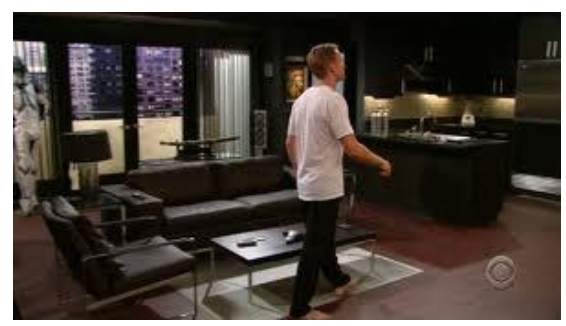

Unable to determine $=0$

Coding Procedures

1) Coders will observe each nerd character and determine SES based upon the conditions in which that character lives, their possessions, their state and manner of dress, and the extent to which they discuss money as a cause for concern in their lives.

2) In rare cases, other characters may ascribe SES to a given character; in cases such as this, the ascribed SES will be selected by coders unless such ascription is clearly contraindicated by observation of that character's lifestyle.

3) In cases in which SES is unclear, character will be coded as " 0 ".

6) Race (RACE):

African-American $/$ Black $=1$ 


\section{ARE WE COOL YET?}

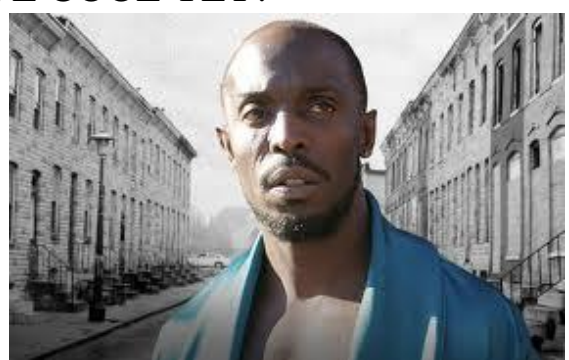

American Indian/Alaskan Native $=2$

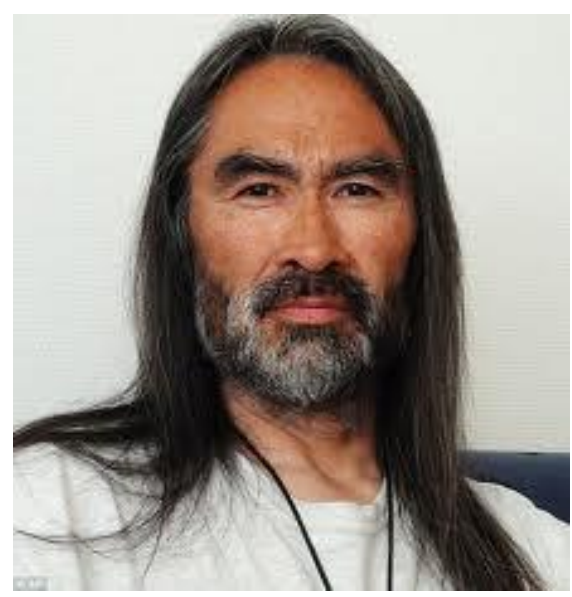

Asian/Pacific Islander $=3$ 


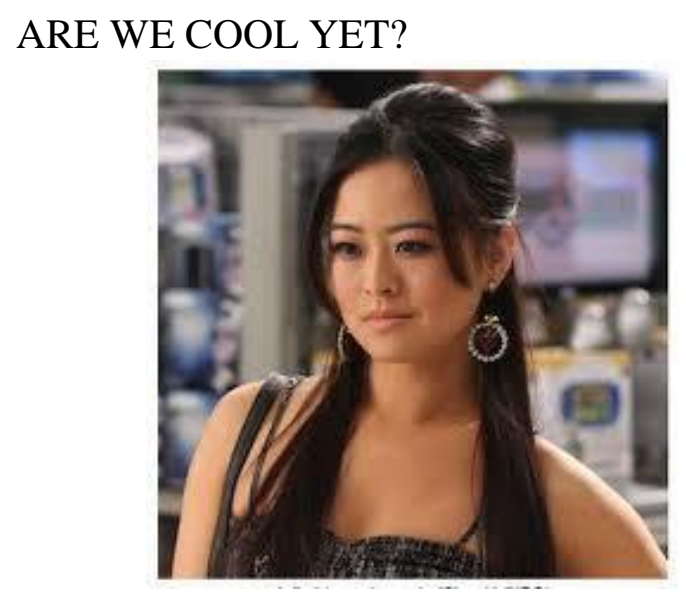

Caucasian $=4$

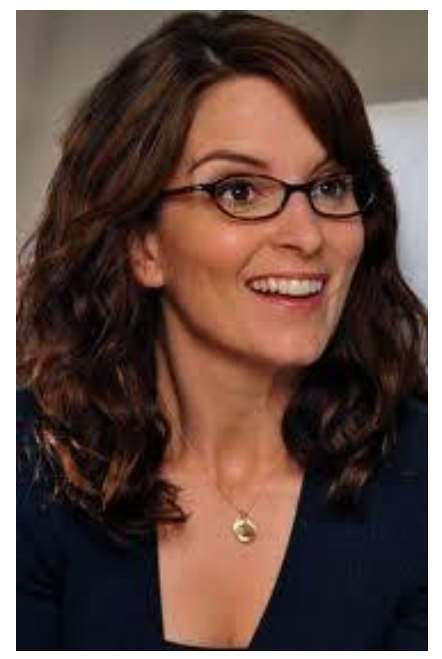

Hispanic or Latino/a $=5$

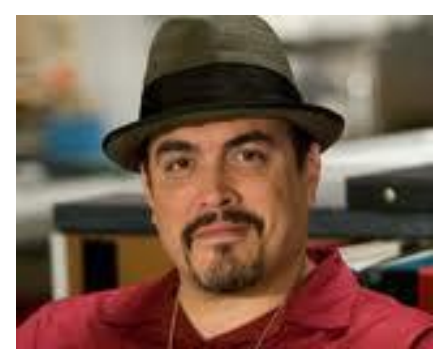

Indian $=6$ 
ARE WE COOL YET?

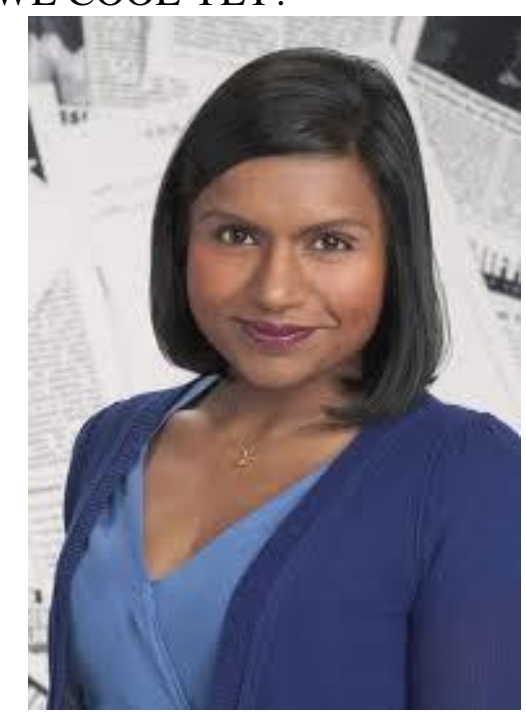

Two or more races $=7$

Other $=8$

Unspecified/Unable to determine $=0$

\section{Coding Procedures}

1) Coders will observe each nerd character and determine race based, whenever possible, upon statements made by that character or by others in reference to that character.

2) In cases in which such statements are unavailable, coders will determine race based upon skin tone and physical features, when such features are sufficiently unambiguous as to provide a reliable coding.

3) In cases in which race is unclear, character will be coded as " 0 ". 


\section{Appendix B: Survey Questionnaire}

Thank you for taking the time to complete this questionnaire! Thank you for participating in this study. Our purpose is to examine public opinion and perception regarding nerds and geeks.

Participants in this study should be 18 years of age or older. Completion of this survey is completely voluntary and your responses to the demographics section, in addition to all subsequent questions, will remain anonymous. You may quit at any time and you may refuse to answer any questions on the survey.

There are only minimal risks associated with participating in this study, as you might feel uncomfortable making assessments of television characters and individuals in reality. Overall, risks associated are less than one generally experiences in everyday life.

Thank you very much for your participation!

1. Please list what you feel are the top five characteristics of a nerd.

A. People often have opinions about nerds. For the following fourteen items, please select the response which best describes your opinion regarding each statement.

\section{General Perceptions of Nerds}

1. My friends probably consider me a nerd.

Strongly Disagree / Disagree / Neutral / Agree / Strongly Agree

2. When I think of a nerd, I think of someone with whom I would like to spend time.

Strongly Disagree / Disagree / Neutral / Agree / Strongly Agree

3. I think of myself as a nerd.

Strongly Disagree / Disagree / Neutral / Agree / Strongly Agree

4. I think being considered a nerd is a good thing.

Strongly Disagree / Disagree / Neutral / Agree / Strongly Agree 


\section{ARE WE COOL YET?}

5. I think being a nerd is a good thing.

Strongly Disagree / Disagree / Neutral / Agree / Strongly Agree

\section{Perceived Nerd Intelligence}

6. In general, I believe most nerds are intelligent.

Strongly Disagree / Disagree / Neutral / Agree / Strongly Agree Perceived Nerd Science/Tech Expertise

7. Most nerds know a lot about computers, science, or both.

Strongly Disagree / Disagree / Neutral / Agree / Strongly Agree

Perceived Nerd Engagingness

8. I find most nerds engaging.

Strongly Disagree / Disagree / Neutral / Agree / Strongly Agree

\section{Perceived Nerd Social Competence}

9. Most nerds don't know how to act around other people.

Strongly Disagree / Disagree / Neutral / Agree / Strongly Agree

10. Most nerds are uncomfortable with or confused by romance.

Strongly Disagree / Disagree / Neutral / Agree / Strongly Agree

\section{Perceived Nerd Physical Attractiveness}

11. I could imagine being physically attracted to a nerd.

Strongly Disagree / Disagree / Neutral / Agree / Strongly Agree

12. If someone's a nerd, they can't be handsome or beautiful.

Strongly Disagree / Disagree / Neutral / Agree / Strongly Agree

13. In general, I believe most nerds are physically attractive. 


\section{ARE WE COOL YET?}

Strongly Disagree / Disagree / Neutral / Agree / Strongly Agree Perceived Nerd Sex

14. It seems to me that most nerds are:

Women

Men

It doesn't seem like they're one more than the other 


\section{ARE WE COOL YET?}

A. In the following section, you will be asked questions about four characters from popular television shows. Please indicate first whether you recognize each character, and then answer the questions for each character.

For each item, please select the response which best describes your opinion.

1. Steve Urkel, Family Matters.

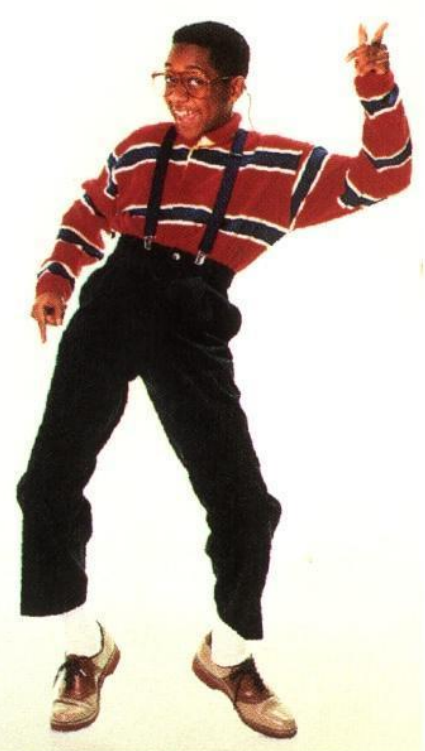

a. Are you familiar with this character?

Yes / No

b. I find this character engaging.

Strongly Disagree / Disagree / Neutral / Agree / Strongly Agree

c. This character seems physically attractive.

Strongly Disagree / Disagree / Neutral / Agree / Strongly Agree

d. This character seems to know a good deal about computers, science, or both.

Strongly Disagree / Disagree / Neutral / Agree / Strongly Agree

e. This character is often awkward or uncomfortable in his normal interaction with others.

Strongly Disagree / Disagree / Neutral / Agree / Strongly Agree

f. This character is often awkward or uncomfortable in his intimate or romantic interaction with others. 


\section{ARE WE COOL YET?}

Strongly Disagree / Disagree / Neutral / Agree / Strongly Agree

g. I would consider this character a nerd.

Strongly Disagree / Disagree / Neutral / Agree / Strongly Agree

For the next eight items, please indicate your feelings about each statement. Select the number that best represents your feelings. Numbers "1" and "7" indicate a very strong feeling. Numbers "2" and "6" indicate a strong feeling. Numbers " 3 " and "5" indicate a fairly weakfeeling. Number "4" indicates you are undecided or don't know. Please work quickly. There are no wrong answers.

h. This character:

1 - Doesn't think like me / 2 / 3 / 4 / 5 / 6 / 7 - Thinks like me

i. This character:

1 - Is from a social class similar to mine / 2 / 3 / 4 / 5 / 6 / 7 - Is from a social class different from mine

j. This character:

1 - Behaves like me / 2 / 3 / 4 / 5 / 6 / 7 - Doesn't behave like me

k. This character:

1 - Is from an economic situation / 2 / 3 / 4 / 5 / 6 / 7 - Is from an economic different from mine situation like mine.

1. This character:

1 - Is similar to me / 2 / 3/4 / 5/6/7 - Is different from me

m. This character:

1 - Has social status like mine / 2 / 3 / 4 / 5 / 6 / 7 - Has social status different from mine

n. This character:

1 - Is unlike me / 2/3/4/5/6/7 - Is like me

o. This character:

Has a background different from mine / 2 / 3 / 4 / 5 / 6 / 7 - Has a background similar to mine 
ARE WE COOL YET?

2. Sheldon Cooper, The Big Bang Theory

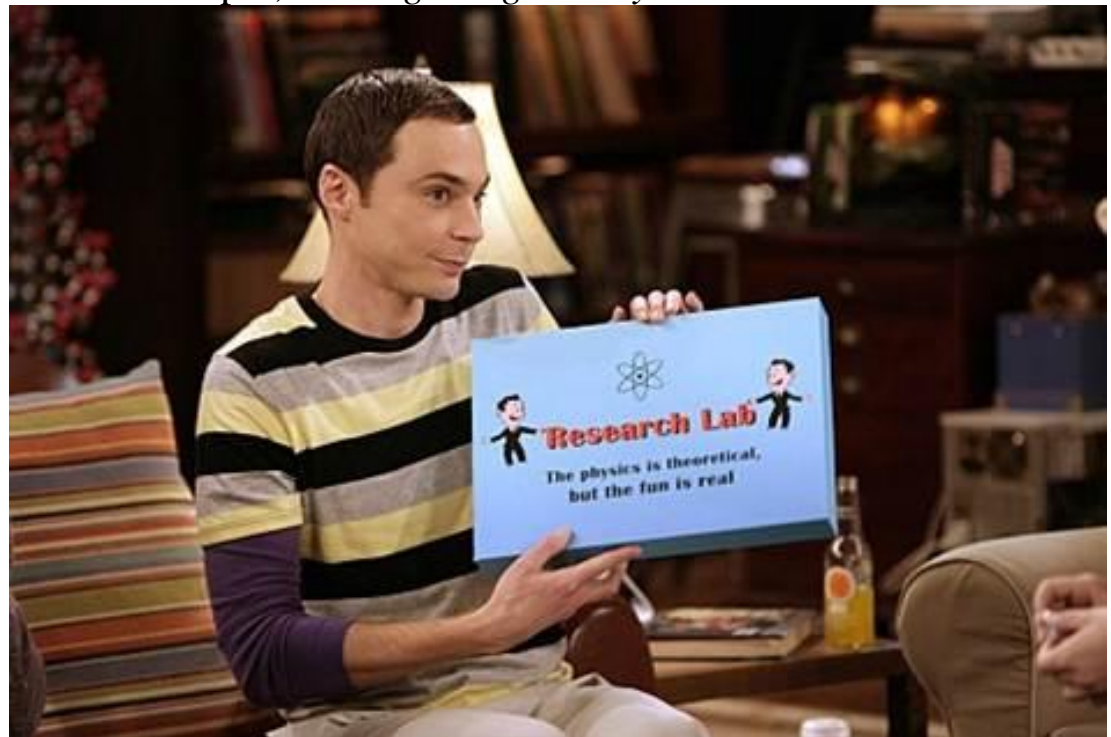

a. Are you familiar with this character?

Yes / No

b. I find this character engaging.

Strongly Disagree / Disagree / Neutral / Agree / Strongly Agree

c. This character seems physically attractive.

Strongly Disagree / Disagree / Neutral / Agree / Strongly Agree

d. This character seems to know a good deal about technology, science, or both.

Strongly Disagree / Disagree / Neutral / Agree / Strongly Agree

e. This character seems is often awkward or uncomfortable in his normal interaction with others.

Strongly Disagree / Disagree / Neutral / Agree / Strongly Agree

f. This character is often awkward or uncomfortable in his intimate or romantic interaction with others.

Strongly Disagree / Disagree / Neutral / Agree / Strongly Agree

g. I would consider this character a nerd. 


\section{ARE WE COOL YET?}

Strongly Disagree / Disagree / Neutral / Agree / Strongly Agree

For the next eight items, please indicate your feelings about each statement. Select the number that best represents your feelings. Numbers "1" and "7" indicate a very strong feeling. Numbers "2" and "6" indicate a strong feeling. Numbers "3" and "5" indicate a fairly weak feeling. Number "4" indicates you are undecided or don't know. Please work quickly. There are no wrong answers.

h. This character:

1 - Doesn't think like me / 2 / 3 / 4 / 5 / 6/ 7 - Thinks like me

i. This character:

1 - Is from a social class similar to mine / 2 / 3 / 4 / 5 / 6/ 7 - Is from a social class different from mine

j. This character:

1 - Behaves like me / 2 / 3 / 4 / 5 / 6 / 7 - Doesn't behave like me

k. This character:

1 - Is from an economic situation / 2 / 3 / 4 / 5 / 6 / 7 - Is from an economic different from mine situation like mine.

1. This character:

1 - Is similar to me / 2 / 3 / 4 / 5 / 6/ 7 - Is different from me

m. This character:

1 - Has social status like mine / 2 / 3 / 4 / 5 / 6 / 7 - Has social status different

n. This character: from mine

1 - Is unlike me / 2 / 3 / 4 / 5/ 6/7 - Is like me

o. This character:

Has a background different from mine / 2 / 3 / 4 / 5 / 6 / 7 - Has a background similar to mine 


\section{ARE WE COOL YET?}

3. Chuck Bartowski, Chuck

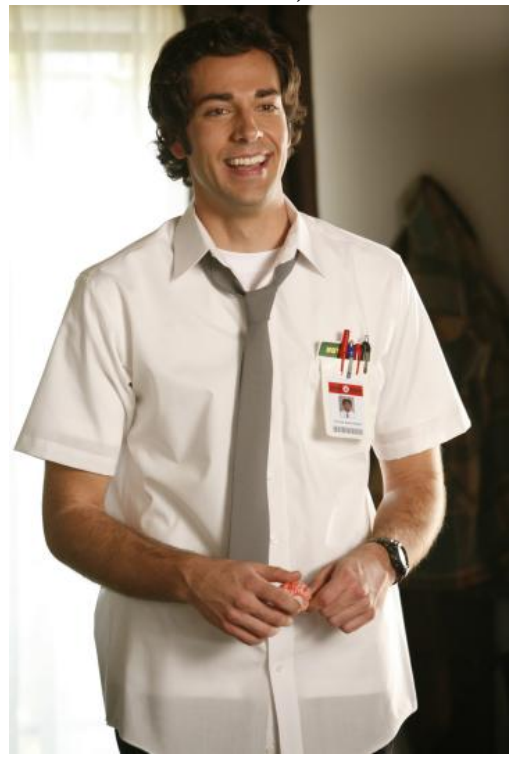

a. Are you familiar with this character?

Yes / No

b. I find this character engaging.

Strongly Disagree / Disagree / Neutral / Agree / Strongly Agree

c. This character seems physically attractive.

Strongly Disagree / Disagree / Neutral / Agree / Strongly Agree

d. This character seems to know a good deal about technology, science, or both.

Strongly Disagree / Disagree / Neutral / Agree / Strongly Agree

e. This character is often awkward or uncomfortable in his normal interaction with others.

Strongly Disagree / Disagree / Neutral / Agree / Strongly Agree

f. This character seems is often awkward or uncomfortable in his intimate or romantic interaction with others.

Strongly Disagree / Disagree / Neutral / Agree / Strongly Agree

g. I would consider this character a nerd. 


\section{ARE WE COOL YET?}

Strongly Disagree / Disagree / Neutral / Agree / Strongly Agree

For the next eight items, please indicate your feelings about each statement. Select the number that best represents your feelings. Numbers "1" and "7" indicate a very strong feeling. Numbers "2" and "6" indicate a strong feeling. Numbers "3" and "5" indicate a fairly weakfeeling. Number "4" indicates you are undecided or don't know. Please work quickly. There are no wrong answers.

h. This character:

1 - Doesn't think like me / 2 / 3 / 4 / 5 / 6 / 7 - Thinks like me

i. This character:

1 - Is from a social class similar to mine / 2 / 3 / 4 / 5 / 6/ 7 - Is from a social class different from mine

j. This character:

1 - Behaves like me / 2 / 3 / 4 / 5 / 6 / 7 - Doesn't behave like me

k. This character:

1 - Is from an economic situation / 2 / 3 / 4 / 5 / 6 / 7 - Is from an economic different from mine situation like mine.

1. This character:

1 - Is similar to me / 2 / 3 / 4 / 5 / 6/ 7 - Is different from me

m. This character:

1 - Has social status like mine / 2 / 3 / 4 / 5 / 6 / 7 - Has social status different

n. This character:

from mine

1 - Is unlike me / 2 / 3 / 4 / 5 / 6/7 - Is like me

o. This character:

Has a background different from mine / 2 / 3 / 4 / 5 / 6 / 7 - Has a background similar to mine 


\section{ARE WE COOL YET?}

4. Willow Rosenberg, Buffy the Vampire Slayer

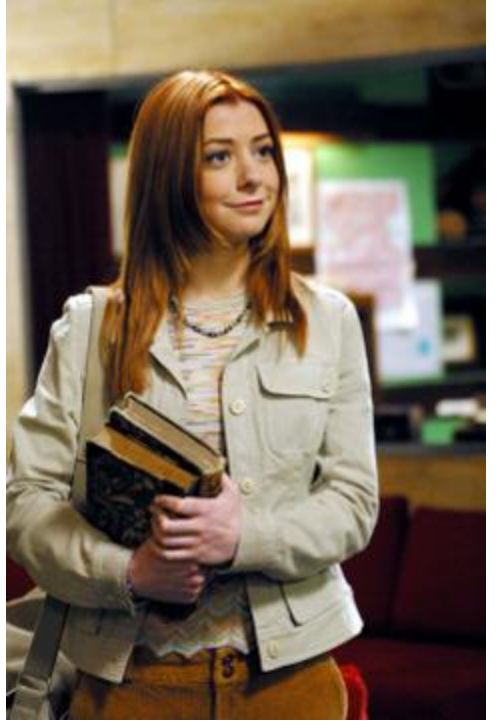

a. Are you familiar with this character?

Yes / No

b. I find this character engaging.

Strongly Disagree / Disagree / Neutral / Agree / Strongly Agree

c. This character seems physically attractive.

Strongly Disagree / Disagree / Neutral / Agree / Strongly Agree

d. This character seems to know a good deal about technology, science, or both.

Strongly Disagree / Disagree / Neutral / Agree / Strongly Agree

e. This character is often awkward or uncomfortable in her normal interaction with others.

Strongly Disagree / Disagree / Neutral / Agree / Strongly Agree

f. This character is often awkward or uncomfortable in her intimate or romantic interaction with others.

Strongly Disagree / Disagree / Neutral / Agree / Strongly Agree

g. I would consider this character a nerd. 


\section{ARE WE COOL YET?}

Strongly Disagree / Disagree / Neutral / Agree / Strongly Agree

For the next eight items, please indicate your feelings about each statement. Select the number that best represents your feelings. Numbers "1" and "7" indicate a very strong feeling. Numbers "2" and "6" indicate a strong feeling. Numbers "3" and "5" indicate a fairly weakfeeling. Number "4" indicates you are undecided or don't know. Please work quickly. There are no wrong answers.

h. This character:

1 - Doesn't think like me / 2 / 3 / 4 / 5 / 6/ 7 - Thinks like me

i. This character:

1 - Is from a social class similar to mine / 2 / 3 / 4 / 5 / 6 / 7 - Is from a social class different from mine

j. This character:

1 - Behaves like me / 2 / 3 / 4 / 5 / 6 / 7 - Doesn't behave like me

k. This character:

1 - Is from an economic situation / 2 / 3 / 4 / 5 / 6 / 7 - Is from an economic different from mine situation like mine.

1. This character:

1 - Is similar to me / 2 / 3 / 4 / 5 / 6/ 7 - Is different from me

m. This character:

1 - Has social status like mine / 2 / 3 / 4 / 5 / 6 / 7 - Has social status different

n. This character:

from mine

1 - Is unlike me / 2 / 3 / 4 / 5 / 6/7 - Is like me

o. This character:

Has a background different from mine / 2 / 3 / 4 / 5 / 6 / 7 - Has a background similar to mine 


\section{ARE WE COOL YET?}

5. Milhouse Van Houten, The Simpsons

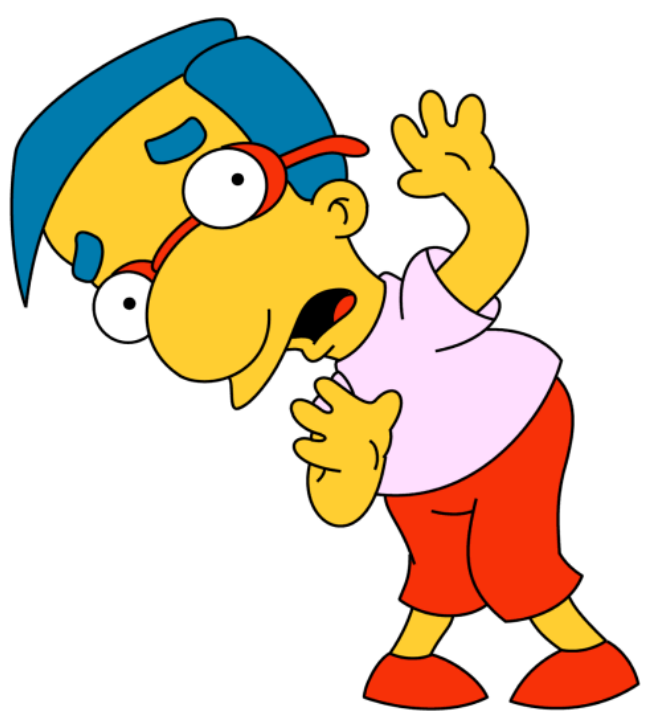

a. Are you familiar with this character?

Yes / No

b. I find this character engaging.

Strongly Disagree / Disagree / Neutral / Agree / Strongly Agree

c. This character seems physically attractive.

Strongly Disagree / Disagree / Neutral / Agree / Strongly Agree

d. This character seems to know a good deal about computers, science, or both.

Strongly Disagree / Disagree / Neutral / Agree / Strongly Agree

e. This character is often awkward or uncomfortable in his normal interaction with others.

Strongly Disagree / Disagree / Neutral / Agree / Strongly Agree

f. This character is often awkward or uncomfortable in his intimate or romantic interaction with others.

Strongly Disagree / Disagree / Neutral / Agree / Strongly Agree

g. I would consider this character a nerd. 


\section{ARE WE COOL YET?}

Strongly Disagree / Disagree / Neutral / Agree / Strongly Agree

For the next eight items, please indicate your feelings about each statement. Select the number that best represents your feelings. Numbers "1" and "7" indicate a very strong feeling. Numbers "2" and "6" indicate a strong feeling. Numbers "3" and "5" indicate a fairly weakfeeling. Number "4" indicates you are undecided or don't know. Please work quickly. There are no wrong answers.

h. This character:

1 - Doesn't think like me / 2 / 3 / 4 / 5 / 6/ 7 - Thinks like me

i. This character:

1 - Is from a social class similar to mine / 2 / 3 / 4 / 5 / 6 / 7 - Is from a social class different from mine

j. This character:

1 - Behaves like me / 2 / 3 / 4 / 5 / 6 / 7 - Doesn't behave like me

k. This character:

1 - Is from an economic situation / 2 / 3 / 4 / 5 / 6/7 - Is from an economic different from mine situation like mine.

1. This character:

1 - Is similar to me / 2 / 3 / 4 / 5 / 6/ 7 - Is different from me

m. This character:

1 - Has social status like mine / 2 / 3 / 4 / 5 / 6 / 7 - Has social status different from mine

n. This character:

1 - Is unlike me / 2/3/4/5/6/7 - Is like me

o. This character:

Has a background different from mine / 2 / 3 / 4 / 5 / 6 / 7 - Has a background similar to mine

B. For the following items, please select the response which best describes yourself. Your responses will not be shared, and you may choose not to answer any question which you are uncomfortable answering. 


\section{ARE WE COOL YET?}

1. Please indicate your age:

2. Please indicate your sex:

Female

Male

Other

3. Please indicate the racial category with which you identify:
African-American/Black
American Indian/Alaskan Native
Asian/Pacific Islander
Caucasian
Hispanic or Latina/o
Indian
Two or more races
Other

4. Please indicate your annual household income level:

Less than $\$ 25,000$

$\$ 25,000-\$ 39,999$

$\$ 40,000-\$ 59,999$

$\$ 60,000-\$ 99,999$

$\$ 100,000$ or greater

Not sure

5. On average, how many hours per day do you spend on the Internet?

6. On average, how many hours per week do you spend on the Internet?

7. Do you watch television?

Yes

No

8. If so, what are your top three favorite shows?

9. On average, how many hours of television do you watch per day?

10. On average, how many hours of television do you watch per week? 


\section{ARE WE COOL YET?}

\section{Appendix C: Research Questions and Hypotheses}

RQ1a: How have nerd portrayals in popular television changed in frequency in the past twenty years? (p. 22)

RQ1b: How have nerd portrayals in popular television changed in social competence in the past twenty years? (p. 22)

RQ1c: How have nerd portrayals in popular television changed in physical attractiveness in the past twenty years? (p. 22)

RQ2a: How do individuals currently perceive nerds in popular television? (p. 33)

RQ2b: What are audience perceptions regarding the social competence portrayed by nerd characters in current popular television programs? (p. 33)

RQ2c: What are audience perceptions regarding the physical attractiveness portrayed by nerd characters in current popular television programs? (p. 33)

RQ3: Does the level of social competence displayed by nerd characters in popular television programs vary by sex? (p. 39)

H1: The frequency of portrayals of nerds in popular television programs will vary by sex, with male portrayals being more frequent than female portrayals. (p. 39)

H2: The level of physical attractiveness displayed by nerd characters in popular television programs will vary by sex, with female characters being more physically attractive than male characters. (p. 39) 